\title{
INERTIA AND RANK CHARACTERIZATIONS OF SOME MATRIX EXPRESSIONS*
}

\author{
DELIN CHU ${ }^{\dagger}$, Y. S. HUNG ${ }^{\ddagger}$, AND HUGO J. WOERDEMAN $\S$
}

\begin{abstract}
In this paper we consider the admissible inertias and ranks of the expressions $A-$ $B X B^{*}-C Y C^{*}$ and $A-B X C^{*} \pm C X^{*} B^{*}$ with unknowns $X$ and $Y$ in the four cases when these expressions are: (i) complex self-adjoint, (ii) complex skew-adjoint, (iii) real symmetric, (iv) real skew symmetric. We also provide a construction for $X$ and $Y$ to achieve the desired inertia/rank that uses only unitary/orthogonal transformation, thus leading to a numerically reliable construction. In addition, we look at related block matrix completion problems $\left[\begin{array}{ccc}\mathcal{A} & \mathcal{B} & \mathcal{C} \\ \pm \mathcal{B}^{\star} & \mathcal{X} & \mathcal{E} \\ \pm \mathcal{C}^{\star} & \pm \mathcal{E}^{\star} & \mathcal{Y}\end{array}\right]$ with either two diagonal unknown blocks and $\left[\begin{array}{ccc}\mathcal{A} & \mathcal{B} & \mathcal{X} \\ \pm \mathcal{B}^{\star} & \mathcal{D} & \mathcal{C} \\ \pm \mathcal{X}^{\star} & \pm \mathcal{C}^{\star} & \mathcal{E}\end{array}\right]$ with an unknown off-diagonal block. Finally, we also provide all admissible ranks in the case when we drop any adjointness/symmetry constraint.
\end{abstract}

Key words. rank, inertia, partial matrix, completion, matrix equation

AMS subject classifications. Primary, 15A24; Secondary, 15A18, 65F30

DOI. $10.1137 / 080712945$

1. Introduction. Throughout this paper the following notation will be used:

- $\mathbb{F}=\mathbb{C}$ (complex number field) or $\mathbb{F}=\mathbb{R}$ (real number field);

- For a self-adjoint/symmetric matrix $A=A^{\star} \in \mathbb{F}^{n \times n}$, its inertia

$$
\left.\mathbb{I} n(A)=\left(\mathcal{I}_{+}(A), \mathcal{I}_{-}(A), n-\mathcal{I}_{+}(A)-\mathcal{I}_{-} A\right)\right)
$$

is the triple consisting of the number of positive, negative, and zero eigenvalues of $A$, counting multiplicity;

- For a skew-adjoint/skew-symmetric matrix $A=-A^{\star} \in \mathbb{F}^{n \times n}$, its inertia

$$
\mathbb{I} n(A)=\left(\mathcal{I}_{+}(A), \mathcal{I}_{-}(A), n-\mathcal{I}_{+}(A)-\mathcal{I}_{-}(A)\right)
$$

is the triple consisting of the number of eigenvalues with positive, negative, and zero imaginary part, again counting multiplicity. As $A=-A^{\star}$ implies that $-i A$ is Hermitian, we have that the eigenvalues of $A$ are purely imaginary, and $\mathbb{I} n(A)=\mathbb{I} n(-i A)$, where the latter refers to the inertia of a Hermitian matrix.

In matrix theory and applications, many problems are closely related to the ranks and inertias of some matrix expressions with variable entries, and so it is necessary to explicitly characterize the possible ranks and inertias of the matrix expressions concerned. The study on the possible ranks and inertias of matrix expressions can be traced back to the late 1980s [1, 11, 13]. Recently, the extremal ranks of some matrix expressions have found many applications in control theory [4, 5], statistics,

\footnotetext{
* Received by the editors January 10, 2008; accepted for publication (in revised form) by M. Van Barel March 23, 2009; published electronically October 29, 2009.

http://www.siam.org/journals/simax/31-3/71294.html

$\dagger$ Department of Mathematics, National University of Singapore, 2 Science Drive 2, Singapore 117543 Singapore (matchudl@nus.edu.sg).

${ }^{\ddagger}$ Department of Electrical and Electronic Engineering, The University of Hong Kong, Pokfulam Road, Hong Kong (yshung@hkueee.hku.hk).

$\S$ Department of Mathematics, Drexel University, Philadelphia, PA 19104 (hugo@math.drexel. edu). This author's research was partially supported by NSF grant DMS-0500678.
} 
and economics $[14,15,20,22]$, and hence this topic has been revisited in $[2,6,7,8$, $10,12,16,17,19,21,23,24,25,26]$.

In this paper we will study the admissible ranks and inertias of matrix expressions of the forms

$$
\mathcal{M}:=\left[\begin{array}{ccc}
\mathcal{A} & \mathcal{B} & \mathcal{C} \\
\pm \mathcal{B}^{\star} & \mathcal{X} & \mathcal{E} \\
\pm \mathcal{C}^{\star} & \pm \mathcal{E}^{\star} & \mathcal{Y}
\end{array}\right]
$$

or

$$
\mathcal{M}:=A-B X B^{\star}-C Y C^{\star},
$$

or

$$
\mathcal{M}:=A-B X C^{\star} \pm C X^{\star} B^{\star},
$$

or

$$
\mathcal{M}:=\left[\begin{array}{ccc}
\mathcal{A} & \mathcal{B} & \mathcal{X} \\
\pm \mathcal{B}^{\star} & \mathcal{D} & \mathcal{C} \\
\pm \mathcal{X}^{\star} & \pm \mathcal{C}^{\star} & \mathcal{E}
\end{array}\right]
$$

where the matrices have elements in the field $\mathbb{F}$ and where $X, Y, \mathcal{X}, \mathcal{Y}$ are the unknowns. Whenever we write \pm in a statement, we are really making two statements: one in which one should take all \pm to be + , and one in which one should take all \pm to be - . We consider both the case of complex matrices and real matrices, and the completion will be required to have the same structure as the given data. The possible structures for $\mathcal{M}$ we consider are

(i) self-adjoint: $\mathbb{F}=\mathbb{C}$ and $\mathcal{M}=\mathcal{M}^{*}$,

(ii) skew-adjoint: $\mathbb{F}=\mathbb{C}$ and $\mathcal{M}=-\mathcal{M}^{*}$,

(iii) symmetric: $\mathbb{F}=\mathbb{R}$ and $\mathcal{M}=\mathcal{M}^{*}=\mathcal{M}^{T}$,

(iv) skew-symmetric: $\mathbb{F}=\mathbb{R}$ and $\mathcal{M}=-\mathcal{M}^{*}=-\mathcal{M}^{T}$.

In cases (i) and (iii) we are interested in the rank and numbers of positive and negative eigenvalues $\left(\mathcal{I}_{ \pm}(\mathcal{M})\right)$ of a completion of $\mathcal{M}$, while in cases (ii) and (iv) we are interested in the rank and numbers of eigenvalues with positive and negative imaginary part (also denoted by $\mathcal{I}_{ \pm}(\mathcal{M})$ ). Note that when $\mathcal{M}=-\mathcal{M}^{T} \in \mathbb{R}^{N \times N}$, we have that $\mathcal{I}_{+}(\mathcal{M})=\mathcal{I}_{-}(\mathcal{M})$ as the eigenvalues of a real matrix appear in conjugate pairs. As a consequence, we have that the rank of a real skew-symmetric matrix is always even. We will see that the skew-symmetric case distinguishes itself from the other cases because of these observations.

Our first result concerns the expression $A-B X B^{*}-C Y C^{*}$.

Theorem 1. Let $A= \pm A^{\star} \in \mathbb{F}^{n \times n}, B \in \mathbb{F}^{n \times m}$, and $C \in \mathbb{F}^{n \times p}$. Denote

$$
\begin{aligned}
k_{\min }= & * 2 \operatorname{rank}\left[\begin{array}{ccc}
A & B & C
\end{array}\right]+\operatorname{rank}\left[\begin{array}{cc}
A & B \\
C^{\star} & 0
\end{array}\right]-\operatorname{rank}\left[\begin{array}{ccc}
A & B & C \\
B^{\star} & 0 & 0
\end{array}\right] \\
& -\operatorname{rank}\left[\begin{array}{ccc}
A & B & C \\
C^{\star} & 0 & 0
\end{array}\right] .
\end{aligned}
$$

(a) If $\mathbb{F}=\mathbb{C}$, then

$$
\begin{gathered}
\left\{\operatorname{rank}\left(A-B X B^{\star}-C Y C^{\star}\right) \mid X= \pm X^{\star} \in \mathbb{F}^{m \times m}, Y= \pm Y^{\star} \in \mathbb{F}^{p \times p}\right\} \\
=\left\{s \mid s \text { is integer, } k_{\min } \leq s \leq \operatorname{rank}\left[\begin{array}{ccc}
A & B & C
\end{array}\right]\right\},
\end{gathered}
$$

Copyright (c) by SIAM. Unauthorized reproduction of this article is prohibited. 
and for any given integers $\mathcal{I}_{+}$and $\mathcal{I}_{-}$, there exist matrices $X= \pm X^{\star} \in \mathbb{F}^{m \times m}$ and $Y= \pm Y^{\star} \in \mathbb{F}^{p \times p}$ such that

$$
\mathbb{I n}\left(A-B X B^{\star}-C Y C^{\star}\right)=\left(\mathcal{I}_{+}, \mathcal{I}_{-}, n-\mathcal{I}_{+}-\mathcal{I}_{-}\right)
$$

if and only if

(6)

$$
\left\{\begin{array}{l}
\operatorname{rank}\left[\begin{array}{lll}
A & B & C
\end{array}\right]+\mathcal{I}_{+}\left(\left[\begin{array}{ccc}
A & B & C \\
B^{\star} & 0 & 0 \\
C^{\star} & 0 & 0
\end{array}\right]\right)-\operatorname{rank}\left[\begin{array}{ccc}
A & B & C \\
B^{\star} & 0 & 0 \\
C^{\star} & 0 & 0
\end{array}\right] \leq \mathcal{I}_{+}, \\
\operatorname{rank}\left[\begin{array}{lll}
A & B & C
\end{array}\right]+\mathcal{I}_{-}\left(\left[\begin{array}{ccc}
A & B & C \\
B^{\star} & 0 & 0 \\
C^{\star} & 0 & 0
\end{array}\right]\right)-\operatorname{rank}\left[\begin{array}{ccc}
A & B & C \\
B^{\star} & 0 & 0 \\
C^{\star} & 0 & 0
\end{array}\right] \leq \mathcal{I}_{-}, \\
k_{\min } \leq \mathcal{I}_{+}+\mathcal{I}_{-} \leq \operatorname{rank}\left[\begin{array}{ccc}
A & C
\end{array}\right] .
\end{array}\right.
$$

(b)

- If $\mathbb{F}=\mathbb{R}$ and $A=A^{T}$, then

$$
\begin{gathered}
\left\{\operatorname{rank}\left(A-B X B^{T}-C Y C^{T}\right) \mid X=X^{T} \in \mathbb{R}^{m \times m}, Y=Y^{T} \in \mathbb{R}^{p \times p}\right\} \\
=\left\{s \mid s \text { is integer, } k_{\min } \leq s \leq \operatorname{rank}\left[\begin{array}{ccc}
A & B & C
\end{array}\right]\right\},
\end{gathered}
$$

and for any given integers $\mathcal{I}_{+}$and $\mathcal{I}_{-}$, there exist matrices $X=X^{T} \in \mathbb{R}^{m \times m}$ and $Y=Y^{T} \in \mathbb{R}^{p \times p}$ such that

$$
\mathbb{I} n\left(A-B X B^{T}-C Y C^{T}\right)=\left(\mathcal{I}_{+}, \mathcal{I}_{-}, n-\mathcal{I}_{+}-\mathcal{I}_{-}\right)
$$

if and only if (6) holds.

- If $\mathbb{F}=\mathbb{R}$ and $A=-A^{T}$,

(i) when

$$
\begin{aligned}
\operatorname{rank}\left[\begin{array}{ccc}
A & B & C \\
B^{T} & 0 & 0
\end{array}\right]+\operatorname{rank}\left[\begin{array}{ccc}
A & B & C \\
C^{T} & 0 & 0
\end{array}\right]= & \operatorname{rank}\left[\begin{array}{ccc}
A & B & C
\end{array}\right] \\
& +\operatorname{rank}\left[\begin{array}{ccc}
A & B & C \\
B^{T} & 0 & 0 \\
C^{T} & 0 & 0
\end{array}\right], \\
\operatorname{rank}\left[\begin{array}{ccc}
A & B & C
\end{array}\right]= & \operatorname{rank}\left[\begin{array}{cc}
A & C \\
B^{T} & 0
\end{array}\right],
\end{aligned}
$$

and

$$
\begin{aligned}
& \operatorname{rank}\left[\begin{array}{ccc}
A & B & C \\
B^{T} & 0 & 0
\end{array}\right]-\operatorname{rank}\left[\begin{array}{cc}
A & C \\
B^{T} & 0
\end{array}\right], \\
& \operatorname{rank}\left[\begin{array}{ccc}
A & B & C \\
C^{T} & 0 & 0
\end{array}\right]-\operatorname{rank}\left[\begin{array}{cc}
A & C \\
B^{T} & 0
\end{array}\right]
\end{aligned}
$$

are both odd, then

$\left\{\operatorname{rank}\left(A-B X B^{T}-C Y C^{T}\right) \mid X=-X^{T} \in \mathbb{R}^{m \times m}, Y=-Y^{T} \in \mathbb{R}^{p \times p}\right\}$

(8) $=\left\{s \mid s\right.$ is even integer, $\left.k_{\min } \leq s \leq \operatorname{rank}\left[\begin{array}{lll}A & B & C\end{array}\right]-2\right\}$,

Copyright $@$ by SIAM. Unauthorized reproduction of this article is prohibited. 
and for any given integers $\mathcal{I}_{+}$and $\mathcal{I}_{-}$, there exist matrices $X=-X^{T} \in$ $\mathbb{R}^{m \times m}$ and $Y=-Y^{T} \in \mathbb{R}^{p \times p}$ such that

$$
\operatorname{In}\left(A-B X B^{T}-C Y C^{T}\right)=\left(\mathcal{I}_{+}, \mathcal{I}_{-}, n-\mathcal{I}_{+}-\mathcal{I}_{-}\right)
$$

if and only if

$$
\mathcal{I}_{+}=\mathcal{I}_{-}, \quad k_{\min } \leq 2 \mathcal{I}_{+} \leq \operatorname{rank}\left[\begin{array}{ccc}
A & B & C
\end{array}\right]-2
$$

(ii) otherwise,

$$
\begin{gathered}
\left\{\operatorname{rank}\left(A-B X B^{T}-C Y C^{T}\right) \mid X=-X^{T} \in \mathbb{R}^{m \times m}, Y=-Y^{T} \in \mathbb{R}^{p \times p}\right\} \\
(10)=\left\{s \mid s \text { is even integer, } k_{\min } \leq s \leq \operatorname{rank}\left[\begin{array}{ccc}
A & B & C
\end{array}\right]\right\},
\end{gathered}
$$

and for any given integers $\mathcal{I}_{+}$and $\mathcal{I}_{-}$, there exist matrices $X=-X^{T} \in$ $\mathbb{R}^{m \times m}$ and $Y=-Y^{T} \in \mathbb{R}^{p \times p}$ such that

$$
\mathbb{I} n\left(A-B X B^{T}-C Y C^{T}\right)=\left(\mathcal{I}_{+}, \mathcal{I}_{-}, n-\mathcal{I}_{+}-\mathcal{I}_{-}\right)
$$

if and only if

$$
\mathcal{I}_{+}=\mathcal{I}_{-}, \quad k_{\min } \leq 2 \mathcal{I}_{+} \leq \operatorname{rank}\left[\begin{array}{ccc}
A & B & C
\end{array}\right]
$$

The above theorem settles a conjecture proposed in [21, Conjecture 2.7] regarding the maximal and minimal ranks of $A-B X B^{\star}-C Y C^{\star}$ in the case of $\mathbb{F}=\mathbb{C}$. The authors correctly identified the minimal and maximal admissible ranks for the selfadjoint/skew-adjoint cases. It must be highlighted, however, that the ranges of the admissible ranks of the expression $A-B X B^{T}-C Y C^{T}$ are substantially different in the real skew-symmetric case. Of course, as we observed before, the rank is only allowed to be even. But that is not the full story, as the following example shows.

Example 1. Let

$$
A=\left[\begin{array}{ll}
0 & 0 \\
0 & 0
\end{array}\right], \quad B=\left[\begin{array}{ll}
1 & 0 \\
0 & 0
\end{array}\right], \quad C=\left[\begin{array}{ll}
0 & 0 \\
0 & 1
\end{array}\right]
$$

Then, letting $X=-X^{T}, Y=-Y^{T} \in \mathbb{R}^{2 \times 2}$, we get that $A-B X B^{T}-C X C^{T}$ must equal 0, and thus the maximal rank of this expression is rank $\left[\begin{array}{ccc}A & B & C\end{array}\right]-2$. If we consider the complex analogue, we can indeed achieve rank $\left[\begin{array}{lll}A & B & C\end{array}\right]$ by choosing, for instance, $X=Y=i I_{2}$.

The next corollary is a direct application of Theorem 1.

Corollary 2. Let $A= \pm A^{\star} \in \mathbb{F}^{n \times n}, B \in \mathbb{F}^{n \times m}$, and $C \in \mathbb{F}^{n \times p}$. Then the matrix equation

$$
A-B X B^{\star}-C Y C^{\star}=0
$$

is solvable, with $X= \pm X^{\star} \in \mathbb{F}^{m \times m}$ and $Y= \pm Y^{\star} \in \mathbb{F}^{p \times p}$ if and only if

$2 \operatorname{rank}\left[\begin{array}{ccc}A & B & C\end{array}\right]+\operatorname{rank}\left[\begin{array}{cc}A & B \\ C^{\star} & 0\end{array}\right]=\operatorname{rank}\left[\begin{array}{ccc}A & B & C \\ B^{\star} & 0 & 0\end{array}\right]+\operatorname{rank}\left[\begin{array}{ccc}A & B & C \\ C^{\star} & 0 & 0\end{array}\right]$.

Next we address the expression $A-B X C^{*} \pm C X^{*} B^{*}$. 
Theorem 3. Let $A= \pm A^{*} \in \mathbb{F}^{n \times n}, B \in \mathbb{F}^{n \times m}$, and $C \in \mathbb{F}^{n \times p}$. Denote

$$
\begin{aligned}
k_{\min }= & \max \left\{\mathcal{I}_{+}\left(\left[\begin{array}{cc}
A & B \\
\pm B^{*} & 0
\end{array}\right]\right)+\operatorname{rank}\left[\begin{array}{ccc}
A & B & C
\end{array}\right]-\operatorname{rank}\left[\begin{array}{ccc}
A & B & C \\
B^{*} & 0 & 0
\end{array}\right],\right. \\
& \left.\mathcal{I}_{+}\left(\left[\begin{array}{cc}
A & C \\
\pm C^{*} & 0
\end{array}\right]\right)+\operatorname{rank}\left[\begin{array}{ccc}
A & B & C
\end{array}\right]-\operatorname{rank}\left[\begin{array}{ccc}
A & B & C \\
C^{*} & 0 & 0
\end{array}\right]\right\} \\
& +\max \left\{\mathcal{I}_{-}\left(\left[\begin{array}{cc}
A & B \\
\pm B^{*} & 0
\end{array}\right]\right)+\operatorname{rank}\left[\begin{array}{lll}
A & B & C
\end{array}\right]-\operatorname{rank}\left[\begin{array}{ccc}
A & B & C \\
B^{*} & 0 & 0
\end{array}\right],\right. \\
& \left.\mathcal{I}_{-}\left(\left[\begin{array}{cc}
A & C \\
\pm C^{*} & 0
\end{array}\right]\right)+\operatorname{rank}\left[\begin{array}{lll}
A & B & C
\end{array}\right]-\operatorname{rank}\left[\begin{array}{ccc}
A & B & C \\
C^{*} & 0 & 0
\end{array}\right]\right\}
\end{aligned}
$$

and

$$
k_{\max }=\min \left\{\operatorname{rank}\left[\begin{array}{cc}
A & B \\
B^{*} & 0
\end{array}\right], \operatorname{rank}\left[\begin{array}{cc}
A & C \\
C^{*} & 0
\end{array}\right], \operatorname{rank}\left[\begin{array}{ccc}
A & B & C
\end{array}\right]\right\} .
$$

(a) If $\mathbb{F}=\mathbb{C}$, then

$$
\begin{aligned}
& \left\{\operatorname{rank}\left(A-B X C^{\star} \pm C X^{\star} B^{\star}\right) \mid X=\mathbb{F}^{m \times p}\right\} \\
& \quad=\left\{s \mid s \text { is integer and } k_{\min } \leq s \leq k_{\max }\right\},
\end{aligned}
$$

and for any two integers $\mathcal{I}_{+}$and $\mathcal{I}_{-}$, there exists an $X \in \mathbb{F}^{m \times p}$ such that

$$
\mathbb{I} n\left(A-B X C^{*} \pm C X^{*} B^{*}\right)=\left(\mathcal{I}_{+}, \mathcal{I}_{-}, n-\mathcal{I}_{+}-\mathcal{I}_{-}\right)
$$

if and only if

$$
\left\{\begin{array}{c}
\mathcal{I}_{+} \geq \max \left\{\mathcal{I}_{+}\left(\left[\begin{array}{cc}
A & B \\
\pm B^{*} & 0
\end{array}\right]\right)+\operatorname{rank}\left[\begin{array}{ccc}
A & B & C
\end{array}\right]-\operatorname{rank}\left[\begin{array}{ccc}
A & B & C \\
B^{*} & 0 & 0
\end{array}\right]\right. \\
\left.\mathcal{I}_{+}\left(\left[\begin{array}{cc}
A & C \\
\pm C^{*} & 0
\end{array}\right]\right)+\operatorname{rank}\left[\begin{array}{ccc}
A & B & C
\end{array}\right]-\operatorname{rank}\left[\begin{array}{ccc}
A & B & C \\
C^{*} & 0 & 0
\end{array}\right]\right\} \\
\mathcal{I}_{-} \geq \max \left\{\mathcal{I}_{-}\left(\left[\begin{array}{cc}
A & B \\
\pm B^{*} & 0
\end{array}\right]\right)+\operatorname{rank}\left[\begin{array}{ccc}
A & B & C
\end{array}\right]-\operatorname{rank}\left[\begin{array}{ccc}
A & B & C \\
B^{*} & 0 & 0
\end{array}\right]\right. \\
\left.\mathcal{I}_{-}\left(\left[\begin{array}{cc}
A & C \\
\pm C^{*} & 0
\end{array}\right]\right)+\operatorname{rank}\left[\begin{array}{ccc}
A & B & C
\end{array}\right]-\operatorname{rank}\left[\begin{array}{ccc}
A & B & C \\
C^{*} & 0 & 0
\end{array}\right]\right\} \\
\mathcal{I}_{+} \leq \min \left\{\mathcal{I}_{+}\left(\left[\begin{array}{cc}
A & B \\
\pm B^{*} & 0
\end{array}\right]\right), \mathcal{I}_{+}\left(\left[\begin{array}{cc}
A & C \\
\pm C^{*} & 0
\end{array}\right]\right)\right\}, \\
\mathcal{I}_{-} \leq \min \left\{\mathcal{I}_{-}\left(\left[\begin{array}{cc}
A & B \\
\pm B^{*} & 0
\end{array}\right]\right), \mathcal{I}_{-}\left(\left[\begin{array}{cc}
A & C \\
\pm C^{*} & 0
\end{array}\right]\right)\right\} \\
\mathcal{I}_{+}-\mathcal{I}_{-} \geq-\mathcal{I}_{-}\left(\left[\begin{array}{cc}
A & B \\
\pm B^{*} & 0
\end{array}\right]\right)-\mathcal{I}_{-}\left(\left[\begin{array}{cc}
A & C \\
\pm C^{*} & 0
\end{array}\right]\right)+\operatorname{rank}\left[\begin{array}{ccc}
A & B & C
\end{array}\right] \\
\mathcal{I}_{+}-\mathcal{I}_{-} \leq \mathcal{I}_{+}\left(\left[\begin{array}{cc}
A & B \\
\pm B^{*} & 0
\end{array}\right]\right)+\mathcal{I}_{+}\left(\left[\begin{array}{cc}
A & C \\
\pm C^{*} & 0
\end{array}\right]\right)-\operatorname{rank}\left[\begin{array}{lll}
A & B & C
\end{array}\right] \\
\mathcal{I}_{-}+\mathcal{I}_{+} \leq \operatorname{rank}\left[\begin{array}{ccc}
A & B & C
\end{array}\right] ;
\end{array}\right.
$$


(b) If $\mathbb{F}=\mathbb{R}$ and $A=A^{T}$, then

$$
\begin{array}{r}
\left\{\operatorname{rank}\left(A-B X C^{T}-C X^{T} B^{T}\right) \mid X=\mathbb{R}^{m \times p}\right\} \\
=\left\{s \mid s \text { is integer and } k_{\min } \leq s \leq k_{\max }\right\},
\end{array}
$$

and for any two integers $\mathcal{I}_{+}$and $\mathcal{I}_{-}$, there exists an $X \in \mathbb{R}^{m \times p}$ such that

$$
\mathbb{I} n\left(A-B X C^{T}-C X^{T} B^{T}\right)=\left(\mathcal{I}_{+}, \mathcal{I}_{-}, n-\mathcal{I}_{+}-\mathcal{I}_{-}\right)
$$

if and only (13) holds;

(c) If $\mathbb{F}=\mathbb{R}$ and $A=-A^{T}$, then $k_{\min }$ is reduced to

$$
\begin{aligned}
k_{\min }=\max & \left\{\operatorname{rank}\left[\begin{array}{cc}
A & B \\
B^{T} & 0
\end{array}\right]+2\left(\operatorname{rank}\left[\begin{array}{lll}
A & B & C
\end{array}\right]-\operatorname{rank}\left[\begin{array}{ccc}
A & B & C \\
B^{T} & 0 & 0
\end{array}\right]\right),\right. \\
& \left.\operatorname{rank}\left[\begin{array}{cc}
A & C \\
C^{T} & 0
\end{array}\right]+2\left(\operatorname{rank}\left[\begin{array}{lll}
A & B & C
\end{array}\right]-\operatorname{rank}\left[\begin{array}{ccc}
A & B & C \\
C^{T} & 0 & 0
\end{array}\right]\right)\right\},
\end{aligned}
$$

and

$$
\begin{aligned}
& \left\{\operatorname{rank}\left(A-B X C^{T}+C X^{T} B^{T}\right) \mid X \in \mathbb{R}^{m \times p}\right\} \\
& \quad=\left\{s \mid s \text { is even integer and } k_{\min } \leq s \leq k_{\max }\right\}
\end{aligned}
$$

furthermore, for any two integers $\mathcal{I}_{+}$and $\mathcal{I}_{-}$, there exists an $X \in \mathbb{F}^{m \times p}$ such that

$$
\operatorname{In}\left(A-B X C^{T}+C X^{T} B^{T}\right)=\left(\mathcal{I}_{+}, \mathcal{I}_{-}, n-\mathcal{I}_{+}-\mathcal{I}_{-}\right)
$$

if and only

$$
\mathcal{I}_{+}=\mathcal{I}_{-}, \quad k_{\min } \leq 2 \mathcal{I}_{+} \leq k_{\max } .
$$

The above theorem settles another conjecture proposed in [21] regarding the maximal and minimal ranks of $A-B X C^{\star} \pm C X^{\star} B^{\star}$ in the case of $\mathbb{F}=\mathbb{C}$. The authors correctly identified the maximal possible rank but incorrectly identified the minimal possible rank. Indeed, they suggested the following quantity for the minimal rank:

$$
\begin{aligned}
k=\max & \left\{\operatorname{rank}\left[\begin{array}{cc}
A & B \\
\pm B^{*} & 0
\end{array}\right]+2 \operatorname{rank}\left[\begin{array}{lll}
A & B & C
\end{array}\right]-2 \operatorname{rank}\left[\begin{array}{ccc}
A & B & C \\
B^{*} & 0 & 0
\end{array}\right]\right. \\
& \left.\operatorname{rank}\left[\begin{array}{cc}
A & C \\
\pm C^{*} & 0
\end{array}\right]+2 \operatorname{rank}\left[\begin{array}{lll}
A & B & C
\end{array}\right]-2 \operatorname{rank}\left[\begin{array}{ccc}
A & B & C \\
C^{*} & 0 & 0
\end{array}\right]\right\}
\end{aligned}
$$

The following example shows the difference between this guess and $k_{\min }$ from Theorem 3 .

Example 2. Let

$$
A=\left[\begin{array}{cc}
1 & 0 \\
0 & -1
\end{array}\right], \quad B=\left[\begin{array}{l}
1 \\
0
\end{array}\right], \quad C=\left[\begin{array}{l}
0 \\
1
\end{array}\right]
$$

Then, letting $X \in \mathbb{C}$, we get that $A-B X C^{*}-C X^{*} B^{*}=\left[\begin{array}{cc}1 & -X \\ -X^{\star} & -1\end{array}\right]$ has minimal rank 2 (as the determinant is $-1-|X|^{2} \neq 0$ for all $X \in \mathbb{C}$ ). Indeed, in this case we find that $k_{\min }$ in Theorem 3 equals 2 . However, the quantity $k$ above equals 1 . For an example in the skew-adjoint case, one can consider $i A-B X C^{*}+C X^{*} B^{*}=$ $\left[\begin{array}{cc}i & -X \\ X^{\star} & -i\end{array}\right]$ which also has minimal rank 2.

Copyright $@$ by SIAM. Unauthorized reproduction of this article is prohibited. 
The possible ranks in Theorem 3 for the case when $C=I$ appeared recently in [12]. The next corollary is a trivial consequence of Theorem 3.

Corollary 4. Let $A= \pm A^{*} \in \mathbb{F}^{n \times n}, B \in \mathbb{F}^{n \times m}$, and $C \in \mathbb{F}^{n \times p}$. Let $k_{\min }$ be defined in Theorem 3. Then there exists an $X \in \mathbb{F}^{m \times p}$ such that

$$
A-B X C^{\star} \pm C X^{\star} B^{\star}=0
$$

if and only if

$$
k_{\min }=0 .
$$

The paper is organized as follows. In section 2 we present our result regarding the admissible ranks and inertias of the matrix $\mathcal{M}$ of the form (1). Subsequently, we will prove Theorem 1 as a corollary. In section 3, we will provide an alternative proof of Theorem 1 that uses only unitary/orthogonal transformations. The importance of this alternative proof is threefold: (i) it provides a numerically reliable way to construct parameter matrices $X$ and $Y$ yielding the desired rank (or inertia) of $A-$ $B X B^{\star}-C Y C^{\star}$ for all cases with $\mathbb{F}=\mathbb{C}$ or $\mathbb{F}=\mathbb{R}$ and $A= \pm A^{\star}$; (ii) such a proof can also be applied to the completions of the partial matrix $\mathcal{M}$ of the form (1), since

$$
\begin{aligned}
& {\left[\begin{array}{ccc}
\mathcal{A} & \mathcal{B} & \mathcal{C} \\
\pm \mathcal{B}^{\star} & \mathcal{X} & \mathcal{E} \\
\pm \mathcal{C}^{\star} & \pm \mathcal{E}^{\star} & \mathcal{Y}
\end{array}\right] }=\left[\begin{array}{ccc}
\mathcal{A} & \mathcal{B} & \mathcal{C} \\
\pm \mathcal{B}^{\star} & 0 & \mathcal{E} \\
\pm \mathcal{C}^{\star} & \pm \mathcal{E}^{\star} & 0
\end{array}\right]-\left[\begin{array}{l}
0 \\
I \\
0
\end{array}\right](-\mathcal{X})\left[\begin{array}{l}
0 \\
I \\
0
\end{array}\right]^{\star}-\left[\begin{array}{l}
0 \\
0 \\
I
\end{array}\right](-\mathcal{Y})\left[\begin{array}{l}
0 \\
0 \\
I
\end{array}\right]^{\star} \\
&:=A-B X B^{\star}-C Y C^{\star}
\end{aligned}
$$

(iii) its main building components will be used in the proof of Theorem 3. Consequently, a numerically reliable method for constructing the parameter matrix $X$ to achieve any desired rank is embedded in the proof of Theorem 3. While it is essential theoretically to determine the range of achievable ranks and inertia, the numerically reliable computation is equally important in applications. In section 4 we prove Theorem 3. Finally, in section 5 we remove the (skew) adjoint/symmetry conditions to characterize all admissible rank of the partial matrix $\left[\begin{array}{ccc}\mathcal{A} & \mathcal{B} & \mathcal{C} \\ \mathcal{D} & ? & \mathcal{E} \\ \mathcal{F} & \mathcal{G} & ?\end{array}\right]$ and the expression $A-B X C-D Y E$.

2. Ranks and inertias of partial matrices with two unknown block diagonal entries. We study the rank and inertia of the partial matrix $\mathcal{M}$ of the form (1) in this section. Our main result is the following.

Theorem 5. Let $\mathcal{A}= \pm \mathcal{A}^{\star} \in \mathbb{F}^{n \times n}, \mathcal{B} \in \mathbb{F}^{n \times m}, \mathcal{C} \in \mathbb{F}^{n \times p}$, and $\mathcal{E} \in \mathbb{F}^{m \times p}$. Denote

$\mathcal{K}_{\text {min }}=2 \operatorname{rank}\left[\begin{array}{lll}\mathcal{A} & \mathcal{B} & \mathcal{C}\end{array}\right]+\operatorname{rank}\left[\begin{array}{cc}\mathcal{A} & \mathcal{C} \\ \pm \mathcal{B}^{\star} & \mathcal{E}\end{array}\right]-\operatorname{rank}\left[\begin{array}{cc}\mathcal{A} & \mathcal{B}\end{array}\right]-\operatorname{rank}\left[\begin{array}{ll}\mathcal{A} & \mathcal{C}\end{array}\right]$

(a) If $\mathbb{F}=\mathbb{C}$, then

$$
\begin{gathered}
\left\{\operatorname{rank}\left[\begin{array}{ccc}
\mathcal{A} & \mathcal{B} & \mathcal{C} \\
\pm \mathcal{B}^{\star} & \mathcal{X} & \mathcal{E} \\
\pm \mathcal{C}^{\star} & \pm \mathcal{E}^{\star} & \mathcal{Y}
\end{array}\right] \mid \mathcal{X}=\mathcal{X}^{\star} \in \mathbb{F}^{m \times m}, \mathcal{Y}=\mathcal{Y}^{\star} \in \mathbb{F}^{p \times p}\right\} \\
=\left\{s \mid s \text { is integer, } \mathcal{K}_{\min } \leq s \leq \operatorname{rank}\left[\begin{array}{ccc}
\mathcal{A} & \mathcal{B} & \mathcal{C}
\end{array}\right]+m+p\right\}
\end{gathered}
$$

Copyright (c) by SIAM. Unauthorized reproduction of this article is prohibited. 
and for any given integers $\mathcal{I}_{+}$and $\mathcal{I}_{-}$, there exist matrices $\mathcal{X}= \pm \mathcal{X}^{\star} \in \mathbb{F}^{m \times m}$ and $\mathcal{Y}= \pm \mathcal{Y}^{\star} \in \mathbb{F}^{p \times p}$ such that

$$
\operatorname{In}\left(\left[\begin{array}{ccc}
\mathcal{A} & \mathcal{B} & \mathcal{C} \\
\pm \mathcal{B}^{\star} & \mathcal{X} & \mathcal{E} \\
\pm \mathcal{C}^{\star} & \pm \mathcal{E}^{\star} & \mathcal{Y}
\end{array}\right]\right)=\left(\mathcal{I}_{+}, \mathcal{I}_{-}, n+m+p-\mathcal{I}_{+}-\mathcal{I}_{-}\right)
$$

if and only if

$$
\left\{\begin{array}{l}
\mathcal{I}_{+}(\mathcal{A})+\operatorname{rank}\left[\begin{array}{ccc}
\mathcal{A} & \mathcal{B} & \mathcal{C}
\end{array}\right]-\operatorname{rank}(\mathcal{A}) \leq \mathcal{I}_{+} \\
\mathcal{I}_{-}(\mathcal{A})+\operatorname{rank}\left[\begin{array}{lll}
\mathcal{A} & \mathcal{B} & \mathcal{C}
\end{array}\right]-\operatorname{rank}(\mathcal{A}) \leq \mathcal{I}_{-} \\
\mathcal{K}_{\text {min }} \leq \mathcal{I}_{+}+\mathcal{I}_{-} \leq \operatorname{rank}\left[\begin{array}{lll}
\mathcal{A} & \mathcal{B} & \mathcal{C}
\end{array}\right]+m+p
\end{array}\right.
$$

(b)

- If $\mathbb{F}=\mathbb{R}$ and $\mathcal{A}=\mathcal{A}^{T}$, then

$$
\begin{gathered}
\left\{\operatorname{rank}\left[\begin{array}{ccc}
\mathcal{A} & \mathcal{B} & \mathcal{C} \\
\mathcal{B}^{T} & \mathcal{X} & \mathcal{E} \\
\mathcal{C}^{T} & \mathcal{E}^{T} & \mathcal{Y}
\end{array}\right] \mid \mathcal{X}=\mathcal{X}^{T} \in \mathbb{R}^{m \times m}, \mathcal{Y}=\mathcal{Y}^{T} \in \mathbb{R}^{p \times p}\right\} \\
=\left\{s \mid s \text { is integer, } \mathcal{K}_{\min } \leq s \leq \operatorname{rank}\left[\begin{array}{ccc}
\mathcal{A} & \mathcal{B} & \mathcal{C}
\end{array}\right]+m+p\right\},
\end{gathered}
$$

and for any given integers $\mathcal{I}_{+}$and $\mathcal{I}_{-}$, there exist matrices $\mathcal{X}=\mathcal{X}^{T} \in \mathbb{R}^{m \times m}$ and $\mathcal{Y}=\mathcal{Y}^{T} \in \mathbb{R}^{p \times p}$ such that

$$
\mathbb{I n}\left(\left[\begin{array}{ccc}
\mathcal{A} & \mathcal{B} & \mathcal{C} \\
\mathcal{B}^{T} & \mathcal{X} & \mathcal{E} \\
\mathcal{C}^{T} & \mathcal{E}^{T} & \mathcal{Y}
\end{array}\right]\right)=\left(\mathcal{I}_{+}, \mathcal{I}_{-}, n+m+p-\mathcal{I}_{+}-\mathcal{I}_{-}\right)
$$

if and only if (16) holds;

- If $\mathbb{F}=\mathbb{R}$ and $\mathcal{A}=-\mathcal{A}^{T}$,

(i) when

$$
\left\{\begin{array}{l}
\operatorname{rank}\left[\begin{array}{ll}
\mathcal{A} & \mathcal{B}
\end{array}\right]+\operatorname{rank}\left[\begin{array}{cc}
\mathcal{A} & \mathcal{C}
\end{array}\right]=\operatorname{rank}(\mathcal{A})+\operatorname{rank}\left[\begin{array}{ccc}
\mathcal{A} & \mathcal{B} & \mathcal{C}
\end{array}\right] \\
\operatorname{rank}\left[\begin{array}{lll}
\mathcal{A} & \mathcal{B} & \mathcal{C}
\end{array}\right]=\operatorname{rank}\left[\begin{array}{cc}
\mathcal{A} & \mathcal{C} \\
-\mathcal{B}^{T} & \mathcal{E}
\end{array}\right]
\end{array}\right.
$$

and

$$
\begin{aligned}
& \text { both } m+\operatorname{rank}\left[\begin{array}{ll}
\mathcal{A} & \mathcal{C}
\end{array}\right]-\operatorname{rank}\left[\begin{array}{cc}
\mathcal{A} & \mathcal{C} \\
-\mathcal{B}^{T} & \mathcal{E}
\end{array}\right] \\
& \quad \text { and } p+\operatorname{rank}\left[\begin{array}{ll}
\mathcal{A} & \mathcal{B}
\end{array}\right]-\operatorname{rank}\left[\begin{array}{cc}
\mathcal{A} & \mathcal{C} \\
-\mathcal{B}^{T} & \mathcal{E}
\end{array}\right] \text { are odd }
\end{aligned}
$$

then

$$
\begin{gathered}
\left\{\operatorname{rank}\left[\begin{array}{ccc}
\mathcal{A} & \mathcal{B} & \mathcal{C} \\
-\mathcal{B}^{T} & \mathcal{X} & \mathcal{E} \\
-\mathcal{C}^{T} & -\mathcal{E}^{T} & \mathcal{Y}
\end{array}\right] \mid \mathcal{X}=-\mathcal{X}^{T} \in \mathbb{R}^{m \times m}, \mathcal{Y}=-\mathcal{Y}^{T} \in \mathbb{R}^{p \times p}\right\} \\
\quad=\left\{s \mid s \text { is even integer, } \mathcal{K}_{\text {min }} \leq s \leq \operatorname{rank}\left[\begin{array}{ccc}
\mathcal{A} & \mathcal{B} & \mathcal{C}
\end{array}\right]+m+p-2\right\}
\end{gathered}
$$

Copyright $@$ by SIAM. Unauthorized reproduction of this article is prohibited. 
and for any given integers $\mathcal{I}_{+}$and $\mathcal{I}_{-}$, there exist matrices $\mathcal{X}=-\mathcal{X}^{T} \in$ $\mathbb{R}^{m \times m}$ and $\mathcal{Y}=-\mathcal{Y}^{T} \in \mathbb{R}^{p \times p}$ such that

$$
\operatorname{In}\left(\left[\begin{array}{ccc}
\mathcal{A} & \mathcal{B} & \mathcal{C} \\
-\mathcal{B}^{T} & \mathcal{X} & \mathcal{E} \\
-\mathcal{C}^{T} & -\mathcal{E}^{T} & \mathcal{Y}
\end{array}\right]\right)=\left(\mathcal{I}_{+}, \mathcal{I}_{-}, n+m+p-\mathcal{I}_{+}-\mathcal{I}_{-}\right)
$$

if and only if

$$
\mathcal{I}_{+}=\mathcal{I}_{-}, \quad \mathcal{K}_{\text {min }} \leq 2 \mathcal{I}_{+} \leq \operatorname{rank}\left[\begin{array}{lll}
\mathcal{A} & \mathcal{B} & \mathcal{C}
\end{array}\right]+m+p-2 ;
$$

(ii) otherwise,

$$
\begin{gathered}
\left\{\operatorname{rank}\left[\begin{array}{ccc}
\mathcal{A} & \mathcal{B} & \mathcal{C} \\
-\mathcal{B}^{T} & \mathcal{X} & \mathcal{E} \\
-\mathcal{C}^{T} & -\mathcal{E}^{T} & \mathcal{Y}
\end{array}\right] \mid \mathcal{X}=-\mathcal{X}^{T} \in \mathbb{R}^{m \times m}, \mathcal{Y}=-\mathcal{Y}^{T} \in \mathbb{R}^{p \times p}\right\} \\
\quad=\left\{s \mid s \text { is even integer, } \mathcal{K}_{\min } \leq s \leq \operatorname{rank}\left[\begin{array}{ccc}
\mathcal{A} & \mathcal{B} & \mathcal{C}
\end{array}\right]+m+p\right\},
\end{gathered}
$$

and for any given integers $\mathcal{I}_{+}$and $\mathcal{I}_{-}$, there exist matrices $\mathcal{X}=-\mathcal{X}^{T} \in$ $\mathbb{R}^{m \times m}$ and $\mathcal{Y}=-\mathcal{Y}^{T} \in \mathbb{R}^{p \times p}$ such that

$$
\mathbb{I n}\left(\left[\begin{array}{ccc}
\mathcal{A} & \mathcal{B} & \mathcal{C} \\
-\mathcal{B}^{T} & \mathcal{X} & \mathcal{E} \\
-\mathcal{C}^{T} & -\mathcal{E}^{T} & \mathcal{Y}
\end{array}\right]\right)=\left(\mathcal{I}_{+}, \mathcal{I}_{-}, n+m+p-\mathcal{I}_{+}-\mathcal{I}_{-}\right)
$$

if and only if

$$
\mathcal{I}_{+}=\mathcal{I}_{-}, \quad \mathcal{K}_{\min } \leq 2 \mathcal{I}_{+} \leq \operatorname{rank}\left[\begin{array}{lll}
\mathcal{A} & \mathcal{B} & \mathcal{C}
\end{array}\right]+m+p .
$$

We will need some auxiliary results for the proof of Theorem 5 .

Lemma 6. Let $\mathcal{A}=-\mathcal{A}^{T} \in \mathbb{R}^{n \times n}$. Then $\mathcal{I}_{+}(\mathcal{A})=\mathcal{I}_{-}(\mathcal{A})$, and $\operatorname{rank}(\mathcal{A})=$ $2 \mathcal{I}_{+}(\mathcal{A})$ and thus is an even integer.

Proof. As the characteristic polynomial of $\mathcal{A}$ has real coefficients, its zeroes appear in conjugate pairs. This yields that $\mathcal{I}_{+}(\mathcal{A})=\mathcal{I}_{-}(\mathcal{A})$, and $\operatorname{rank}(\mathcal{A})=2 \mathcal{I}_{+}(\mathcal{A})$.

LEMMA 7. Let

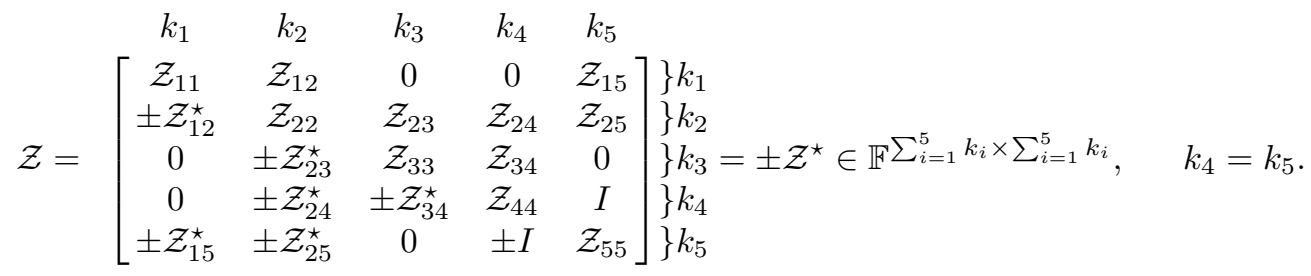

Then,

$\left\{\operatorname{rank}(\mathcal{Z}) \mid \mathcal{Z}= \pm \mathcal{Z}^{\star} \in \mathbb{C}^{\sum_{i=1}^{5} k_{i} \times \sum_{i=1}^{5} k_{i}}\right\}=\left\{s \mid s\right.$ is integer and $\left.k_{5} \leq s \leq \sum_{i=1}^{5} k_{i}\right\}$

and

$$
\begin{aligned}
& \left\{\operatorname{In}(\mathcal{Z}) \mid \mathcal{Z}= \pm \mathcal{Z}^{\star} \in \mathbb{C}^{\sum_{i=1}^{5} k_{i} \times \sum_{i=1}^{5} k_{i}}\right\}=\left\{\left(\mathcal{I}_{+}, \mathcal{I}_{-}, \sum_{i=1}^{5} k_{i}-\mathcal{I}_{+}-\mathcal{I}_{-}\right) \mid \mathcal{I}_{+}\right. \\
& \text {and } \left.\mathcal{I}_{-} \text {are nonnegative integers and } k_{5} \leq \mathcal{I}_{+}+\mathcal{I}_{-} \leq \sum_{i=1}^{5} k_{i}\right\} .
\end{aligned}
$$

Copyright $@$ by SIAM. Unauthorized reproduction of this article is prohibited. 
(ii)

$\left\{\operatorname{rank}(\mathcal{Z}) \mid \mathcal{Z}=\mathcal{Z}^{T} \in \mathbb{R}^{\sum_{i=1}^{5} k_{i} \times \sum_{i=1}^{5} k_{i}}\right\}=\left\{s \mid s\right.$ is integer and $\left.k_{5} \leq s \leq \sum_{i=1}^{5} k_{i}\right\}$,

and

$$
\begin{aligned}
& \left\{\operatorname{In}(\mathcal{Z}) \mid \mathcal{Z}=\mathcal{Z}^{T} \in \mathbb{R}^{\sum_{i=1}^{5} k_{i} \times \sum_{i=1}^{5} k_{i}}\right\}=\left\{\left(\mathcal{I}_{+}, \mathcal{I}_{-}, \sum_{i=1}^{5} k_{i}-\mathcal{I}_{+}-\mathcal{I}_{-}\right) \mid \mathcal{I}_{+}\right. \\
& \text {and } \left.\mathcal{I}_{-} \text {are nonnegative integers and } k_{5} \leq \mathcal{I}_{+}+\mathcal{I}_{-} \leq \sum_{i=1}^{5} k_{i}\right\}
\end{aligned}
$$

(iii) when $k_{2}=0, k_{5}=0, k_{1}$ and $k_{3}$ are odd, then

$$
\begin{aligned}
& \left\{\operatorname{rank}(\mathcal{Z}) \mid \mathcal{Z}=-\mathcal{Z}^{T} \in \mathbb{R}^{\sum_{i=1}^{5} k_{i} \times \sum_{i=1}^{5} k_{i}}\right\} \\
& \quad=\left\{s \mid s \text { is even integer and } k_{5} \leq s \leq \sum_{i=1}^{5} k_{i}-2\right\},
\end{aligned}
$$

and

$$
\begin{aligned}
& \left\{\operatorname{In}(\mathcal{Z}) \mid \mathcal{Z}=-\mathcal{Z}^{T} \in \mathbb{R}^{\sum_{i=1}^{5} k_{i} \times \sum_{i=1}^{5} k_{i}}\right\} \\
& \quad=\left\{\left(\mathcal{I}_{+}, \mathcal{I}_{+}, \sum_{i=1}^{5} k_{i}-2 \mathcal{I}_{+}\right) \mid k_{5} \leq 2 \mathcal{I}_{+} \leq \sum_{i=1}^{5} k_{i}-2\right\},
\end{aligned}
$$

otherwise,

$$
\begin{aligned}
& \left\{\operatorname{rank}(\mathcal{Z}) \mid \mathcal{Z}=-\mathcal{Z}^{T} \in \mathbb{R}^{\sum_{i=1}^{5} k_{i} \times \sum_{i=1}^{5} k_{i}}\right\} \\
& \quad=\left\{s \mid s \text { is even integer and } k_{5} \leq s \leq \sum_{i=1}^{5} k_{i}\right\},
\end{aligned}
$$

and

$$
\begin{aligned}
& \left\{\operatorname{In}(\mathcal{Z}) \mid \mathcal{Z}=-\mathcal{Z}^{T} \in \mathbb{R}^{\sum_{i=1}^{5} k_{i} \times \sum_{i=1}^{5} k_{i}}\right\} \\
& \quad=\left\{\left(\mathcal{I}_{+}, \mathcal{I}_{+}, \sum_{i=1}^{5} k_{i}-2 \mathcal{I}_{+}\right) \mid k_{5} \leq 2 \mathcal{I}_{+} \leq \sum_{i=1}^{5} k_{i}\right\} .
\end{aligned}
$$

Proof. Note that

$$
\begin{aligned}
& \left\{s \mid s \text { is integer and } k_{5} \leq s \leq \sum_{i=1}^{5} k_{i}\right\} \\
\subset & \left\{\operatorname{rank}\left[\begin{array}{ccccc}
\mathcal{Z}_{11} & 0 & 0 & 0 & 0 \\
0 & \mathcal{Z}_{22} & 0 & 0 & 0 \\
0 & 0 & \mathcal{Z}_{33} & 0 & 0 \\
0 & 0 & 0 & \mathcal{Z}_{44} & I \\
0 & 0 & 0 & I & \mathcal{Z}_{55}
\end{array}\right] \mid \mathcal{Z}_{i i}= \pm \mathcal{Z}_{i i}^{\star} \in \mathbb{C}^{k_{i} \times k_{i}}, i=1, \ldots, 5\right\} \\
\subset & \left\{\operatorname{rank}(\mathcal{Z}) \mid \mathcal{Z}= \pm \mathcal{Z}^{\star} \in \mathbb{C}^{\sum_{i=1}^{5} k_{i} \times \sum_{i=1}^{5} k_{i}}\right\} \subset\left\{s \mid s \text { is integer and } k_{5} \leq s \leq \sum_{i=1}^{5} k_{i}\right\},
\end{aligned}
$$




$$
\begin{aligned}
& \left\{\left(\mathcal{I}_{+}, \mathcal{I}_{-}, \sum_{i=1}^{5} k_{i}-\mathcal{I}_{+}-\mathcal{I}_{-}\right) \mid \mathcal{I}_{+}\right. \\
& \text {and } \left.\mathcal{I}_{-} \text {are nonnegative integers and } k_{5} \leq \mathcal{I}_{+}+\mathcal{I}_{-} \leq \sum_{i=1}^{5} k_{i}\right\} \\
& \subset\left\{\operatorname{In}\left(\left[\begin{array}{ccccc}
\mathcal{Z}_{11} & 0 & 0 & 0 & 0 \\
0 & \mathcal{Z}_{22} & 0 & 0 & 0 \\
0 & 0 & \mathcal{Z}_{33} & 0 & 0 \\
0 & 0 & 0 & \mathcal{Z}_{44} & I \\
0 & 0 & 0 & I & \mathcal{Z}_{55}
\end{array}\right]\right) \mid \mathcal{Z}_{i i}= \pm \mathcal{Z}_{i i}^{\star} \in \mathbb{C}^{k_{i} \times k_{i}}, i=1, \ldots, 5\right\} \\
& \subset\left\{\operatorname{In}(\mathcal{Z}) \mid \mathcal{Z}= \pm \mathcal{Z}^{\star} \in \mathbb{C}^{\sum_{i=1}^{5} k_{i} \times \sum_{i=1}^{5} k_{i}}\right\} \\
& \subset\left\{\left(\mathcal{I}_{+}, \mathcal{I}_{-}, \sum_{i=1}^{5} k_{i}-\mathcal{I}_{+}-\mathcal{I}_{-}\right) \mid \mathcal{I}_{+}\right. \\
& \text {and } \left.\mathcal{I}_{-} \text {are nonnegative integers and } k_{5} \leq \mathcal{I}_{+}+\mathcal{I}_{-} \leq \sum_{i=1}^{5} k_{i}\right\} \text {, } \\
& \left\{s \mid s \text { is even integer and } k_{5} \leq s \leq \sum_{i=1}^{5} k_{i}\right\} \\
& =\left\{\operatorname{rank}\left[\begin{array}{ccccc}
\mathcal{Z}_{11} & 0 & 0 & 0 & 0 \\
0 & \mathcal{Z}_{22} & 0 & 0 & 0 \\
0 & 0 & \mathcal{Z}_{33} & 0 & 0 \\
0 & 0 & 0 & \mathcal{Z}_{44} & I \\
0 & 0 & 0 & I & \mathcal{Z}_{55}
\end{array}\right] \mid \mathcal{Z}_{i i}=-\mathcal{Z}_{i i}^{T} \in \mathbb{R}^{k_{i} \times k_{i}}, i=1, \ldots, 5\right\} \\
& \subset\left\{\operatorname{rank}(\mathcal{Z}) \mid \mathcal{Z}=-\mathcal{Z}^{T} \in \mathbb{R}^{\sum_{i=1}^{5} k_{i} \times \sum_{i=1}^{5} k_{i}}\right\} \\
& \subset\left\{s \mid s \text { is even integer and } k_{5} \leq s \leq \sum_{i=1}^{5} k_{i}\right\} \text {, }
\end{aligned}
$$

and

$$
\begin{aligned}
& \left\{\left(\mathcal{I}_{+}, \mathcal{I}_{+}, \quad \sum_{i=1}^{5} k_{i}-2 \mathcal{I}_{+}\right) \mid \mathcal{I}_{+} \text {is integer and } k_{5} \leq 2 \mathcal{I}_{+} \leq \sum_{i=1}^{5} k_{i}\right\} \\
= & \left\{\mathbb{I} n\left(\left[\begin{array}{ccccc}
\mathcal{Z}_{11} & 0 & 0 & 0 & 0 \\
0 & \mathcal{Z}_{22} & 0 & 0 & 0 \\
0 & 0 & \mathcal{Z}_{33} & 0 & 0 \\
0 & 0 & 0 & \mathcal{Z}_{44} & I \\
0 & 0 & 0 & I & \mathcal{Z}_{55}
\end{array}\right]\right) \mid \mathcal{Z}_{i i}=-\mathcal{Z}_{i i}^{T} \in \mathbb{R}^{k_{i} \times k_{i}}, i=1, \ldots, 5\right\} \\
\subset & \left\{\mathbb{I} n(\mathcal{Z}) \mid \mathcal{Z}=-\mathcal{Z}^{T} \in \mathbb{R}^{\sum_{i=1}^{5} k_{i} \times \sum_{i=1}^{5} k_{i}}\right\}
\end{aligned}
$$

Copyright $@$ by SIAM. Unauthorized reproduction of this article is prohibited. 
$\subset\left\{\left(\mathcal{I}_{+}, \mathcal{I}_{+}, \sum_{i=1}^{5} k_{i}-2 \mathcal{I}_{+}\right) \mid \mathcal{I}_{+}\right.$is integer and $\left.k_{5} \leq 2 \mathcal{I}_{+} \leq \sum_{i=1}^{5} k_{i}\right\}$,

i.e., part (iii) holds;

- when $k_{1}, k_{2}$, and $k_{3}$ are all odd, or $k_{1}$ is even and $k_{2}$ and $k_{3}$ are odd, then $k_{2}+k_{3}$ is even,

$$
\begin{aligned}
& \left\{s \mid s \text { is even integer and } k_{5} \leq s \leq \sum_{i=1}^{5} k_{i}\right\} \\
= & \left\{\operatorname{rank}\left[\begin{array}{ccccc}
\mathcal{Z}_{11} & 0 & 0 & 0 & 0 \\
0 & \mathcal{Z}_{22} & \mathcal{Z}_{23} & 0 & 0 \\
0 & -\mathcal{Z}_{23}^{T} & \mathcal{Z}_{33} & 0 & 0 \\
0 & 0 & 0 & \mathcal{Z}_{44} & I \\
0 & 0 & 0 & I & \mathcal{Z}_{55}
\end{array}\right] \mid \mathcal{Z}_{i i}=-\mathcal{Z}_{i i}^{T} \in \mathbb{R}^{k_{i} \times k_{i}}, i=1, \ldots, 5\right\} \\
\subset & \left\{\operatorname{rank}(\mathcal{Z}) \mid \mathcal{Z}=-\mathcal{Z}^{T} \in \mathbb{R}^{\sum_{i=1}^{5} k_{i} \times \sum_{i=1}^{5} k_{i}}\right\} \\
\subset & \left\{s \mid s \text { is even integer and } k_{5} \leq s \leq \sum_{i=1}^{5} k_{i}\right\},
\end{aligned}
$$

and

$$
\begin{aligned}
& \left\{\left(\mathcal{I}_{+}, \mathcal{I}_{+}, \sum_{i=1}^{5} k_{i}-2 \mathcal{I}_{+}\right) \mid \mathcal{I}_{+} \text {is integer and } k_{5} \leq 2 \mathcal{I}_{+} \leq \sum_{i=1}^{5} k_{i}\right\} \\
= & \left\{\mathbb{I n}\left(\left[\begin{array}{ccccc}
\mathcal{Z}_{11} & 0 & 0 & 0 & 0 \\
0 & \mathcal{Z}_{22} & \mathcal{Z}_{23} & 0 & 0 \\
0 & -\mathcal{Z}_{23}^{T} & \mathcal{Z}_{33} & 0 & 0 \\
0 & 0 & 0 & \mathcal{Z}_{44} & I \\
0 & 0 & 0 & I & \mathcal{Z}_{55}
\end{array}\right]\right) \mid \mathcal{Z}_{i i}=-\mathcal{Z}_{i i}^{T} \in \mathbb{R}^{k_{i} \times k_{i}}, i=1, \ldots, 5\right\} \\
\subset & \left\{\mathbb{I} n(\mathcal{Z}) \mid \mathcal{Z}=-\mathcal{Z}^{T} \in \mathbb{R}^{\sum_{i=1}^{5} k_{i} \times \sum_{i=1}^{5} k_{i}}\right\} \\
\subset & \left\{\left(\mathcal{I}_{+}, \mathcal{I}_{+}, \sum_{i=1}^{5} k_{i}-2 \mathcal{I}_{+}\right) \mid \mathcal{I}_{+} \text {is integer and } k_{5} \leq 2 \mathcal{I}_{+} \leq \sum_{i=1}^{5} k_{i}\right\},
\end{aligned}
$$

i.e., part (iii) holds;

- when $k_{3}$ is even and $k_{1}$ and $k_{2}$ are odd, then $k_{1}+k_{2}$ is even,

$$
\begin{aligned}
& \left\{s \mid s \text { is even integer and } k_{5} \leq s \leq \sum_{i=1}^{5} k_{i}\right\} \\
= & \left\{\operatorname{rank}\left[\begin{array}{ccccc}
\mathcal{Z}_{11} & \mathcal{Z}_{12} & 0 & 0 & 0 \\
-\mathcal{Z}_{12}^{T} & \mathcal{Z}_{22} & 0 & 0 & 0 \\
0 & 0 & \mathcal{Z}_{33} & 0 & 0 \\
0 & 0 & 0 & \mathcal{Z}_{44} & I \\
0 & 0 & 0 & I & \mathcal{Z}_{55}
\end{array}\right] \mid \mathcal{Z}_{i i}=-\mathcal{Z}_{i i}^{T} \in \mathbb{R}^{k_{i} \times k_{i}}, i=1, \ldots, 5\right\} \\
\subset & \left\{\operatorname{rank}(\mathcal{Z}) \mid \mathcal{Z}=-\mathcal{Z}^{T} \in \mathbb{R}^{\sum_{i=1}^{5}} k_{i} \times \sum_{i=1}^{5} k_{i}\right\} \\
\subset & \left\{s \mid s \text { is even integer and } k_{5} \leq s \leq \sum_{i=1}^{5} k_{i}\right\}
\end{aligned}
$$


and

$$
\begin{aligned}
& \left\{\left(\mathcal{I}_{+}, \mathcal{I}_{+}, \sum_{i=1}^{5} k_{i}-2 \mathcal{I}_{+}\right) \mid \mathcal{I}_{+} \text {is integer and } k_{5} \leq 2 \mathcal{I}_{+} \leq \sum_{i=1}^{5} k_{i}\right\} \\
= & \left\{\mathbb{I} n\left(\left[\begin{array}{ccccc}
\mathcal{Z}_{11} & \mathcal{Z}_{12} & 0 & 0 & 0 \\
-\mathcal{Z}_{12}^{T} & \mathcal{Z}_{22} & 0 & 0 & 0 \\
0 & 0 & \mathcal{Z}_{33} & 0 & 0 \\
0 & 0 & 0 & \mathcal{Z}_{44} & I \\
0 & 0 & 0 & I & \mathcal{Z}_{55}
\end{array}\right]\right) \mid \mathcal{Z}_{i i}=-\mathcal{Z}_{i i}^{T} \in \mathbb{R}^{k_{i} \times k_{i}}, i=1, \ldots, 5\right\} \\
\subset & \left\{\mathbb{I} n(\mathcal{Z}) \mid \mathcal{Z}=-\mathcal{Z}^{T} \in \mathbb{R}^{\sum_{i=1}^{5}} k_{i} \times \sum_{i=1}^{5} k_{i}\right. \\
\subset & \left\{\left(\mathcal{I}_{+}, \mathcal{I}_{+}, \sum_{i=1}^{5} k_{i}-2 \mathcal{I}_{+}\right) \mid \mathcal{I}_{+} \text {is integer and } k_{5} \leq 2 \mathcal{I}_{+} \leq \sum_{i=1}^{5} k_{i}\right\},
\end{aligned}
$$

i.e., part (iii) holds;

- when $k_{2}$ is even, $k_{1}$ and $k_{3}$ are odd,

- if $k_{2}>0$, then

$$
\begin{aligned}
& \left\{s \mid s \text { is even integer and } k_{5} \leq s \leq \sum_{i=1}^{5} k_{i}\right\} \\
& =\left\{\operatorname{rank}\left[\begin{array}{ccccccc}
\mathcal{Z}_{11} & \mathcal{Z}_{12}^{(1)} & 0 & 0 & 0 & 0 & \\
-\left(\mathcal{Z}_{12}^{(1)}\right)^{T} & 0 & 0 & 0 & 0 & 0 & 0 \\
0 & 0 & \mathcal{Z}_{22}^{(2)} & 0 & 0 & 0 & 0 \\
0 & 0 & 0 & 0 & \mathcal{Z}_{23}^{(1)} & 0 & 0 \\
0 & 0 & 0 & -\left(\mathcal{Z}_{23}^{1)}\right)^{T} & \mathcal{Z}_{33} & 0 & 0 \\
0 & 0 & 0 & 0 & 0 & \mathcal{Z}_{44} & I \\
0 & 0 & 0 & 0 & 0 & I & \mathcal{Z}_{55}
\end{array}\right]\right. \\
& \left.\mid \mathcal{Z}_{22}^{(2)}=-\left(\mathcal{Z}_{22}^{(2)}\right)^{T} \in \mathbb{R}^{\left(k_{2}-1\right) \times(k-2-1)}, \mathcal{Z}_{i i}=-\mathcal{Z}_{i i}^{T} \in \mathbb{R}^{k_{i} \times k_{i}}, i=1,3,4,5\right\} \\
& \subset\left\{\operatorname{rank}(\mathcal{Z}) \mid \mathcal{Z}=-\mathcal{Z}^{T} \in \mathbb{R}^{\sum_{i=1}^{5} k_{i} \times \sum_{i=1}^{5} k_{i}}\right\} \\
& \subset\left\{s \mid s \text { is even integer and } k_{5} \leq s \leq \sum_{i=1}^{5} k_{i}\right\} \text {, }
\end{aligned}
$$

and

$$
\left\{\left(\mathcal{I}_{+}, \mathcal{I}_{+}, \sum_{i=1}^{5} k_{i}-2 \mathcal{I}_{+}\right) \mid \mathcal{I}_{+} \text {is integer and } k_{5} \leq 2 \mathcal{I}_{+} \leq \sum_{i=1}^{5} k_{i}\right\}
$$




$$
=\left\{\operatorname{In}\left(\left[\begin{array}{ccccccc}
\mathcal{Z}_{11} & \mathcal{Z}_{12}^{(1)} & 0 & 0 & 0 & 0 & \\
-\left(\mathcal{Z}_{12}^{(1)}\right)^{T} & 0 & 0 & 0 & 0 & 0 & 0 \\
0 & 0 & \mathcal{Z}_{22}^{(2)} & 0 & 0 & 0 & 0 \\
0 & 0 & 0 & 0 & \mathcal{Z}_{23}^{(1)} & 0 & 0 \\
0 & 0 & 0 & -\left(\mathcal{Z}_{23}^{1)}\right)^{T} & \mathcal{Z}_{33} & 0 & 0 \\
0 & 0 & 0 & 0 & 0 & \mathcal{Z}_{44} & I \\
0 & 0 & 0 & 0 & 0 & I & \mathcal{Z}_{55}
\end{array}\right]\right)\right.
$$$$
\left.\mid \mathcal{Z}_{22}^{(2)}=-\left(\mathcal{Z}_{22}^{(2)}\right)^{T} \in \mathbb{R}^{\left(k_{2}-1\right) \times(k-2-1)}, \mathcal{Z}_{i i}=-\mathcal{Z}_{i i}^{T} \in \mathbb{R}^{k_{i} \times k_{i}}, i=1,3,4,5\right\}
$$

$\subset\left\{\mathbb{I} n(\mathcal{Z}) \mid \mathcal{Z}=-\mathcal{Z}^{T} \in \mathbb{R}^{\sum_{i=1}^{5} k_{i} \times \sum_{i=1}^{5} k_{i}}\right\}$

$\subset\left\{\left(\mathcal{I}_{+}, \mathcal{I}_{+}, \sum_{i=1}^{5} k_{i}-2 \mathcal{I}_{+}\right) \mid \mathcal{I}_{+}\right.$is integer and $\left.k_{5} \leq 2 \mathcal{I}_{+} \leq \sum_{i=1}^{5} k_{i}\right\}$,

i.e., part (iii) holds;

- if $k_{2}=0$

* when $k_{5}=0$, then $\mathcal{Z}$ is reduced to the following form:

$$
\mathcal{Z}=\left[\begin{array}{cc}
\mathcal{Z}_{11} & 0 \\
0 & \mathcal{Z}_{33}
\end{array}\right], \quad \mathcal{Z}_{11}=-\mathcal{Z}_{11}^{T} \in \mathbb{R}^{k_{1} \times k_{1}}, \mathcal{Z}_{33}=-\mathcal{Z}_{33}^{T} \in \mathbb{R}^{k_{3} \times k_{3}}
$$

so

$$
\begin{aligned}
& \left\{s \mid s \text { is even integer and } k_{5} \leq s \leq \sum_{i=1}^{5} k_{i}-2\right\} \\
= & \left\{s \mid s \text { is even integer and } 0 \leq s \leq k_{1}+k_{3}-2\right\} \\
= & \left\{\operatorname{rank}\left(\mathcal{Z}_{11}\right)+\operatorname{rank}\left(\mathcal{Z}_{33}\right) \mid \mathcal{Z}_{11}=-\mathcal{Z}_{11}^{T} \in \mathbb{R}^{k_{1} \times k_{1}},\right. \\
& \left.\mathcal{Z}_{33}=-\mathcal{Z}_{33}^{T} \in \mathbb{R}^{k_{3} \times k_{3}}\right\} \\
= & \left\{\operatorname{rank}(\mathcal{Z}) \mid \mathcal{Z}=-\mathcal{Z}^{T} \in \mathbb{R}^{\left(k_{1}+k_{3}\right) \times\left(k_{1}+k_{3}\right)}\right\},
\end{aligned}
$$

and

$$
\begin{aligned}
& \left\{\left(\mathcal{I}_{+}, \mathcal{I}_{+}, \sum_{i=1}^{5} k_{i}-2 \mathcal{I}_{+}\right)\right. \\
& \left.\mid \mathcal{I}_{+} \text {is integer and } k_{5} \leq 2 \mathcal{I}_{+} \leq \sum_{i=1}^{5} k_{i}-2\right\} \\
= & \left\{\left(\mathcal{I}_{+}, \mathcal{I}_{+}, k_{1}+k_{3}-2 \mathcal{I}_{+}\right)\right.
\end{aligned}
$$




$$
\begin{aligned}
\left.\mid \mathcal{I}_{+} \text {is integer and } 0 \leq 2 \mathcal{I}_{+} \leq k_{1}+k_{3}-2\right\} \\
=\left\{\mathbb{I} n(\mathcal{Z}) \mid \mathcal{Z}=-\mathcal{Z}^{T} \in \mathbb{R}^{\left(k_{1}+k_{3}\right) \times\left(k_{1}+k_{3}\right)}\right\},
\end{aligned}
$$

i.e., part (iii) holds;

* when $k_{5}>0$, then $\mathcal{Z}$ is reduced to

$$
\mathcal{Z}=\left[\begin{array}{cccc}
\mathcal{Z}_{11} & 0 & 0 & \mathcal{Z}_{15} \\
0 & \mathcal{Z}_{33} & \mathcal{Z}_{34} & 0 \\
0 & -\mathcal{Z}_{34}^{T} & \mathcal{Z}_{44} & I \\
-\mathcal{Z}_{15}^{T} & 0 & -I & \mathcal{Z}_{55}
\end{array}\right], \quad \mathcal{Z}_{i i}=-\mathcal{Z}_{i i}^{T} \in \mathbb{R}^{k_{i} \times k_{i}}, i=1,3,4,5
$$

Thus,

$$
\begin{aligned}
& \left\{s \mid s \text { is even integer and } k_{5} \leq s \leq \sum_{i=1}^{5} k_{i}\right\} \\
& =\left\{s \mid s \text { is even integer and } k_{5} \leq s \leq k_{1}+k_{3}+k_{4}+k_{5}\right\} \\
& =\left\{\operatorname{rank}\left[\begin{array}{cccccccc}
\mathcal{Z}_{11}^{(1)} & 0 & 0 & 0 & 0 & 0 & 0 & 0 \\
0 & 0 & 0 & 0 & 0 & 0 & 0 & \mathcal{Z}_{15}^{(4)} \\
0 & 0 & \mathcal{Z}_{33}^{(1)} & 0 & 0 & 0 & 0 & 0 \\
0 & 0 & 0 & 0 & 0 & \mathcal{Z}_{34}^{(4)} & 0 & 0 \\
0 & 0 & 0 & 0 & \mathcal{Z}_{44}^{(1)} & \mathcal{Z}_{44}^{(1,2)} & I & 0 \\
0 & 0 & 0 & -\mathcal{Z}_{34}^{(4)}-\left(\mathcal{Z}_{44}^{(1,2)}\right)^{T} & 0 & 0 & 1 \\
0 & 0 & 0 & 0 & -I & 0 & \mathcal{Z}_{55}^{(1)} & \mathcal{Z}_{55}^{(1,2)} \\
0 & -\mathcal{Z}_{15}^{(4)} & 0 & 0 & 0 & -1 & -\left(\mathcal{Z}_{55}^{(1,2)}\right)^{T} & 0
\end{array}\right]\right. \\
& \left.\mid \mathcal{Z}_{15}^{(4)}, \mathcal{Z}_{34}^{(4)} \in \mathbb{R}, \mathcal{Z}_{i i}^{(1)}=-\left(\mathbb{Z}_{i i}^{(1)}\right)^{T} \in \mathbb{R}^{\left(k_{i}-1\right) \times\left(k_{i}-1\right)}, i=1,3,4,5\right\} \\
& \subset\left\{\operatorname{rank}(\mathcal{Z}) \mid \mathcal{Z}=-\mathcal{Z}^{T} \in \mathbb{R}^{\left(k_{1}+k_{3}+k_{4}+k_{5}\right) \times\left(k_{1}+k_{3}+k_{4}+k_{5}\right)}\right\} \\
& \subset\left\{s \mid s \text { is even integer and } k_{5} \leq s \leq k_{1}+k_{3}+k_{4}+k_{5}\right\} \text {, }
\end{aligned}
$$

and

$$
\begin{aligned}
& \left\{\left(\mathcal{I}_{+}, \mathcal{I}_{+}, \sum_{i=1}^{5} k_{i}-2 \mathcal{I}_{+}\right) \mid k_{5} \leq 2 \mathcal{I}_{+} \leq \sum_{i=1}^{5} k_{i}\right\} \\
= & \left\{\left(\mathcal{I}_{+}, \mathcal{I}_{+}, k_{1}+k_{3}+k_{4}+k_{5}-2 \mathcal{I}_{+}\right) \mid k_{5} \leq 2 \mathcal{I}_{+} \leq k_{1}+k_{3}+k_{4}+k_{5}\right\}
\end{aligned}
$$




$$
\begin{aligned}
& =\left\{\mathbb{I n}\left(\left[\begin{array}{cccccccc}
\mathcal{Z}_{11}^{(1)} & 0 & 0 & 0 & 0 & 0 & 0 & 0 \\
0 & 0 & 0 & 0 & 0 & 0 & 0 & \mathcal{Z}_{15}^{(4)} \\
0 & 0 & \mathcal{Z}_{33}^{(1)} & 0 & 0 & 0 & 0 & 0 \\
0 & 0 & 0 & 0 & 0 & \mathcal{Z}_{34}^{(4)} & 0 & 0 \\
0 & 0 & 0 & 0 & \mathcal{Z}_{44}^{(1)} & \mathcal{Z}_{44}^{(1,2)} & I & 0 \\
0 & 0 & 0 & -\mathcal{Z}_{34}^{(4)} & -\left(\mathcal{Z}_{44}^{(1,2)}\right)^{T} & 0 & 0 & 1 \\
0 & 0 & 0 & 0 & -I & 0 & \mathcal{Z}_{55}^{(1)} & \mathcal{Z}_{55}^{(1,2)} \\
0 & -\mathcal{Z}_{15}^{(4)} & 0 & 0 & 0 & -1 & -\left(\mathcal{Z}_{55}^{(1,2)}\right)^{T} & 0
\end{array}\right]\right)\right. \\
& \left.\mid \mathcal{Z}_{15}^{(4)}, \mathcal{Z}_{34}^{(4)} \in \mathbb{R}, \mathcal{Z}_{i i}^{(1)}=-\left(\mathbb{Z}_{i i}^{(1)}\right)^{T} \in \mathbb{R}^{\left(k_{i}-1\right) \times\left(k_{i}-1\right)}, i=1,3,4,5\right\} \\
& \subset\left\{\operatorname{In}(\mathcal{Z}) \mid \mathcal{Z}=-\mathcal{Z}^{T} \in \mathbb{R}^{\left(k_{1}+k_{3}+k_{4}+k_{5}\right) \times\left(k_{1}+k_{3}+k_{4}+k_{5}\right)}\right\} \\
& \subset\left\{\left(\mathcal{I}_{+}, \mathcal{I}_{+}, \quad \sum_{i=1}^{5} k_{i}-2 \mathcal{I}_{+}\right) \mid k_{5} \leq 2 \mathcal{I}_{+} \leq \sum_{i=1}^{5} k_{i}\right\},
\end{aligned}
$$

i.e., part (iii) follows.

Hence, part (iii) is proved.

Here it should be pointed out that a simple construction method, which takes the entries of $\mathcal{Z}$ to be either 1 or 0 such that $\mathcal{Z}$ achieves any given admissible rank or inertia, is embedded in the proof of Lemma 7.

We are now ready to prove Theorem 5 .

Proof of Theorem 5. First, we can find nonsingular matrices $\mathcal{L}_{1} \in \mathbb{F}^{(n+m+p) \times(n+m+p)}$, $\mathcal{L}_{2} \in \mathbb{F}^{m \times m}$, and $\mathcal{L}_{3} \in \mathbb{F}^{p \times p}$, and two constant matrices $\mathcal{X}_{0} \in \mathbb{F}^{m \times m}$ and $\mathcal{Y}_{0} \in \mathbb{F}^{p \times p}$ by using the technique in [7] such that

$$
\begin{aligned}
& \mathcal{L}_{1}\left[\begin{array}{ccc}
\mathcal{A} & \mathcal{B} & \mathcal{C} \\
\pm \mathcal{B}^{\star} & \mathcal{X} & \mathcal{E} \\
\pm \mathcal{C}^{\star} & \pm \mathcal{E}^{\star} & \mathcal{Y}
\end{array}\right] \mathcal{L}_{1}^{\star} \\
& =\left[\begin{array}{ccccc|cccc|cccc}
\Sigma_{1} & 0 & 0 & 0 & 0 & 0 & 0 & 0 & 0 & 0 & 0 & 0 & 0 \\
0 & 0 & 0 & 0 & 0 & I_{n_{2}} & 0 & 0 & 0 & 0 & I_{n_{2}} & 0 & 0 \\
0 & 0 & 0 & 0 & 0 & 0 & I_{n_{3}} & 0 & 0 & 0 & 0 & 0 & 0 \\
0 & 0 & 0 & 0 & 0 & 0 & 0 & 0 & 0 & I_{n_{4}} & 0 & 0 & 0 \\
0 & 0 & 0 & 0 & 0 & 0 & 0 & 0 & 0 & 0 & 0 & 0 & 0 \\
\hline 0 & \pm I_{n_{2}} & 0 & 0 & 0 & \mathcal{X}_{11} & \mathcal{X}_{12} & \mathcal{X}_{13} & \mathcal{X}_{14} & 0 & 0 & 0 & 0 \\
0 & 0 & \pm I_{n_{3}} & 0 & 0 & \pm \mathcal{X}_{12}^{\star} & \mathcal{X}_{22} & \mathcal{X}_{23} & \mathcal{X}_{24} & 0 & 0 & 0 & 0 \\
0 & 0 & 0 & 0 & 0 & \pm \mathcal{X}_{13}^{\star} & \pm \mathcal{X}_{23}^{\star} & \mathcal{X}_{33} & \mathcal{X}_{34} & 0 & 0 & I_{n_{5}} & 0 \\
0 & 0 & 0 & 0 & 0 & \pm \mathcal{X}_{14}^{\star} & \pm \mathcal{X}_{24}^{\star} & \pm \mathcal{X}_{34}^{\star} & \mathcal{X}_{44} & 0 & 0 & 0 & 0 \\
\hline 0 & 0 & 0 & \pm I_{n_{4}} & 0 & 0 & 0 & 0 & 0 & \mathcal{Y}_{11} & \mathcal{Y}_{12} & \mathcal{Y}_{13} & \mathcal{Y}_{14} \\
0 & \pm I_{n_{2}} & 0 & 0 & 0 & 0 & 0 & 0 & 0 & \pm \mathcal{Y}_{12}^{\star} & \mathcal{Y}_{22} & \mathcal{Y}_{23} & \mathcal{Y}_{24} \\
0 & 0 & 0 & 0 & 0 & 0 & 0 & \pm I_{n_{5}} & 0 & \pm \mathcal{Y}_{13}^{\star} & \pm \mathcal{Y}_{23}^{\star} & \mathcal{Y}_{33} & \mathcal{Y}_{34} \\
0 & 0 & 0 & 0 & 0 & 0 & 0 & 0 & 0 & \pm \mathcal{Y}_{14}^{\star} & \pm \mathcal{Y}_{24}^{\star} & \pm \mathcal{Y}_{34}^{\star} & \mathcal{Y}_{44}
\end{array}\right],
\end{aligned}
$$


where

$$
\begin{aligned}
& \Sigma_{1} \in \mathbb{R}^{n_{1} \times n_{1}}, \quad \operatorname{rank}\left(\Sigma_{1}\right)=n_{1}, \quad \mathbb{I} n(\mathcal{A})=\mathbb{I} n\left(\Sigma_{1}\right)+\left(\begin{array}{ll}
0, & \left.0, n-n_{1}\right), \\
n_{1}=\operatorname{rank}(\mathcal{A}), & n_{1}+n_{2}+n_{3}=\operatorname{rank}\left[\begin{array}{cc}
\mathcal{A} & \mathcal{B}
\end{array}\right], \quad n_{1}+n_{2}+n_{4}=\operatorname{rank}\left[\begin{array}{ll}
\mathcal{A} & \mathcal{C}
\end{array}\right], \\
n_{1}+n_{2}+n_{3}+n_{4}=\operatorname{rank}\left[\begin{array}{lll}
\mathcal{A} & \mathcal{B} & \mathcal{C}
\end{array}\right], \quad n_{1}+2 n_{2}+n_{3}+n_{4}+n_{5}=\operatorname{rank}\left[\begin{array}{cc}
\mathcal{A} & \mathcal{C} \\
\pm \mathcal{B}^{\star} & \mathcal{E}
\end{array}\right], \\
\text { and } \\
{\left[\begin{array}{cccc}
\mathcal{X}_{11} & \mathcal{X}_{12} & \mathcal{X}_{13} & \mathcal{X}_{14} \\
\pm \mathcal{X}_{12}^{\star} & \mathcal{X}_{22} & \mathcal{X}_{23} & \mathcal{X}_{24} \\
\pm \mathcal{X}_{13}^{\star} & \pm \mathcal{X}_{23}^{\star} & \mathcal{X}_{33} & \mathcal{X}_{34} \\
\pm \mathcal{X}_{14}^{\star} & \pm \mathcal{X}_{24}^{\star} & \pm \mathcal{X}_{34}^{\star} & \mathcal{X}_{44}
\end{array}\right]=\mathcal{L}_{2}\left(\mathcal{X}+\mathcal{X}_{0}\right) \mathcal{L}_{2}^{\star},} & {\left[\begin{array}{cccc}
\mathcal{Y}_{11} & \mathcal{Y}_{12} & \mathcal{Y}_{13} & \mathcal{Y}_{14} \\
\pm \mathcal{Y}_{12}^{\star} & \mathcal{Y}_{22} & \mathcal{Y}_{23} & \mathcal{Y}_{24} \\
\pm \mathcal{Y}_{13}^{\star} & \pm \mathcal{Y}_{23}^{\star} & \mathcal{Y}_{33} & \mathcal{Y}_{34} \\
\pm \mathcal{Y}_{14}^{\star} & \pm \mathcal{Y}_{24}^{\star} & \pm \mathcal{Y}_{34}^{\star} & \mathcal{Y}_{44}
\end{array}\right]=\mathcal{L}_{3}\left(\mathcal{Y}+\mathcal{Y}_{0}\right) \mathcal{L}_{3}^{\star} .}
\end{array}\right.
\end{aligned}
$$

Clearly, it holds that

$$
\begin{aligned}
\operatorname{rank}\left[\begin{array}{ccc}
\mathcal{A} & \mathcal{B} & \mathcal{C} \\
\pm \mathcal{B}^{\star} & \mathcal{X} & \mathcal{E} \\
\pm \mathcal{C}^{\star} & \pm \mathcal{E}^{\star} & \mathcal{Y}
\end{array}\right]= & \operatorname{rank}\left[\begin{array}{ccccc}
\mathcal{X}_{33} & \mathcal{X}_{34} & \pm\left(-\mathcal{X}_{13}\right)^{\star} & I_{n_{5}} & 0 \\
\pm \mathcal{X}_{34}^{\star} & \mathcal{X}_{44} & \pm\left(-\mathcal{X}_{14}\right)^{\star} & 0 & 0 \\
-\mathcal{X}_{13} & -\mathcal{X}_{14} & \mathcal{Y}_{22}+\mathcal{X}_{11} & \mathcal{Y}_{23} & \mathcal{Y}_{24} \\
\pm I_{n_{5}} & 0 & \pm \mathcal{Y}_{23}^{\star} & \mathcal{Y}_{33} & \mathcal{Y}_{34} \\
0 & 0 & \pm \mathcal{Y}_{24}^{\star} & \pm \mathcal{Y}_{34}^{\star} & \mathcal{Y}_{44}
\end{array}\right] \\
& +n_{1}+2\left(n_{2}+n_{3}+n_{4}\right) \\
= & \\
& {\left[\begin{array}{ccccc}
\mathcal{X}_{44} & \pm\left(-\mathcal{X}_{14}\right)^{\star} & 0 & 0 & \pm \mathcal{X}_{34}^{\star} \\
-\mathcal{X}_{14} & \mathcal{Y}_{22}+\mathcal{X}_{11} & \mathcal{Y}_{24} & \mathcal{Y}_{23} & -\mathcal{X}_{13} \\
0 & \pm \mathcal{Y}_{24}^{\star} & \mathcal{Y}_{44} & \pm \mathcal{Y}_{34}^{\star} & 0 \\
0 & \pm \mathcal{Y}_{23}^{\star} & \mathcal{Y}_{34} & \mathcal{Y}_{33} & \pm I_{n_{5}} \\
\mathcal{X}_{34} & \pm\left(-\mathcal{X}_{13}\right)^{\star} & 0 & I_{n_{5}} & \mathcal{X}_{33}
\end{array}\right] } \\
& +n_{1}+2\left(n_{2}+n_{3}+n_{4}\right),
\end{aligned}
$$

and

$$
\begin{aligned}
\mathbb{I n}\left(\left[\begin{array}{ccc}
\mathcal{A} & \mathcal{B} & \mathcal{C} \\
\pm \mathcal{B}^{\star} & \mathcal{X} & \mathcal{E} \\
\pm \mathcal{C}^{\star} & \pm \mathcal{E}^{\star} & \mathcal{Y}
\end{array}\right]\right)= & \mathbb{I n}\left(\left[\begin{array}{ccccc}
\mathcal{X}_{33} & \mathcal{X}_{34} & \pm\left(-\mathcal{X}_{13}\right)^{\star} & I_{n_{5}} & 0 \\
\pm \mathcal{X}_{34}^{\star} & \mathcal{X}_{44} & \pm\left(-\mathcal{X}_{14}\right)^{\star} & 0 & 0 \\
-\mathcal{X}_{13} & -\mathcal{X}_{14} & \mathcal{Y}_{22}+\mathcal{X}_{11} & \mathcal{Y}_{23} & \mathcal{Y}_{24} \\
\pm I_{n_{5}} & 0 & \pm \mathcal{Y}_{23}^{\star} & \mathcal{Y}_{33} & \mathcal{Y}_{34} \\
0 & 0 & \pm \mathcal{Y}_{24}^{\star} & \pm \mathcal{Y}_{34}^{\star} & \mathcal{Y}_{44}
\end{array}\right]\right) \\
+ & \mathbb{I n} n\left(\Sigma_{1}\right)+\left(n_{2}+n_{3}+n_{4}, n_{2}+n_{3}+n_{4}\right. \\
& \left.n-n_{1}-n_{2}-n_{3}-n_{4}\right) \\
= & \mathbb{I n}\left(\left[\begin{array}{ccccc}
\mathcal{X}_{44} & \pm\left(-\mathcal{X}_{14}\right)^{\star} & 0 & 0 & \pm \mathcal{X}_{34}^{\star} \\
-\mathcal{X}_{14} & \mathcal{Y}_{22}+\mathcal{X}_{11} & \mathcal{Y}_{24} & \mathcal{Y}_{23} & -\mathcal{X}_{13} \\
0 & \pm \mathcal{Y}_{24}^{\star} & \mathcal{Y}_{44} & \pm \mathcal{Y}_{34}^{\star} & 0 \\
0 & \pm \mathcal{Y}_{23}^{\star} & \mathcal{Y}_{34} & \mathcal{Y}_{33} & \pm I_{n_{5}} \\
\mathcal{X}_{34} & \pm\left(-\mathcal{X}_{13}\right)^{\star} & 0 & I_{n_{5}} & \mathcal{X}_{33}
\end{array}\right]\right) \\
& +\mathbb{I n}(\mathcal{A})+\left(n_{2}+n_{3}+n_{4}, n_{2}+n_{3}+n_{4},-n_{2}-n_{3}-n_{4}\right) .
\end{aligned}
$$

Next, note that $\mathcal{Y}_{22} \in \mathbb{F}^{n_{2} \times n_{2}}, \mathcal{Y}_{44} \in \mathbb{F}^{\left(p-\left(n_{2}+n_{4}+n_{5}\right)\right) \times\left(p-\left(n_{2}+n_{4}+n_{5}\right)\right)}$, and $\mathcal{X}_{44} \in$ $\mathbb{F}^{\left(m-\left(n_{2}+n_{3}+n_{5}\right)\right) \times\left(m-\left(n_{2}+n_{3}+n_{5}\right)\right)}$; therefore, we have using Lemmas and 6 and 7 that 
- if $\mathbb{F}=\mathbb{C}$, then

$$
\left\{\operatorname{rank}\left[\begin{array}{ccc}
\mathcal{A} & \mathcal{B} & \mathcal{C} \\
\pm \mathcal{B}^{\star} & \mathcal{X} & \mathcal{E} \\
\pm \mathcal{C}^{\star} & \pm \mathcal{E}^{\star} & \mathcal{Y}
\end{array}\right] \mid \mathcal{X}= \pm \mathcal{X}^{\star} \in \mathbb{F}^{m \times m}, \mathcal{Y}= \pm \mathcal{Y}^{\star} \in \mathbb{F}^{p \times p}\right\}
$$

$=\left\{s \mid s\right.$ is integer, $\left.n_{1}+2\left(n_{2}+n_{3}+n_{4}\right)+n_{5} \leq s \leq n_{1}+n_{2}+n_{3}+n_{4}+m+p\right\}$,

and, for any given integers $\mathcal{I}_{+}$and $\mathcal{I}_{-}$, there exist matrices $\mathcal{X}= \pm \mathcal{X}^{\star} \in \mathbb{F}^{m \times m}$ and $\mathcal{Y}= \pm \mathcal{Y}^{\star} \in \mathbb{F}^{p \times p}$ such that

$$
\mathbb{I n}\left(\left[\begin{array}{ccc}
\mathcal{A} & \mathcal{B} & \mathcal{C} \\
\pm \mathcal{B}^{\star} & \mathcal{X} & \mathcal{E} \\
\pm \mathcal{C}^{\star} & \pm \mathcal{E}^{\star} & \mathcal{Y}
\end{array}\right]\right)=\left(\mathcal{I}_{+}, \mathcal{I}_{-}, n+m+p-\mathcal{I}_{+}-\mathcal{I}_{-}\right)
$$

if and only if

$$
\left\{\begin{array}{l}
\mathcal{I}_{+}(\mathcal{A})+n_{2}+n_{3}+n_{4} \leq \mathcal{I}_{+}, \\
\mathcal{I}_{-}(\mathcal{A})+n_{2}+n_{3}+n_{4} \leq \mathcal{I}_{-} \\
n_{1}+2\left(n_{2}+n_{3}+n_{4}\right)+n_{5} \leq \mathcal{I}_{+}+\mathcal{I}_{-} \leq m+p+n_{1}+n_{2}+n_{3}+n_{4}
\end{array}\right.
$$

- if $\mathbb{F}=\mathbb{R}$ and $\mathcal{A}=\mathcal{A}^{T}$, then

$$
\left\{\operatorname{rank}\left[\begin{array}{ccc}
\mathcal{A} & \mathcal{B} & \mathcal{C} \\
\mathcal{B}^{T} & \mathcal{X} & \mathcal{E} \\
\mathcal{C}^{T} & \mathcal{E}^{T} & \mathcal{Y}
\end{array}\right] \mid \mathcal{X}=\mathcal{X}^{T} \in \mathbb{R}^{m \times m}, \mathcal{Y}=\mathcal{Y}^{T} \in \mathbb{R}^{p \times p}\right\}
$$

$=\left\{s \mid s\right.$ is integer, $\left.n_{1}+2\left(n_{2}+n_{3}+n_{4}\right)+n_{5} \leq s \leq n_{1}+n_{2}+n_{3}+n_{4}+m+p\right\}$,

and, for any given integers $\mathcal{I}_{+}$and $\mathcal{I}_{-}$, there exist matrices $\mathcal{X}=\mathcal{X}^{T} \in \mathbb{R}^{m \times m}$ and $\mathcal{Y}=\mathcal{Y}^{T} \in \mathbb{R}^{p \times p}$ such that

$$
\mathbb{I n}\left(\left[\begin{array}{ccc}
\mathcal{A} & \mathcal{B} & \mathcal{C} \\
\mathcal{B}^{T} & \mathcal{X} & \mathcal{E} \\
\mathcal{C}^{T} & \mathcal{E}^{T} & \mathcal{Y}
\end{array}\right]\right)=\left(\mathcal{I}_{+}, \mathcal{I}_{-}, n+m+p-\mathcal{I}_{+}-\mathcal{I}_{-}\right)
$$

if and only if (19) holds;

- if $\mathbb{F}=\mathbb{R}$ and $\mathcal{A}=-\mathcal{A}^{T}$,

- when $n_{2}=0, n_{5}=0$, both $m-\left(n_{3}+n_{5}\right)$ and $p-\left(n_{4}+n_{5}\right)$ are odd, then

$$
\begin{aligned}
& \left\{\operatorname{rank}\left[\begin{array}{ccc}
\mathcal{A} & \mathcal{B} & \mathcal{C} \\
-\mathcal{B}^{T} & \mathcal{X} & \mathcal{E} \\
-\mathcal{C}^{T} & -\mathcal{E}^{T} & \mathcal{Y}
\end{array}\right] \mid \mathcal{X}=-\mathcal{X}^{T} \in \mathbb{R}^{m \times m}, \mathcal{Y}=-\mathcal{Y}^{T} \in \mathbb{R}^{p \times p}\right\} \\
& =\{s \mid s \text { is even integer, } \\
& \left.n_{1}+2\left(n_{2}+n_{3}+n_{4}\right)+n_{5} \leq s \leq n_{1}+n_{2}+n_{3}+n_{4}+m+p-2\right\},
\end{aligned}
$$

and, for any given integers $\mathcal{I}_{+}$and $\mathcal{I}_{-}$, there exist matrices $\mathcal{X}=-\mathcal{X}^{T} \in$ $\mathbb{R}^{m \times m}$ and $\mathcal{Y}=-\mathcal{Y}^{T} \in \mathbb{R}^{p \times p}$ such that

$$
\mathbb{I n}\left(\left[\begin{array}{ccc}
\mathcal{A} & \mathcal{B} & \mathcal{C} \\
-\mathcal{B}^{T} & \mathcal{X} & \mathcal{E} \\
-\mathcal{C}^{T} & -\mathcal{E}^{T} & \mathcal{Y}
\end{array}\right]\right)=\left(\mathcal{I}_{+}, \mathcal{I}_{-}, n+m+p-\mathcal{I}_{+}-\mathcal{I}_{-}\right)
$$

Copyright (c) by SIAM. Unauthorized reproduction of this article is prohibited. 
if and only if

$$
\begin{aligned}
& \mathcal{I}_{+}=\mathcal{I}_{-}, \\
& \quad n_{1}+2\left(n_{2}+n_{3}+n_{4}\right)+n_{5} \leq 2 \mathcal{I}_{+} \leq m+p+n_{1}+n_{2}+n_{3}+n_{4}-2 ;
\end{aligned}
$$

- otherwise,

$$
\left\{\operatorname{rank}\left[\begin{array}{ccc}
\mathcal{A} & \mathcal{B} & \mathcal{C} \\
-\mathcal{B}^{T} & \mathcal{X} & \mathcal{E} \\
-\mathcal{C}^{T} & -\mathcal{E}^{T} & \mathcal{Y}
\end{array}\right] \mid \mathcal{X}=-\mathcal{X}^{T} \in \mathbb{R}^{m \times m}, \mathcal{Y}=-\mathcal{Y}^{T} \in \mathbb{R}^{p \times p}\right\}
$$

$=\left\{s \mid s\right.$ is even integer, $\left.n_{1}+2\left(n_{2}+n_{3}+n_{4}\right)+n_{5} \leq s \leq n_{1}+n_{2}+n_{3}+n_{4}+m+p\right\}$,

and, for any given integers $\mathcal{I}_{+}$and $\mathcal{I}_{-}$, there exist matrices $\mathcal{X}=-\mathcal{X}^{T} \in$ $\mathbb{R}^{m \times m}$ and $\mathcal{Y}=-\mathcal{Y}^{T} \in \mathbb{R}^{p \times p}$ such that

$$
\mathbb{I n}\left(\left[\begin{array}{ccc}
\mathcal{A} & \mathcal{B} & \mathcal{C} \\
-\mathcal{B}^{T} & \mathcal{X} & \mathcal{E} \\
-\mathcal{C}^{T} & -\mathcal{E}^{T} & \mathcal{Y}
\end{array}\right]\right)=\left(\mathcal{I}_{+}, \mathcal{I}_{-}, n+m+p-\mathcal{I}_{+}-\mathcal{I}_{-}\right)
$$

if and only if

$$
\begin{aligned}
& \mathcal{I}_{+}=\mathcal{I}_{-}, \\
& \quad n_{1}+2\left(n_{2}+n_{3}+n_{4}\right)+n_{5} \leq 2 \mathcal{I}_{+} \leq m+p+n_{1}+n_{2}+n_{3}+n_{4} .
\end{aligned}
$$

Finally, since

$$
\begin{aligned}
& n_{1}=\operatorname{rank}(\mathcal{A}) \\
& n_{2}=\operatorname{rank}\left[\begin{array}{ll}
\mathcal{A} & \mathcal{B}
\end{array}\right]+\operatorname{rank}\left[\begin{array}{ll}
\mathcal{A} & \mathcal{C}
\end{array}\right]-\operatorname{rank}(\mathcal{A})-\operatorname{rank}\left[\begin{array}{lll}
\mathcal{A} & \mathcal{B} & \mathcal{C}
\end{array}\right] \\
& n_{3}=\operatorname{rank}\left[\begin{array}{lll}
\mathcal{A} & \mathcal{B} & \mathcal{C}
\end{array}\right]-\operatorname{rank}\left[\begin{array}{cl}
\mathcal{A} & \mathcal{C}
\end{array}\right] \\
& n_{4}=\operatorname{rank}\left[\begin{array}{lll}
\mathcal{A} & \mathcal{B} & \mathcal{C}
\end{array}\right]-\operatorname{rank}\left[\begin{array}{ll}
\mathcal{A} & \mathcal{B}
\end{array}\right] \\
& n_{5}=\operatorname{rank}(\mathcal{A})+\operatorname{rank}\left[\begin{array}{cc}
\mathcal{A} & \mathcal{C} \\
-\mathcal{B}^{T} & \mathcal{E}
\end{array}\right]-\operatorname{rank}\left[\begin{array}{ll}
\mathcal{A} & \mathcal{B}
\end{array}\right]-\operatorname{rank}\left[\begin{array}{ll}
\mathcal{A} & \mathcal{C}
\end{array}\right] \\
& n_{1}+n_{2}+n_{3}+n_{4}+m+p=\operatorname{rank}\left[\begin{array}{lll}
\mathcal{A} & \mathcal{B} & \mathcal{C}
\end{array}\right]+m+p \\
& n_{1}+2\left(n_{2}+n_{3}+n_{4}\right)+n_{5}=\mathcal{K}_{\min } \\
& \mathcal{I}_{+}(\mathcal{A})+\mathcal{I}_{-}(\mathcal{A})=\operatorname{rank}(\mathcal{A})=n_{1}
\end{aligned}
$$

Theorem 5 follows.

As an application of Theorem 5, we now prove Theorem 1.

Proof of Theorem 1. It is easy to see that for any $X= \pm X^{\star} \in \mathbb{F}^{m \times m}$ and $Y= \pm Y^{\star} \in \mathbb{F}^{p \times p}$,

$$
\operatorname{rank}\left(A-B X B^{\star}-C Y C^{\star}\right)=\operatorname{rank}\left[\begin{array}{ccccc}
A & B & C & 0 & 0 \\
\pm B^{\star} & 0 & 0 & I & 0 \\
\pm C^{\star} & 0 & 0 & 0 & I \\
0 & \pm I & 0 & -X & 0 \\
0 & 0 & \pm I & 0 & -Y
\end{array}\right]-2(m+p)
$$

Copyright $@$ by SIAM. Unauthorized reproduction of this article is prohibited. 
and

$$
\mathbb{I} n\left(A-B X B^{\star}-C Y C^{\star}\right)=\mathbb{I n}\left[\begin{array}{ccccc}
A & B & C & 0 & 0 \\
\pm B^{\star} & 0 & 0 & I & 0 \\
\pm C^{\star} & 0 & 0 & 0 & I \\
0 & \pm I & 0 & -X & 0 \\
0 & 0 & \pm I & 0 & -Y
\end{array}\right]-(m+p, m+p, 0) .
$$

Hence, Theorem 1 follows trivially from Theorem 5 with

$$
\mathcal{A}=\left[\begin{array}{ccc}
A & B & C \\
\pm B^{\star} & 0 & 0 \\
\pm C^{\star} & 0 & 0
\end{array}\right], \mathcal{B}=\left[\begin{array}{l}
0 \\
I \\
0
\end{array}\right], \mathcal{C}=\left[\begin{array}{l}
0 \\
0 \\
I
\end{array}\right], \mathcal{E}=0, X=-\mathcal{X}, Y=-\mathcal{Y}
$$

3. Alternative proof of Theorem 1. The proof of Theorem 1 given in section 2 is simple, but it is based on nonsingular transformations, not unitary/orthogonal transformations. Hence, this proof cannot be used for the purpose of numerical computing [9].

In this section, we will provide an alternative proof for Theorem 1. This alternative proof is constructive so that the method of construction in the proof can be directly translated into a procedure for computing $X$ and $Y$ that enable the ranks or inertias of the matrix expression concerned to attain any integer $s$ or triplet $\left(\mathcal{I}_{+}, \mathcal{I}_{-}, n-\mathcal{I}_{+}-\mathcal{I}_{-}\right)$within the admissible ranges. Furthermore, we have taken care to employ only unitary/orthogonal transformations in our proof. This accounts for the algebraic complexity of the alternative proof, but the corresponding procedure for computing $X$ and $Y$ is numerically reliable.

The following lemmas play an important role for the development in this section.

Lemma 8 (see [3]). Given $A_{1} \in \mathbb{F}^{\nu_{1} \times \nu_{2}}$ and $A_{2} \in \mathbb{F}^{\nu_{2} \times \nu_{2}}$ with $A_{2}$ nonsingular. Let unitary matrix $\mathcal{W} \in \mathbb{F}^{\left(\nu_{1}+\nu_{2}\right) \times\left(\nu_{1}+\nu_{2}\right)}$, with partitioning $\left.\mathcal{W}=\left[\begin{array}{cc}\nu_{1} & \nu_{2} \\ \mathcal{W}_{11} & \mathcal{W}_{12} \\ \mathcal{W}_{21} & \mathcal{W}_{22}\end{array}\right]\right\} \nu_{1}$ be such that

$$
\mathcal{W}\left[\begin{array}{c}
A_{1} \\
A_{2}
\end{array}\right]=\left[\begin{array}{c}
0 \\
\tilde{A}_{2}
\end{array}\right] \begin{gathered}
\} \nu_{1} \\
\} \nu_{2}
\end{gathered}
$$

Then both $\mathcal{W}_{11}$ and $\mathcal{W}_{22}$ are nonsingular.

Lemma 9. Let $A= \pm A^{\star} \in \mathbb{F}^{n \times n}, B \in \mathbb{F}^{n \times m}$, and $C \in \mathbb{F}^{n \times p}$. There exist unitary matrices $U \in \mathbb{F}^{n \times n}, W_{B} \in \mathbb{F}^{m \times m}$, and $W_{C} \in \mathbb{F}^{p \times p}$ such that

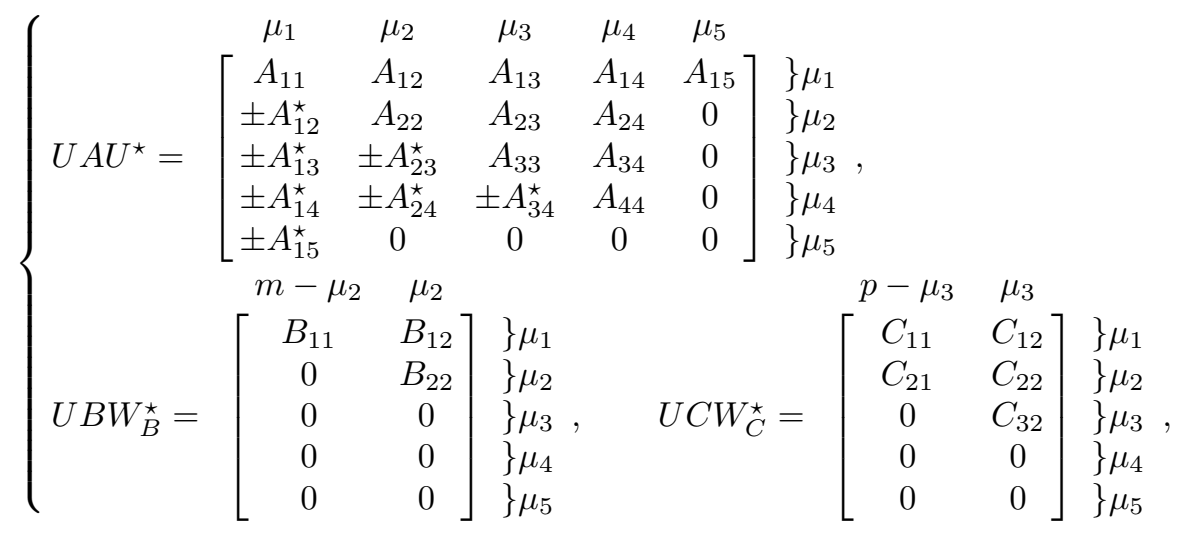


where

$$
\begin{gathered}
\operatorname{rank}\left(A_{15}\right)=\mu_{1}, \quad \operatorname{rank}\left(A_{44}\right)=\mu_{4}, \\
\operatorname{rank}\left(B_{22}\right)=\mu_{2}, \quad \operatorname{rank}\left(C_{32}\right)=\mu_{3}, \quad \operatorname{rank}\left[\begin{array}{cccc}
B_{11} & B_{12} & C_{11} & C_{12} \\
0 & B_{22} & C_{21} & C_{22} \\
0 & 0 & 0 & C_{32}
\end{array}\right]=\mu_{1}+\mu_{2}+\mu_{3}, \\
A_{11}= \pm A_{11}^{\star}, \quad A_{22}= \pm A_{22}^{\star}, \quad A_{33}= \pm A_{33}^{\star}, \quad A_{44}= \pm A_{44}^{\star},
\end{gathered}
$$

and moreover,

$$
\left\{\begin{aligned}
\mu_{1} & =\operatorname{rank}\left[\begin{array}{cc}
B & C
\end{array}\right]+\operatorname{rank}\left[\begin{array}{ccc}
A & B & C
\end{array}\right]-\operatorname{rank}\left[\begin{array}{ccc}
A & B & C \\
B^{\star} & 0 & 0 \\
C^{\star} & 0 & 0
\end{array}\right], \\
\mu_{2} & =\operatorname{rank}\left[\begin{array}{ccc}
A & B & C \\
B^{\star} & 0 & 0
\end{array}\right]-\operatorname{rank}\left[\begin{array}{ccc}
A & B & C
\end{array}\right] \\
\mu_{3} & =\operatorname{rank}\left[\begin{array}{ccc}
A & B & C \\
B^{\star} & 0 & 0 \\
C^{\star} & 0 & 0
\end{array}\right]-\operatorname{rank}\left[\begin{array}{ccc}
A & B & C \\
B^{\star} & 0 & 0
\end{array}\right], \\
\mu_{4} & =\operatorname{rank}\left[\begin{array}{ccc}
A & B & C \\
B^{\star} & 0 & 0 \\
C^{\star} & 0 & 0
\end{array}\right]-2 \operatorname{rank}\left[\begin{array}{cc}
B & C
\end{array}\right],
\end{aligned}\right.
$$

and

$$
\left\{\begin{array}{l}
\mathcal{I}_{+}\left(A_{44}\right)=\mathcal{I}_{+}\left(\left[\begin{array}{ccc}
A & B & C \\
B^{\star} & 0 & 0 \\
C^{\star} & 0 & 0
\end{array}\right]\right)-\left(\mu_{1}+\mu_{2}+\mu_{3}\right), \\
\mathcal{I}_{-}\left(A_{44}\right)=\mathcal{I}_{-}\left(\left[\begin{array}{ccc}
A & B & C \\
B^{\star} & 0 & 0 \\
C^{\star} & 0 & 0
\end{array}\right]\right)-\left(\mu_{1}+\mu_{2}+\mu_{3}\right), \\
\mathcal{I}_{+}\left(A_{44}\right)+\mathcal{I}_{-}\left(A_{44}\right)=\mu_{4} .
\end{array}\right.
$$

Proof. We construct the form (23) by the following steps:

Step 1: Compute unitary matrix $U \in \mathbb{F}^{n \times n}$ such that

$$
\left.U\left[\begin{array}{cc}
B & C
\end{array}\right]=:\left[\begin{array}{cc}
m & p \\
B_{1}^{(1)} & C_{1}^{(1)} \\
0 & 0
\end{array}\right]\right\} r \quad r, \quad \operatorname{rank}\left[\begin{array}{ll}
B_{1}^{(1)} & C_{1}^{(1)}
\end{array}\right]=r,
$$

where

$$
r=\operatorname{rank}\left[\begin{array}{cc}
B & C
\end{array}\right] .
$$

Denote

$$
U A U^{\star}=:\left[\begin{array}{cc}
r & n-r \\
A_{11}^{(1)} & A_{12}^{(1)} \\
\pm\left(A_{12}^{(1)}\right)^{\star} & A_{22}^{(1)}
\end{array}\right] \begin{aligned}
& \} r \\
& \} n-r
\end{aligned}
$$

Copyright $@$ by SIAM. Unauthorized reproduction of this article is prohibited. 
Step 2: Compute unitary matrix $U_{1} \in \mathbb{F}^{(n-r) \times(n-r)}$ such that

$$
\begin{gathered}
\mu_{4} \\
\mu_{5} \\
\left.U_{1} A_{22}^{(1)}=\left[\begin{array}{cc}
A_{44}^{(2)} & A_{45}^{(2)} \\
0 & 0
\end{array}\right]\right\} \mu_{4}, \quad \operatorname{rank}\left[\begin{array}{cc}
A_{44}^{(2)} & A_{45}^{(2)}
\end{array}\right]=\mu_{4} .
\end{gathered}
$$

Note that $A= \pm A^{\star}$, so we have $A_{22}^{(1)}= \pm\left(A_{22}^{(1)}\right)^{\star}$ as a result, we obtain

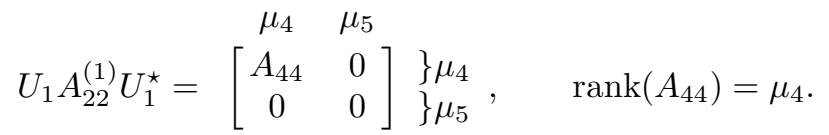

Let

$$
U:=\left[\begin{array}{ll}
I & \\
& U_{1}
\end{array}\right] U
$$

By taking the fact $A= \pm A^{\star}$ into account, we have

$$
\begin{aligned}
U A U^{\star} *= & {\left[\begin{array}{ll}
I & \\
& U_{1}
\end{array}\right]\left[\begin{array}{ccc}
A_{11}^{(1)} & A_{12}^{(1)} \\
\pm\left(A_{12}^{(1)}\right)^{\star} & A_{22}^{(1)}
\end{array}\right]\left[\begin{array}{ll}
I & \\
& U_{1}
\end{array}\right]^{\star} } \\
= & \left.:\left[\begin{array}{ccc}
r & \mu_{4} & \mu_{5} \\
A_{11}^{(2)} & A_{14}^{(2)} & A_{15}^{(2)} \\
\pm\left(A_{14}^{(2)}\right)^{\star} & A_{44} & 0 \\
\pm\left(A_{15}^{(2)}\right)^{\star} & 0 & 0
\end{array}\right]\right\} \mu_{4} \\
&
\end{aligned}
$$

and

$$
\begin{aligned}
U\left[\begin{array}{ll}
B & C
\end{array}\right] & =\left[\begin{array}{ll}
I & \\
& U_{1}
\end{array}\right]\left[\begin{array}{cc}
B_{1}^{(1)} & C_{1}^{(1)} \\
0 & 0
\end{array}\right] \\
& =:\left[\begin{array}{cc}
B_{1}^{(2)} & C_{1}^{(2)} \\
0 & 0 \\
0 & 0
\end{array}\right], \quad B_{1}^{(2)}=B_{1}^{(1)}, \quad C_{1}^{(2)}=C_{1}^{(1)} .
\end{aligned}
$$

Step 3: Compute unitary matrix $U_{2} \in \mathbb{F}^{r \times r}$ such that

$$
U_{2} A_{15}^{(2)}=\left[\begin{array}{c}
A_{15} \\
0
\end{array}\right] \begin{aligned}
& \} \mu_{1} \\
& \} r-\mu_{1}
\end{aligned}, \quad \operatorname{rank}\left(A_{15}\right)=\mu_{1} .
$$

Define

$$
U:=\left[\begin{array}{ll}
U_{2} & \\
& I
\end{array}\right] U
$$

Again, since $A= \pm A^{\star}$ and $\operatorname{rank}\left[\begin{array}{ll}B_{1}^{(2)} & C_{1}^{(2)}\end{array}\right]=\operatorname{rank}\left[\begin{array}{ll}B_{1}^{(1)} & C_{1}^{(1)}\end{array}\right]=r$ we have

$$
U A U^{\star}=\left[\begin{array}{ll}
U_{2} & \\
& I
\end{array}\right]\left[\begin{array}{ccc}
A_{11}^{(2)} & A_{14}^{(2)} & A_{15}^{(2)} \\
\pm\left(A_{14}^{(2)}\right)^{\star} & A_{44} & 0 \\
\pm\left(A_{15}^{(2)}\right)^{\star} & 0 & 0
\end{array}\right]\left[\begin{array}{ll}
U_{2} & \\
& I
\end{array}\right]^{\star}
$$

Copyright (c) by SIAM. Unauthorized reproduction of this article is prohibited. 


$$
\begin{gathered}
\left.=\left[\begin{array}{cccc}
\mu_{1} & r-\mu_{1} & \mu_{4} & \mu_{5} \\
A_{11} & A_{12}^{(3)} & A_{14} & A_{15} \\
\pm\left(A_{12}^{(3)}\right)^{\star} & A_{22}^{(3)} & A_{24}^{(3)} & 0 \\
\pm A_{14}^{\star} & \pm\left(A_{24}^{(3)}\right)^{\star} & A_{44} & 0 \\
\pm A_{15}^{\star} & 0 & 0 & 0
\end{array}\right]\right\} \mu_{1} \\
\} r-\mu_{1} \\
\} \mu_{4} \\
\mu_{5}
\end{gathered}
$$

and

$\operatorname{rank}\left[\begin{array}{cc}B_{1}^{(3)} & C_{1}^{(3)} \\ B_{2}^{(3)} & C_{2}^{(3)}\end{array}\right]=\operatorname{rank}\left(U_{2}\left[\begin{array}{ll}B_{1}^{(2)} & C_{1}^{(2)}\end{array}\right]\right)=\operatorname{rank}\left[\begin{array}{ll}B_{1}^{(1)} & C_{1}^{(1)}\end{array}\right]=r$.

Step 4: Since rank $\left[\begin{array}{cc}B_{2}^{(3)} & C_{2}^{(3)}\end{array}\right]=r-\mu_{1}$, we can compute unitary matrices $U_{4} \in \mathbb{F}^{\left(r-\mu_{1}\right) \times\left(r-\mu_{1}\right)}, W_{B} \in \mathbb{F}^{m \times m}$, and $W_{C} \in \mathbb{F}^{p \times p}$ such that $\left.U_{4}\left[\begin{array}{ll}B_{2}^{(3)} & C_{2}^{(3)}\end{array}\right]\left[\begin{array}{ll}W_{B} & \\ & W_{C}\end{array}\right]^{\star}=:\left[\begin{array}{cccc}m-\mu_{2} & \mu_{2} & p-\mu_{3} & \mu_{3} \\ 0 & B_{22} & C_{21} & C_{22} \\ 0 & 0 & 0 & C_{32}\end{array}\right]\right\} \mu_{2}$

and

$$
\operatorname{rank}\left(B_{22}\right)=\mu_{2}, \quad \operatorname{rank}\left(C_{32}\right)=\mu_{3}, \quad \mu_{2}+\mu_{3}=r-\mu_{1} .
$$

Denote

$$
U:=\left[\begin{array}{ccc}
I_{\mu_{1}} & & \\
& U_{4} & \\
& & I
\end{array}\right] U .
$$

We have by using the property $A= \pm A^{T}$ that

$$
\begin{aligned}
& U A U^{\star}=\left[\begin{array}{lll}
I_{\mu_{1}} & & \\
& U_{4} & \\
& & I
\end{array}\right]\left[\begin{array}{cccc}
A_{11} & A_{12}^{(3)} & A_{14} & A_{15} \\
\pm\left(A_{12}^{(3)}\right)^{\star} & A_{22}^{(3)} & A_{24}^{(3)} & 0 \\
\pm A_{14}^{\star} & \pm\left(A_{24}^{(3)}\right)^{\star} & A_{44} & 0 \\
\pm A_{15}^{\star} & 0 & 0 & 0
\end{array}\right]\left[\begin{array}{lll}
I_{\mu_{1}} & & \\
& U_{4} & \\
& & I
\end{array}\right]^{\star}
\end{aligned}
$$

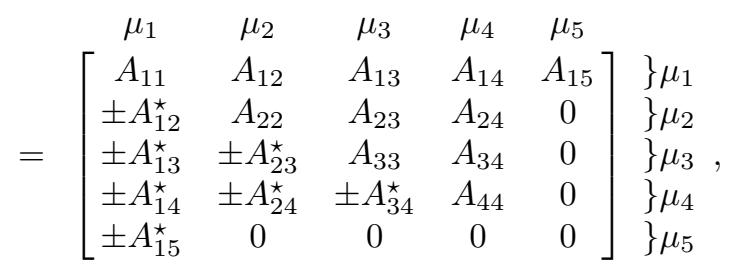

$$
\begin{aligned}
& m-\mu_{2} \quad \mu_{2} \\
& U B W_{B}^{\star}=\left[\begin{array}{lll}
I_{\mu_{1}} & & \\
& U_{4} & \\
& & I
\end{array}\right]\left[\begin{array}{c}
B_{1}^{(3)} \\
B_{2}^{(3)} \\
0 \\
0
\end{array}\right] W_{B}^{\star}=\left[\begin{array}{cc}
B_{11} & B_{12} \\
0 & B_{22} \\
0 & 0 \\
0 & 0 \\
0 & 0
\end{array}\right] \begin{array}{l}
\} \mu_{1} \\
\} \mu_{3} \\
\} \mu_{4} \\
\} \mu_{5}
\end{array}
\end{aligned}
$$


$\left.U C W_{C}^{\star}=\left[\begin{array}{llll}I_{\mu_{1}} & & \\ & U_{4} & \\ & & I\end{array}\right]\left[\begin{array}{c}C_{1}^{(3)} \\ C_{2}^{(3)} \\ 0 \\ 0\end{array}\right] W_{C}^{\star}=\left[\begin{array}{ccc}C_{11} & C_{12} \\ C_{21} & C_{22} \\ 0 & C_{32} \\ 0 & 0 \\ 0 & 0\end{array}\right]\right\} \mu_{1}$

A simple calculation yields that $\operatorname{rank}\left[\begin{array}{cccc}B_{11} & B_{12} & C_{11} & C_{12} \\ 0 & B_{22} & C_{21} & C_{22} \\ 0 & 0 & 0 & C_{32}\end{array}\right]=\operatorname{rank}\left[\begin{array}{cc}B_{1}^{(3)} & C_{1}^{(3)} \\ B_{2}^{(3)} & C_{2}^{(3)}\end{array}\right]=r=\mu_{1}+\mu_{2}+\mu_{2}$.

Furthermore, the form (23) also implies that

$$
\left\{\begin{array}{l}
\operatorname{rank}\left[\begin{array}{cc}
B & C
\end{array}\right]=r=\mu_{1}+\mu_{2}+\mu_{3}, \\
\operatorname{rank}\left[\begin{array}{ccc}
A & B & C
\end{array}\right]=2 \mu_{1}+\mu_{2}+\mu_{3}+\mu_{4}, \\
\operatorname{rank}\left[\begin{array}{ccc}
A & B & C \\
B^{\star} & 0 & 0
\end{array}\right]=2 \mu_{1}+2 \mu_{2}+\mu_{3}+\mu_{4}, \\
\operatorname{rank}\left[\begin{array}{ccc}
A & B & C \\
B^{\star} & 0 & 0 \\
C^{\star} & 0 & 0
\end{array}\right]=2 \mu_{1}+2 \mu_{2}+2 \mu_{3}+\mu_{4},
\end{array}\right.
$$

i.e., (24) holds. In addition, we also have (25).

Lemma 10. Let $A= \pm A^{\star} \in \mathbb{F}^{n \times n}, B \in \mathbb{F}^{n \times m}$, and $C \in \mathbb{F}^{n \times p}$, and let the form (23) have been determined. Let unitary matrices $V, \mathcal{V} \in \mathbb{F}^{\left(\mu_{2}+\mu_{3}+\mu_{4}\right) \times\left(\mu_{2}+\mu_{3}+\mu_{4}\right)}$ with partitioning

$$
\left.\left.V=\left[\begin{array}{ccc}
\mu_{2} & \mu_{3} & \mu_{4} \\
V_{22} & 0 & V_{24} \\
0 & I & 0 \\
V_{42} & 0 & V_{44}
\end{array}\right]\right\} \mu_{2} \quad \begin{array}{c}
\mu_{2} \\
\} \mu_{3} \\
\} \mu_{4}
\end{array}, \quad \mathcal{V}=\left[\begin{array}{ccc}
I & 0 & 0 \\
0 & \mathcal{V}_{33} & \mathcal{V}_{34} \\
0 & \mathcal{V}_{43} & \mathcal{V}_{44}
\end{array}\right]\right\} \mu_{2}
$$

be such that

$$
\left.\left.\left[\begin{array}{ll}
\mathcal{V}_{33} & \mathcal{V}_{34} \\
\mathcal{V}_{43} & \mathcal{V}_{44}
\end{array}\right]\left[\begin{array}{l}
A_{34} \\
A_{44}
\end{array}\right]=\left[\begin{array}{l}
0 \\
\Xi
\end{array}\right]\right\} \mu_{3}, \quad\left[\begin{array}{ll}
V_{22} & V_{24} \\
V_{42} & V_{44}
\end{array}\right]\left[\begin{array}{c}
A_{24} \\
\Xi
\end{array}\right]=\left[\begin{array}{c}
0 \\
\tilde{\Xi}
\end{array}\right]\right\} \mu_{2}
$$

Denote

$$
\left\{\begin{array}{c}
\left.(V \mathcal{V})\left[\begin{array}{ccc}
A_{22} & A_{23} & A_{24} \\
\pm A_{23}^{\star} & A_{33} & A_{34} \\
\pm A_{24}^{\star} & \pm A_{34}^{\star} & A_{44}
\end{array}\right](V \mathcal{V})^{\star}=\left[\begin{array}{ccc}
\mu_{2} & \mu_{3} & \mu_{4} \\
\tilde{A}_{22} & \tilde{A}_{23} & \tilde{A}_{24} \\
\pm \tilde{A}_{23}^{\star} & \tilde{A}_{33} & \tilde{A}_{34} \\
\pm \tilde{A}_{24}^{\star} & \pm \tilde{A}_{34}^{\star} & \tilde{A}_{44}
\end{array}\right]\right\} \mu_{2} \\
\left.(V \mathcal{V})\left[\begin{array}{c}
B_{22} \\
0 \\
0
\end{array}\right]=\left[\begin{array}{c}
\tilde{B}_{22} \\
0 \\
\tilde{B}_{42}
\end{array}\right]\right\} \mu_{4} \\
\} \mu_{3} \\
\} \mu_{4} \\
p-\mu_{3}
\end{array}\right.
$$

Copyright (c) by SIAM. Unauthorized reproduction of this article is prohibited. 
Furthermore, let unitary matrix $\mathcal{U} \in \mathbb{F}^{\left(\mu_{2}+\mu_{3}\right) \times\left(\mu_{2}+\mu_{3}\right)}$ be such that

$$
\mathcal{U}\left[\begin{array}{l}
\tilde{C}_{22} \\
\tilde{C}_{32}
\end{array}\right]=\left[\begin{array}{c}
0 \\
\tilde{\mathcal{C}}_{32}
\end{array}\right] \begin{gathered}
\} \mu_{2} \\
\} \mu_{3}
\end{gathered}
$$

Set

$$
\begin{gathered}
\left.\mathcal{U}\left[\begin{array}{c}
\tilde{A}_{23} \\
\tilde{A}_{33}
\end{array}\right]=\left[\begin{array}{c}
\mathcal{A}_{23} \\
\hat{\mathcal{A}}_{33}
\end{array}\right] \begin{array}{l}
\} \mu_{2} \\
\} \mu_{3}
\end{array}, \quad \mathcal{U}\left[\begin{array}{cc}
\tilde{A}_{22} & \tilde{A}_{23} \\
\pm \tilde{A}_{23}^{\star} & \tilde{A}_{33}
\end{array}\right] \mathcal{U}^{\star}=\left[\begin{array}{cc}
\mu_{2} & \mu_{3} \\
\pm \tilde{\mathcal{A}}_{23}^{\star} & \tilde{\mathcal{A}}_{23} \\
\tilde{\mathcal{A}}_{33}
\end{array}\right]\right\} \mu_{2} \\
\left.\mathcal{U}\left[\begin{array}{c}
\tilde{B}_{22} \\
0
\end{array}\right]=\left[\begin{array}{c}
\mathcal{B}_{22} \\
\tilde{\mathcal{B}}_{32}
\end{array}\right] \begin{array}{l}
\} \mu_{2} \\
\} \mu_{3}
\end{array}, \quad \mathcal{U}\left[\begin{array}{c}
\tilde{C}_{21} \\
0
\end{array}\right]=\left[\begin{array}{c}
\mathcal{C}_{21} \\
\tilde{\mathcal{C}}_{31}
\end{array}\right]\right\} \mu_{2} \\
\} \mu_{3}
\end{gathered}
$$

and define

$$
\Theta:=\left[\begin{array}{cc}
\mathcal{A}_{22} & \mathcal{A}_{23} \\
\pm \mathcal{A}_{23}^{\star} & \tilde{A}_{33}
\end{array}\right], \quad \Phi:=\left[\begin{array}{cc}
0 & \mathcal{B}_{22} \\
0 & 0
\end{array}\right], \quad \Psi=\left[\begin{array}{cc}
\mathcal{C}_{21} & 0 \\
0 & \tilde{C}_{32}
\end{array}\right] .
$$

Then $\mathcal{B}_{22}$ is nonsingular, $\Theta= \pm \Theta^{\star}$,

$$
\left\{\begin{array}{l}
\operatorname{rank}(\Phi)=\operatorname{rank}\left[\begin{array}{ccc}
A & B & C \\
B^{\star} & 0 & 0
\end{array}\right]-\operatorname{rank}\left[\begin{array}{ccc}
A & B & C
\end{array}\right], \\
\operatorname{rank}(\Psi)=\operatorname{rank}\left[\begin{array}{ccc}
A & B & C \\
C^{\star} & 0 & 0
\end{array}\right]-\operatorname{rank}\left[\begin{array}{ccc}
A & B & C
\end{array}\right], \\
\operatorname{rank}\left[\begin{array}{cc}
\Theta & \Phi \\
\Psi^{\star} & 0
\end{array}\right]=\operatorname{rank}\left[\begin{array}{cc}
A & C \\
B^{\star} & 0
\end{array}\right]+\operatorname{rank}\left[\begin{array}{ccc}
A & B & C \\
B^{\star} & 0 & 0 \\
C^{\star} & 0 & 0
\end{array}\right]-2 \operatorname{rank}\left[\begin{array}{lll}
A & B & C
\end{array}\right],
\end{array}\right.
$$

and for any $X= \pm X^{\star} \in \mathbb{F}^{m \times m}$ and $Y= \pm Y^{\star} \in \mathbb{F}^{p \times p}$,

$\operatorname{rank}\left(A-B X B^{\star}-C Y C^{\star}\right)=2 \mu_{1}+\mu_{4}+\operatorname{rank}\left(\Theta-\Phi\left(W_{B} X W_{B}^{\star}\right) \Phi^{\star}-\Psi\left(W_{C} Y W_{C}^{\star}\right) \Psi^{\star}\right)$

$(27)$

and

$$
\left\{\begin{array}{c}
\mathcal{I}_{+}\left(A-B X B^{\star}-C Y C^{\star}\right) \\
=\mu_{1}+\mathcal{I}_{+}\left(A_{44}\right)+\mathcal{I}_{+}\left(\Theta-\Phi\left(W_{B} X W_{B}^{\star}\right) \Phi^{\star}-\Psi\left(W_{C} Y W_{C}^{\star}\right) \Psi^{\star}\right), \\
\mathcal{I}_{-}\left(A-B X B^{\star}-C Y C^{\star}\right) \\
\quad=\mu_{1}+\mathcal{I}_{-}\left(A_{44}\right)+\mathcal{I}_{-}\left(\Theta-\Phi\left(W_{B} X W_{B}^{\star}\right) \Phi^{\star}-\Psi\left(W_{C} Y W_{C}^{\star}\right) \Psi^{\star}\right) .
\end{array}\right.
$$

Proof. First, since $A_{44}$ is nonsingular, so it follows directly from Lemma 8 that $V_{22}$ and $\mathcal{V}_{33}$ are nonsingular. Note that $\tilde{B}_{22}=V_{22} B_{22}, \tilde{C}_{32}=\mathcal{V}_{33} C_{32}$, and $B_{22}$ and $C_{32}$ are nonsingular; thus, $\tilde{B}_{22}$ and $\tilde{C}_{32}$ are also nonsingular.

Next, partition $\mathcal{U}$ into

$$
\begin{gathered}
\mu_{2} \\
\mu_{3} \\
\left.\mathcal{U}=\left[\begin{array}{cc}
\mathcal{U}_{22} & \mathcal{U}_{23} \\
\mathcal{U}_{32} & \mathcal{U}_{33}
\end{array}\right]\right\} \mu_{2} \\
\} \mu_{3}
\end{gathered}
$$

We get by using Lemma 8 that $\mathcal{U}_{22}$ and $\mathcal{U}_{33}$ are nonsingular because $\tilde{C}_{32}$ is nonsingular. Moreover, we also have

$$
\left\{\begin{array}{l}
\mathcal{B}_{22}=\mathcal{U}_{22} \tilde{B}_{22} \\
\Theta=\left[\begin{array}{cc}
\mathcal{U}_{22} & \mathcal{U}_{23} \\
0 & I
\end{array}\right]\left[\begin{array}{cc}
\tilde{A}_{22} & \tilde{A}_{23} \\
\pm \tilde{A}_{23}^{\star} & \tilde{A}_{33}
\end{array}\right]\left[\begin{array}{cc}
\mathcal{U}_{22} & \mathcal{U}_{23} \\
0 & I
\end{array}\right]^{\star}, \\
\Phi=\left[\begin{array}{cc}
\mathcal{U}_{22} & \mathcal{U}_{23} \\
0 & I
\end{array}\right]\left[\begin{array}{cc}
0 & \tilde{B}_{22} \\
0 & 0
\end{array}\right], \quad \Psi=\left[\begin{array}{cc}
\mathcal{U}_{22} & \mathcal{U}_{23} \\
0 & I
\end{array}\right]\left[\begin{array}{cc}
\tilde{C}_{21} & \tilde{C}_{22} \\
0 & \tilde{C}_{32}
\end{array}\right] .
\end{array}\right.
$$

Copyright (c) by SIAM. Unauthorized reproduction of this article is prohibited. 
Hence, $\mathcal{B}_{22}$ is nonsingular since $\mathcal{U}_{22}$ and $\tilde{B}_{22}$ are nonsingular. Moreover, let's rename

$$
U:=\left[\begin{array}{ccccc}
I & & & & \\
& \mathcal{U}_{22} & \mathcal{U}_{23} & & \\
& & I & & \\
& & & I_{\mu_{4}} & \\
& & & & I_{\mu_{5}}
\end{array}\right]\left[\begin{array}{ccccc}
I_{\mu_{1}} & & & & \\
& V_{22} & V_{24} \mathcal{V}_{43} & V_{24} \mathcal{V}_{44} & \\
& & \mathcal{V}_{33} & \mathcal{V}_{34} & \\
& & & I & \\
& & & & I
\end{array}\right] U .
$$

Then $U$ is nonsingular, and a simple calculation using (23) gives that $\left(U A U^{\star}, U B W_{B}^{\star}\right.$, $\left.U C W_{C}^{\star}\right)$ is of the following form:

$$
\left\{\begin{array}{c}
U A U^{\star}=\left[\begin{array}{cccc}
A_{11} & \star & A_{14} & A_{15} \\
\star & \Theta & 0 & 0 \\
\pm A_{14}^{\star} & 0 & A_{44} & 0 \\
\pm A_{15}^{\star} & 0 & 0 & 0
\end{array}\right] \\
U B W_{B}^{\star}=\left[\begin{array}{c}
\star \\
\Phi \\
0 \\
0
\end{array}\right],
\end{array}\right.
$$

which together with properties that $U$ and $A_{44}$ are nonsingular and $A_{15}$ is of full row rank leads to (27) and (28) directly. In addition, $A= \pm A^{\star}$ gives $\Theta= \pm \Theta^{\star}$, and (26) follows from (24) and the following equalities

$$
\left\{\begin{array}{l}
\operatorname{rank}(\Phi)=\operatorname{rank}\left(\mathcal{B}_{22}\right)=\mu_{2}, \\
\operatorname{rank}(\Psi)=\operatorname{rank}\left(\Psi^{\star}\right)=\operatorname{rank}\left[\begin{array}{ccc}
A & B & C \\
C^{\star} & 0 & 0
\end{array}\right]-2 \mu_{1}-\mu_{2}-\mu_{3}-\mu_{4}, \\
\operatorname{rank}\left[\begin{array}{cc}
\Theta & \Phi \\
\Psi^{\star} & 0
\end{array}\right]=\operatorname{rank}\left[\begin{array}{cc}
A & C \\
B^{\star} & 0
\end{array}\right]-2 \mu_{1}-\mu_{4} .
\end{array}\right.
$$

Lemma 11. Let $A= \pm A^{\star} \in \mathbb{F}^{n \times n}, B \in \mathbb{F}^{n \times m}$, and $C \in \mathbb{F}^{n \times p}$, and let $\Theta, \Phi$, and $\Psi$ in Lemma 10 have been determined. There exist unitary matrices $P \in$ $\mathbb{F}^{\left(\mu_{2}+\mu_{3}\right) \times\left(\mu_{2}+\mu_{3}\right)}$ and $Q \in \mathbb{F}^{p \times p}$ such that

$$
\left\{\begin{array}{c}
\left.P \Theta P^{\star}=\left[\begin{array}{ccccc}
\tau_{1} & \tau_{2} & \tau_{3} & \tau_{4} & \tau_{1} \\
\Theta_{11} & \Theta_{12} & \Theta_{13} & 0 & \Sigma \\
\pm \Theta_{12}^{\star} & \Theta_{22} & \Theta_{23} & 0 & 0 \\
\pm \Theta_{13}^{\star} & \pm \Theta_{23}^{\star} & \Theta_{33} & \Theta_{34} & \Theta_{35} \\
0 & 0 & \pm \Theta_{34}^{\star} & \Theta_{44} & \Theta_{45} \\
\pm \Sigma^{\star} & 0 & \pm \Theta_{35}^{\star} & \pm \Theta_{45}^{\star} & \Theta_{55}
\end{array}\right]\right\} \tau_{1} \\
\} \tau_{2} \\
\} \tau_{3},
\end{array}\right.
$$

where $\Theta_{i, i}= \pm \Theta_{i, i}^{\star}(i=1, \ldots, 5),\left[\begin{array}{c}\Phi_{12} \\ \Phi_{22} \\ \Phi_{32}\end{array}\right], \Psi_{32},\left[\begin{array}{l}\Psi_{43} \\ \Psi_{53}\end{array}\right]$, and $\Sigma=\left[\begin{array}{ccc}\sigma_{1} & & \\ & \ddots & \\ & & \sigma_{\tau_{1}}\end{array}\right]$ 
are nonsingular, and

$$
\begin{aligned}
\tau_{1}= & \operatorname{rank}\left[\begin{array}{cc}
A & C \\
B^{\star} & 0
\end{array}\right]+\operatorname{rank}\left[\begin{array}{ccc}
A & B & C \\
B^{\star} & 0 & 0 \\
C^{\star} & 0 & 0
\end{array}\right] \\
& -\operatorname{rank}\left[\begin{array}{ccc}
A & B & C \\
B^{\star} & 0 & 0
\end{array}\right]-\operatorname{rank}\left[\begin{array}{ccc}
A & B & C \\
C^{\star} & 0 & 0
\end{array}\right] \\
\tau_{2}= & 2 \operatorname{rank}\left[\begin{array}{ccc}
A & B & C
\end{array}\right]+\operatorname{rank}\left[\begin{array}{ccc}
A & B & C \\
B^{\star} & 0 & 0
\end{array}\right]-\operatorname{rank}\left[\begin{array}{cc}
A & C \\
B^{\star} & 0
\end{array}\right] \\
\tau_{3}= & \operatorname{rank}\left[\begin{array}{ccc}
A & B & C \\
B^{\star} & 0 & 0
\end{array}\right]+\operatorname{rank}\left[\begin{array}{ccc}
A & B & C \\
C^{\star} & 0 & 0
\end{array}\right] \\
& -\operatorname{rank}\left[\begin{array}{ccc}
A & B & C
\end{array}\right]-\operatorname{rank}\left[\begin{array}{ccc}
A & B & C \\
B^{\star} & 0 & 0 \\
C^{\star} & 0 & 0
\end{array}\right] \\
\tau_{4}= & \operatorname{rank}\left[\begin{array}{ccc}
A & B & C \\
C^{\star} & 0 & 0
\end{array}\right]-\operatorname{rank}\left[\begin{array}{cc}
A & C \\
B^{\star} & 0
\end{array}\right]
\end{aligned}
$$

Moreover,

- if $\mathbb{F}=\mathbb{C}$, then

$$
\begin{gathered}
\left\{\operatorname{rank}\left(\Theta-\Phi\left(W_{B} X W_{B}^{\star}\right) \Phi^{\star}-\Psi\left(W_{C} Y W_{C}^{\star}\right) \Psi^{\star}\right) \mid X= \pm X^{\star} \in \mathbb{C}^{m \times m}\right. \\
\left.Y= \pm Y^{\star} \in \mathbb{C}^{p \times p}\right\} \\
=\left\{s \mid s \text { is integer, } \tau_{1} \leq s \leq 2 \tau_{1}+\tau_{2}+\tau_{3}+\tau_{4}\right\}
\end{gathered}
$$

and for any given integers $i_{+}$and $i_{-}$, there exist matrices $X= \pm X^{\star} \in \mathbb{C}^{m \times m}$ and $Y= \pm Y^{\star} \in \mathbb{C}^{p \times p}$ such that

$$
\left\{\begin{array}{l}
i_{+}=\mathbb{I} n_{+}\left(\Theta-\Phi\left(W_{B} X W_{B}^{\star}\right) \Phi^{\star}-\Psi\left(W_{C} Y W_{C}^{\star}\right) \Psi^{\star}\right), \\
i_{-}=\mathbb{I} n_{-}\left(\Theta-\Phi\left(W_{B} X W_{B}^{\star}\right) \Phi^{\star}-\Psi\left(W_{C} Y W_{C}^{\star}\right) \Psi^{\star}\right)
\end{array}\right.
$$

if and only if

$$
0 \leq i_{+}, \quad 0 \leq i_{-}, \quad \tau_{1} \leq i_{+}+i_{-} \leq 2 \tau_{1}+\tau_{2}+\tau_{3}+\tau_{4} .
$$

- if $\mathbb{F}=\mathbb{R}$ and $\Theta=\Theta^{T}$, then

$$
\begin{gathered}
\left\{\begin{array}{c}
\operatorname{rank}\left(\Theta-\Phi\left(W_{B} X W_{B}^{T}\right) \Phi^{T}-\Psi\left(W_{C} Y W_{C}^{T}\right) \Psi^{T}\right) \mid X \\
=X^{T} \in \mathbb{R}^{m \times m}, Y=Y^{T} \in \mathbb{R}^{p \times p}
\end{array}\right\} \\
\quad=\left\{s \mid s \text { is integer, } \tau_{1} \leq s \leq 2 \tau_{1}+\tau_{2}+\tau_{3}+\tau_{4}\right\},
\end{gathered}
$$

and for any given integers $i_{+}$and $i_{-}$, there exist matrices $X=X^{T} \in \mathbb{R}^{m \times m}$ and $Y=Y^{T} \in \mathbb{R}^{p \times p}$ such that

$$
\left\{\begin{array}{l}
i_{+}=\mathbb{I} n_{+}\left(\Theta-\Phi\left(W_{B} X W_{B}^{T}\right) \Phi^{T}-\Psi\left(W_{C} Y W_{C}^{T}\right) \Psi^{T}\right) \\
i_{-}=\mathbb{I} n_{-}\left(\Theta-\Phi\left(W_{B} X W_{B}^{T}\right) \Phi^{T}-\Psi\left(W_{C} Y W_{C}^{T}\right) \Psi^{T}\right)
\end{array}\right.
$$

if and only if (33) holds.

- if $\mathbb{F}=\mathbb{R}$ and $\Theta=-\Theta^{T}$,

Copyright (c) by SIAM. Unauthorized reproduction of this article is prohibited. 
(i) when $\tau_{1}=0, \tau_{3}=0$, both $\tau_{2}$ and $\tau_{4}$ are all odd, then

$$
\begin{gathered}
\left\{\operatorname{rank}\left(\Theta-\Phi\left(W_{B} X W_{B}^{T}\right) \Phi^{T}-\Psi\left(W_{C} Y W_{C}^{T}\right) \Psi^{T}\right) \mid X\right. \\
\left.=-X^{T} \in \mathbb{R}^{m \times m}, Y=-Y^{T} \in \mathbb{R}^{p \times p}\right\} \\
(36) \quad=\left\{s \mid s \text { is even integer, } \tau_{1} \leq s \leq 2 \tau_{1}+\tau_{2}+\tau_{3}+\tau_{4}-2\right\},
\end{gathered}
$$

and for any given integers $i_{+}$and $i_{-}$, there exist matrices $X=-X^{T} \in$ $\mathbb{R}^{m \times m}$ and $Y=-Y^{T} \in \mathbb{R}^{p \times p}$ satisfying (35) if and only if

$$
i_{+}=i_{-}, \quad \tau_{1} \leq i_{+}+i_{-} \leq 2 \tau_{1}+\tau_{2}+\tau_{3}+\tau_{4}-2
$$

(ii) otherwise,

$$
\begin{aligned}
& \left\{\operatorname{rank}\left(\Theta-\Phi\left(W_{B} X W_{B}^{T}\right) \Phi^{T}-\Psi\left(W_{C} Y W_{C}^{T}\right) \Psi^{T}\right) \mid X\right. \\
& \left.\quad=-X^{T} \in \mathbb{R}^{m \times m}, Y=-Y^{T} \in \mathbb{R}^{p \times p}\right\} \\
& =\left\{s \mid s \text { is even integer, } \tau_{1} \leq s \leq 2 \tau_{1}+\tau_{2}+\tau_{3}+\tau_{4}\right\},
\end{aligned}
$$

and for any given integers $i_{+}$and $i_{-}$, there exist matrices $X=-X^{T} \in$ $\mathbb{R}^{m \times m}$ and $Y=-Y^{T} \in \mathbb{R}^{p \times p}$ satisfying (35) if and only if

$$
i_{+}=i_{-}, \quad \tau_{1} \leq i_{+}+i_{-} \leq 2 \tau_{1}+\tau_{2}+\tau_{3}+\tau_{4} .
$$

Proof. Note that $\Theta= \pm \Theta^{\star}$,

$$
\Theta=\left[\begin{array}{cc}
\mathcal{A}_{22} & \mathcal{A}_{23} \\
\pm \mathcal{A}_{23}^{\star} & \tilde{A}_{33}
\end{array}\right], \quad \Phi=\left[\begin{array}{cc}
0 & \mathcal{B}_{22} \\
0 & 0
\end{array}\right], \quad \Psi=\left[\begin{array}{cc}
\mathcal{C}_{21} & 0 \\
0 & \tilde{C}_{32}
\end{array}\right],
$$

and $\mathcal{B}_{22} \in \mathbb{F}^{\mu_{2} \times \mu_{2}}$ and $\tilde{C}_{32} \in \mathbb{F}^{\mu_{3} \times \mu_{3}}$ are nonsingular, so we can construct the form (29) by the following steps:

Step 1: Compute unitary matrices $P_{1} \in \mathbb{F}^{\mu_{2} \times \mu_{2}}$ and $Q \in \mathbb{F}^{p \times p}$ such that

$$
\left.P_{1} \mathcal{C}_{21} Q^{\star}=\left[\begin{array}{cc}
p-\mu_{3}-\tau_{3} & \tau_{3} \\
0 & 0 \\
0 & \Psi_{32}
\end{array}\right]\right\} \mu_{2}-\tau_{3}, \quad \operatorname{rank}\left(\Psi_{32}\right)=\tau_{3} .
$$

Denote

$$
\left.\left[\begin{array}{ll}
P_{1} & \\
& I
\end{array}\right] \Theta\left[\begin{array}{ll}
P_{1} & \\
& I
\end{array}\right]^{\star}=\left[\begin{array}{ccc}
\mu_{2}-\tau_{3} & \tau_{3} & \mu_{3} \\
\Theta_{11}^{(1)} & \Theta_{12}^{(1)} & \Theta_{13}^{(1)} \\
\pm\left(\Theta_{12}^{(1)}\right)^{\star} & \Theta_{22}^{(1)} & \Theta_{23}^{(1)} \\
\pm\left(\Theta_{13}^{(1)}\right)^{\star} & \pm\left(\Theta_{23}^{(1)}\right)^{\star} & \Theta_{33}^{(1)}
\end{array}\right]\right\} \mu_{2}-\tau_{3}
$$

Step 2: Compute the SVD of $\Theta_{13}^{(1)}$ to get unitary matrices $P_{2} \in \mathbb{F}^{\left(\mu_{2}-\tau_{3}\right) \times\left(\mu_{2}-\tau_{2}\right)}$ and $P_{3} \in \mathbb{F}^{\mu_{3} \times \mu_{3}}$ such that

$$
\left.P_{2} \Theta_{13}^{(1)} P_{3}^{\star}=\left[\begin{array}{cc}
\tau_{4} & \tau_{1} \\
0 & \Sigma \\
0 & 0
\end{array}\right]\right\} \tau_{1}
$$

Copyright (c) by SIAM. Unauthorized reproduction of this article is prohibited. 
where $\Sigma=\left[\begin{array}{ccc}\sigma_{1} & & \\ & \ddots & \\ & & \sigma_{\tau_{1}}\end{array}\right]$ is nonsingular. Define
$P=\left[\begin{array}{lll}P_{2} & & \\ & I & \\ & & P_{3}\end{array}\right]\left[\begin{array}{ll}P_{1} & \\ & I\end{array}\right]$.

Then $\left(P \Theta P^{\star}, P \Phi, P \Psi Q^{\star}\right)$ is of the form (29) with

$$
\begin{gathered}
\operatorname{rank}\left[\begin{array}{c}
\Phi_{12} \\
\Phi_{22} \\
\Phi_{32}
\end{array}\right]=\operatorname{rank}\left(\left[\begin{array}{cc}
P_{2} & \\
& I
\end{array}\right] P_{1} \mathcal{B}_{22}\right)=\operatorname{rank}\left(\mathcal{B}_{22}\right)=\mu_{2}=\tau_{1}+\tau_{2}+\tau_{3}, \\
\operatorname{rank}\left[\begin{array}{l}
\Psi_{43} \\
\Psi_{53}
\end{array}\right]=\operatorname{rank}\left(P_{3} \tilde{C}_{32}\right)=\operatorname{rank}\left(\tilde{C}_{32}\right)=\mu_{3}=\tau_{4}+\tau_{1},
\end{gathered}
$$

and

$$
\Theta_{i, i}= \pm \Theta_{i, i}^{\star}, i=1, \ldots, 5\left(\text { since } \Theta= \pm \Theta^{\star}\right) \text {. }
$$

Clearly, we have

$$
\begin{aligned}
& \left.\tau_{1}+\tau_{2}+\tau_{3}=\operatorname{rank} \Phi\right), \\
& \tau_{1}+\tau_{3}+\tau_{4}=\operatorname{rank}(\Psi), \\
& 3 \tau_{1}+\tau_{2}+2 \tau_{3}+\tau_{4}=\operatorname{rank}\left[\begin{array}{cc}
\Theta & \Phi \\
\Psi^{\star} & 0
\end{array}\right], \\
& 2 \tau_{1}+\tau_{2}+\tau_{3}+\tau_{4}=\mu_{2}+\mu_{3},
\end{aligned}
$$

which together with $(24)$ and $(26)$ give (30). Furthermore, $W_{B}, W_{C},\left[\begin{array}{l}\Phi_{12} \\ \Phi_{22} \\ \Phi_{32}\end{array}\right]$, and $\left[\begin{array}{cc}\Psi_{32} & 0 \\ 0 & \Psi_{43} \\ 0 & \Psi_{53}\end{array}\right]$ are all nonsingular, for any $X= \pm X^{\star} \in \mathbb{F}^{m \times m}$ and $Y= \pm Y^{\star} \in \mathbb{F}^{p \times p}$, we can let

$$
\left[\begin{array}{ll}
0 & \Phi_{12} \\
0 & \Phi_{22} \\
0 & \Phi_{32}
\end{array}\right] W_{B} X W_{B}^{\star}\left[\begin{array}{ll}
0 & \Phi_{12} \\
0 & \Phi_{22} \\
0 & \Phi_{32}
\end{array}\right]^{\star}=\left[\begin{array}{ccc}
\Theta_{11} & \Theta_{12} & \Theta_{13} \\
\pm \Theta_{12}^{\star} & \Theta_{22} & \Theta_{23} \\
\pm \Theta_{13}^{\star} & \pm \Theta_{23}^{\star} & \Theta_{33}
\end{array}\right]-\left[\begin{array}{ccc}
Z_{11} & Z_{12} & Z_{13} \\
\pm Z_{12}^{\star} & Z_{22} & Z_{23} \\
\pm Z_{13}^{\star} & \pm Z_{23}^{\star} & Z_{33}
\end{array}\right]
$$

and

$$
\begin{gathered}
{\left[\begin{array}{ccc}
0 & \Psi_{32} & 0 \\
0 & 0 & \Psi_{43} \\
0 & 0 & \Psi_{53}
\end{array}\right] Q W_{C} Y W_{C}^{\star} Q^{\star}\left[\begin{array}{ccc}
0 & \Psi_{32} & 0 \\
0 & 0 & \Psi_{43} \\
0 & 0 & \Psi_{53}
\end{array}\right]^{\star}} \\
=\left[\begin{array}{ccc}
0 & \Theta_{34} & \Theta_{35} \\
\pm \Theta_{34}^{\star} & \Theta_{44} & \Theta_{45} \\
\pm \Theta_{35}^{\star} & \pm \Theta_{45}^{\star} & \Theta_{55}
\end{array}\right]-\left[\begin{array}{ccc}
\mathcal{Z}_{33} & Z_{34} & Z_{35} \\
\pm Z_{34}^{\star} & Z_{44} & Z_{45} \\
\pm Z_{35}^{\star} & Z_{45}^{\star} & Z_{55}
\end{array}\right],
\end{gathered}
$$

where $Z_{i i}= \pm Z_{i i}^{\star} \in \mathbb{F}^{\tau_{i} \times \tau_{i}}(i=1, \ldots, 4), \mathcal{Z}_{33}= \pm \mathcal{Z}_{33}^{\star} \in \mathbb{F}^{\tau_{3} \times \tau_{3}}$, and $Z_{55}= \pm Z_{55}^{\star} \in$ $\mathbb{F}^{\tau_{1} \times \tau_{1}} ;$ consequently,

$$
P\left(\Theta-\Phi\left(W_{B} X W_{B}^{\star}\right) \Phi^{\star}-\Psi\left(W_{C} Y W_{C}^{\star}\right) \Psi^{\star}\right) P^{\star}=\left[\begin{array}{ccccc}
Z_{11} & Z_{12} & Z_{13} & 0 & \Sigma \\
\pm Z_{12}^{\star} & Z_{22} & Z_{23} & 0 & 0 \\
\pm Z_{13}^{\star} & \pm Z_{23}^{\star} & Z_{33}+\mathcal{Z}_{33} & Z_{34} & Z_{35} \\
0 & 0 & \pm Z_{34}^{\star} & Z_{44} & Z_{45} \\
\pm \Sigma^{\star} & 0 & \pm Z_{35}^{\star} & Z_{45}^{\star} & Z_{55}
\end{array}\right] .
$$

Hence, by Lemmas 6 and 7, (31)-(39) follows. 
We are now ready to provide the alternative proof of Theorem 1.

Proof of Theorem 1. Theorem 1 and the desired matrices $X$ and $Y$ involved follow directly from Lemmas $9-11$ and their proofs.

4. Proof of Theorem 3. We prove Theorem 3 in this section. It is obvious that for any $X \in \mathbb{F}^{m \times p}$

$$
\mathbb{I n}\left(\left[\begin{array}{ccccc}
A & B & C & 0 & 0 \\
\pm B^{*} & 0 & 0 & I_{m} & 0 \\
\pm C^{*} & 0 & 0 & 0 & I_{p} \\
0 & \pm I_{m} & 0 & 0 & X \\
0 & 0 & \pm I_{p} & \pm X^{*} & 0
\end{array}\right]\right)=\mathbb{I} n\left(A-B X C^{\star} \pm C X^{\star} B^{\star}\right)+(m+p, m+p, 0) ;
$$

thus, the inertia characterization (13) in Theorem 3 follow directly from [7, Theorem 1.1]. Although [7, Theorem 1.1] concerns the complex self-adjoint case, it is easy to check that their results are also valid in the real symmetric case and the complex skew-adjoint case. It remains to show the results on all admissible ranks and the real skew-symmetric case. Since Lemma 6 holds, here we need only to prove (12), (14), and (15). For this purpose, we need some supporting results as follows.

Lemma 12. Given $\Sigma_{1}= \pm \Sigma_{1}^{\star} \in \mathbb{F}^{\nu_{1} \times \nu_{1}}, \Sigma_{2}= \pm \Sigma_{2}^{\star} \in \mathbb{F}^{\nu_{2} \times \nu_{2}}$, and $\Sigma_{1}$ and $\Sigma_{2}$ are nonsingular.

- If $\mathbb{F}=\mathbb{C}$, or $\mathbb{F}=\mathbb{R}$ and $\Sigma_{i}=\Sigma_{i}^{T}(i=1,2)$, then

$$
\begin{aligned}
& \left\{\operatorname{rank}\left[\begin{array}{cc}
\Sigma_{1} & \mathcal{Y} \\
\pm \mathcal{Y}^{\star} & \Sigma_{2}
\end{array}\right] \mid \mathcal{Y} \in \mathbb{F}^{\nu_{1} \times \nu_{2}}\right\} \\
= & \left\{s \text { is integer and } \max \left\{\mathcal{I}_{+}\left(\Sigma_{1}\right), \mathcal{I}_{+}\left(\Sigma_{2}\right)\right\}\right. \\
& \left.\quad+\max \left\{\mathcal{I}_{-}\left(\Sigma_{1}\right), \mathcal{I}_{-}\left(\Sigma_{2}\right)\right\} \leq s \leq \nu_{1}+\nu_{2}\right\} ;
\end{aligned}
$$

- If $\mathbb{F}=\mathbb{R}, \Sigma_{i}=-\Sigma_{i}^{T}(i=1,2)$, then

$$
\begin{aligned}
& \left\{\operatorname{rank}\left[\begin{array}{cc}
\Sigma_{1} & \mathcal{Y} \\
-\mathcal{Y}^{T} & \Sigma_{2}
\end{array}\right] \mid \mathcal{Y} \in \mathbb{R}^{\nu_{1} \times \nu_{2}}\right\} \\
= & \left\{s \text { is even integer and } \max \left\{\nu_{1}, \nu_{2}\right\} \leq s \leq \nu_{1}+\nu_{2}\right\} .
\end{aligned}
$$

Proof. The proof of Lemma 12 and the construction of the desired matrix $\mathcal{Y}$ are straightforward and thus are omitted here.

Lemma 13. Given $\mathcal{F}= \pm \mathcal{F}^{\star} \in \mathbb{F}^{k_{1} \times k_{1}}$ and $\mathcal{G}= \pm \mathcal{G}^{\star} \in \mathbb{F}^{k_{2} \times k_{2}}$. Let

$$
\mathcal{Z}=\left[\begin{array}{cc}
\mathcal{F} & \mathcal{Z}_{2} \\
\pm \mathcal{Z}_{2}^{\star} & \mathcal{G}
\end{array}\right]= \pm \mathcal{Z}^{\star}
$$

Denote

$$
\begin{aligned}
\mathcal{K}_{\text {min }} & =\max \left\{\mathcal{I}_{+}(\mathcal{F}), \mathcal{I}_{+}(\mathcal{G})\right\}+\max \left\{\mathcal{I}_{-}(\mathcal{F}), \mathcal{I}_{-}(\mathcal{G})\right\}, \\
\mathcal{K}_{\text {max }} & =\min \left\{2 k_{1}+\operatorname{rank}(\mathcal{G}), 2 k_{2}+\operatorname{rank}(\mathcal{F}), k_{1}+k_{2}\right\} .
\end{aligned}
$$

- If $\mathbb{F}=\mathbb{C}$, or $\mathbb{F}=\mathbb{R}, \mathcal{F}=\mathcal{F}^{T}$, and $\mathcal{G}=\mathcal{G}^{T}$, then

$$
\{\operatorname{rank}(\mathcal{Z})\}=\left\{s \text { is integer and } \mathcal{K}_{\min } \leq s \leq \mathcal{K}_{\max }\right\}
$$

- If $\mathbb{F}=\mathbb{R}, \mathcal{F}=-\mathcal{F}^{T}$, and $\mathcal{G}=-\mathcal{G}^{T}$, then

$$
\{\operatorname{rank}(\mathcal{Z})\}=\left\{s \text { is even integer and } \max \{\operatorname{rank}(\mathcal{F}), \operatorname{rank}(\mathcal{G})\} \leq s \leq \mathcal{K}_{\text {max }}\right\} .
$$

Copyright $@$ ( ) by SIAM. Unauthorized reproduction of this article is prohibited. 
Proof. We can assume by computing the real Schur forms of $\mathcal{F}$ and $\mathcal{G}$ that

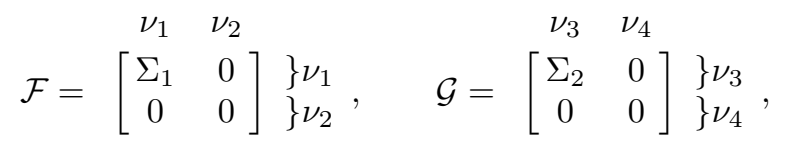

where

$$
\nu_{1}=\operatorname{rank}(\mathcal{F}), \quad \nu_{3}=\operatorname{rank}(\mathcal{G}), \quad \nu_{1}+\nu_{2}=k_{1}, \quad \nu_{3}+\nu_{4}=k_{2},
$$

$\Sigma_{1}$ and $\Sigma_{2}$ are nonsingular, and

- $\Sigma_{1}= \pm \Sigma_{1}^{\star}$ and $\Sigma_{2}= \pm \Sigma_{2}^{\star}$ are diagonal if $\mathbb{F}=\mathbb{C}$, or $\mathbb{F}=\mathbb{R}, \mathcal{F}=\mathcal{F}^{T}$, and $\mathcal{G}=\mathcal{G}^{T}$

- $\Sigma_{1}=-\Sigma^{T}$ and $\Sigma_{2}=-\Sigma^{T}$ are all block diagonal with all diagonal blocks $2 \times 2$ if $\mathbb{F}=\mathbb{R}, \mathcal{F}=-\mathcal{F}^{T}$, and $\mathcal{G}=-\mathcal{G}^{T}$.

Denote

$$
\left.\mathcal{Z}_{2}=\left[\begin{array}{cc}
\nu_{3} & \nu_{4} \\
\mathcal{Z}_{13} & \mathcal{Z}_{14} \\
\mathcal{Z}_{23} & \mathcal{Z}_{24}
\end{array}\right]\right\} \nu_{1}
$$

Then $\mathcal{Z}$ is of the form

$$
\mathcal{Z}=\left[\begin{array}{cccc}
\nu_{1} & \nu_{2} & \nu_{3} & \nu_{4} \\
\Sigma_{1} & 0 & \mathcal{Z}_{13} & \mathcal{Z}_{14} \\
0 & 0 & \mathcal{Z}_{23} & \mathcal{Z}_{24} \\
\pm \mathcal{Z}_{13}^{\star} & \pm \mathcal{Z}_{23}^{\star} & \Sigma_{2} & 0 \\
\pm \mathcal{Z}_{14}^{\star} & \pm \mathcal{Z}_{24}^{\star} & 0 & 0
\end{array}\right] \begin{gathered}
\} \nu_{1} \\
\} \nu_{2} \\
\} \nu_{3} \\
\nu_{4}
\end{gathered}= \pm \mathcal{Z}^{\star} \in \mathbb{F}^{\sum_{i=1}^{4} \nu_{i} \times \sum_{i=1}^{4} \nu_{i}}
$$

Obviously, for any $\mathcal{Z}$ we have by using Lemma 12 that

$$
\begin{aligned}
\operatorname{rank}(\mathcal{Z}) \geq \operatorname{rank}\left[\begin{array}{cc}
\Sigma_{1} & \mathcal{Z}_{13} \\
\pm \mathcal{Z}_{13}^{\star} & \Sigma_{2}
\end{array}\right] \\
\quad \geq\left\{\begin{array}{cc}
\max \left\{\mathcal{I}_{+}\left(\Sigma_{1}\right), \mathcal{I}_{+}\left(\Sigma_{2}\right)\right\}+\max \left\{\mathcal{I}_{-}\left(\Sigma_{1}\right), \mathcal{I}_{-}\left(\Sigma_{2}\right)\right\} \\
=\mathcal{K}_{\min }, & \text { if } \mathbb{F}=\mathbb{C}, \text { or } \mathbb{F}=\mathbb{R}, \mathcal{F}=\mathcal{F}^{T}, \mathcal{G}=\mathcal{G}^{T}, \\
\max \left\{\nu_{1}, \nu_{3}\right\}=\max \{\operatorname{rank}(\mathcal{F}), \operatorname{rank}(\mathcal{G})\}, & \text { if } \mathbb{F}=\mathbb{R}, \mathcal{F}=-\mathcal{F}^{T}, \mathcal{G}=-\mathcal{G}^{T} .
\end{array}\right.
\end{aligned}
$$

On the other hand, we can assume without loss of generality that

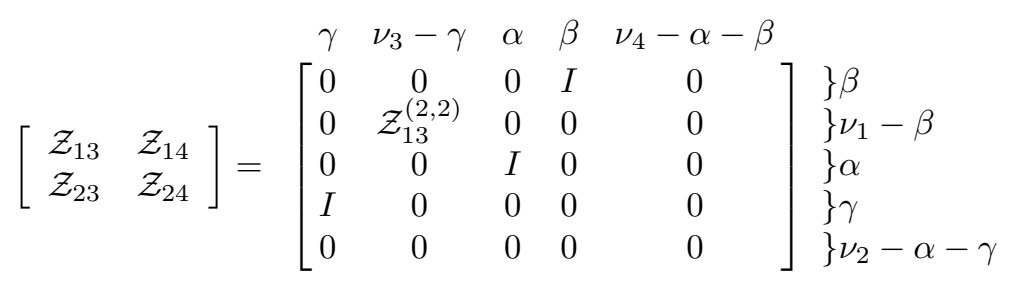

and

$$
\left.\left.\Sigma_{1}=\left[\begin{array}{cl}
\beta & \nu_{1}-\beta \\
\Sigma_{1}^{(1,1)} & \Sigma_{1}^{(1,2)} \\
\pm\left(\Sigma_{1}^{(1,2)}\right)^{\star} & \Sigma_{1}^{(2,2)}
\end{array}\right]\right\} \beta \quad \begin{array}{cc}
\gamma & \nu_{3}-\gamma \\
\} \nu_{1}-\beta
\end{array}, \quad \Sigma_{2}=\left[\begin{array}{cc}
\Sigma_{2}^{(1,1)} & \Sigma_{2}^{(1,2)} \\
\pm\left(\Sigma_{2}^{(1,2)}\right)^{\star} & \Sigma_{2}^{(2,2)}
\end{array}\right]\right\} \gamma
$$

Copyright (c) by SIAM. Unauthorized reproduction of this article is prohibited. 
where

$$
\alpha \leq \min \left\{\nu_{2}, \nu_{4}\right\}, \quad \beta \leq \min \left\{\nu_{1}, \nu_{4}-\alpha\right\}, \quad \gamma \leq \min \left\{\nu_{2}-\alpha, \nu_{3}\right\} .
$$

Consequently, we obtain

$$
\begin{aligned}
\operatorname{rank}(\mathcal{Z}) & =2(\alpha+\beta+\gamma)+\operatorname{rank}\left[\begin{array}{cc}
\Sigma_{1}^{(2,2)} & \mathcal{Z}_{13}^{(2,2)} \\
\pm\left(\mathcal{Z}_{13}^{(2,2)}\right)^{\star} & \Sigma_{2}^{(2,2)}
\end{array}\right] \\
& \leq 2(\alpha+\beta+\gamma)+\left[\left(\nu_{1}-\beta\right)+\left(\nu_{3}-\gamma\right)\right]=\nu_{1}+\nu_{3}+2 \alpha+\beta+\gamma \\
& \leq \mathcal{K}_{\text {max }}
\end{aligned}
$$

In addition,

- when $\mathbb{F}=\mathbb{C}$, or $\mathbb{F}=\mathbb{R}, \mathcal{F}=\mathcal{F}^{T}, \mathcal{G}=\mathcal{G}^{T}$, a simple calculation with Lemma 12 yields that

$$
\begin{aligned}
& \left\{\operatorname{rank}(\mathcal{Z}) \mid\left[\begin{array}{ll}
\mathcal{Z}_{13} & \mathcal{Z}_{14} \\
\mathcal{Z}_{23} & \mathcal{Z}_{24}
\end{array}\right] \text { is of the form }(43)\right\} \\
= & \left\{s \mid s \text { is integer and } \mathcal{K}_{\min } \leq s \leq \nu_{1}+\nu_{3}\right\} \\
& \cup\left\{\nu_{1}+\nu_{3}+2 \alpha+\beta+\gamma \mid \alpha \leq \min \left\{\nu_{2}, \nu_{4}\right\},\right. \\
& \left.\beta \leq \min \left\{\nu_{1}, \nu_{4}-\alpha\right\}, \gamma \leq \min \left\{\nu_{2}-\alpha, \nu_{3}\right\}\right\} \\
= & \left\{s \mid s \text { is integer and } \mathcal{K}_{\min } \leq s \leq \mathcal{K}_{\max }\right\}
\end{aligned}
$$

- similarly, when $\mathbb{F}=\mathbb{R}, \Sigma_{i}=-\Sigma_{i}^{T}(i=1,2)$, a simple calculation with Lemma 12 again gives that

$$
\begin{aligned}
& \left\{\operatorname{rank}(\mathcal{Z}) \mid\left[\begin{array}{ll}
\mathcal{Z}_{13} & \mathcal{Z}_{14} \\
\mathcal{Z}_{23} & \mathcal{Z}_{24}
\end{array}\right] \text { is of the form }(43), \beta \text { and } \gamma \text { are even }\right\} \\
= & \left\{s \mid s \text { is even integer and } \max \left\{\nu_{1}, \nu_{3}\right\} \leq s \leq \nu_{1}+\nu_{3}\right\} \\
& \cup\left\{\nu_{1}+\nu_{3}+2 \alpha+\beta+\gamma \mid \alpha \leq \min \left\{\nu_{2}, \nu_{4}\right\}, \beta \leq \min \left\{\nu_{1}, \nu_{4}-\alpha\right\},\right. \\
\left.\gamma \leq \min \left\{\nu_{2}-\alpha, \nu_{3}\right\}, \beta \text { and } \gamma \text { are even }\right\} & \\
= & \left\{s \mid s \text { is even integer and } \max \left\{\nu_{1}, \nu_{3}\right\} \leq s \leq \mathcal{K}_{\max }\right\} .
\end{aligned}
$$

Hence, Lemma 13 follows from (42) and (44)-(46).

Lemma 14. Given $\mathcal{F}= \pm \mathcal{F}^{\star} \in \mathbb{F}^{k_{1} \times k_{1}}$ and $\mathcal{G}= \pm \mathcal{G}^{\star} \in \mathbb{F}^{k_{2} \times k_{2}}$. Let

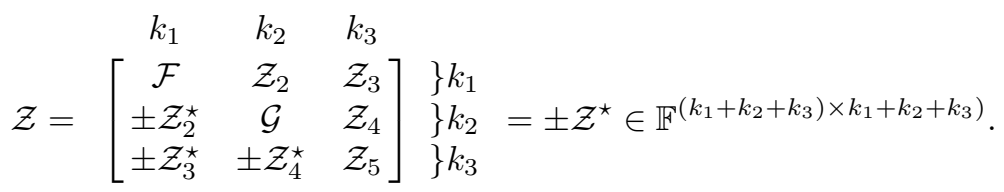

Denote

$$
\begin{aligned}
& \mathcal{K}_{\text {min }}=\max \left\{\mathcal{I}_{+}(\mathcal{F}), \mathcal{I}_{+}(\mathcal{G})\right\}+\max \left\{\mathcal{I}_{-}(\mathcal{F}), \mathcal{I}_{-}(\mathcal{G})\right\}, \\
& \hat{\mathcal{K}}_{\text {max }}=\min \left\{2 k_{1}+2 k_{3}+\operatorname{rank}(\mathcal{G}), 2 k_{2}+2 k_{3}+\operatorname{rank}(\mathcal{F}), k_{1}+k_{2}+k_{3}\right\} .
\end{aligned}
$$

- If $\mathbb{F}=\mathbb{C}$, or $\mathbb{F}=\mathbb{R}, \mathcal{F}=\mathcal{F}^{T}$, and $\mathcal{G}=\mathcal{G}^{T}$, then

$$
\{\operatorname{rank}(\mathcal{Z})\}=\left\{s \text { is integer and } \mathcal{K}_{\text {min }} \leq s \leq \hat{\mathcal{K}}_{\text {max }}\right\} ;
$$

Copyright $@$ by SIAM. Unauthorized reproduction of this article is prohibited. 
- If $\mathbb{F}=\mathbb{R}, \mathcal{F}=-\mathcal{F}^{T}$ and $\mathcal{G}=-\mathcal{G}^{T}$, then

$$
\{\operatorname{rank}(\mathcal{Z})\}=\left\{s \text { is even integer and } \max \{\operatorname{rank}(\mathcal{F}), \operatorname{rank}(\mathcal{G})\} \leq s \leq \hat{\mathcal{K}}_{\text {max }}\right\} .
$$

Proof. Obviously, we have by using Lemma 13 that

$$
\operatorname{rank}(\mathcal{Z}) \geq \operatorname{rank}\left[\begin{array}{cc}
\mathcal{F} & \mathcal{Z}_{2} \\
\pm \mathcal{Z}_{2}^{\star} & \mathcal{G}
\end{array}\right] \geq \mathcal{K}_{\text {min }} \quad \text { for all } \mathcal{Z}
$$

Furthermore, for a $\mathcal{Z}$, let unitary matrices $\mathcal{U} \in \mathbb{F}^{\left(k_{1}+k_{2}\right) \times\left(k_{1}+k_{2}\right)}$ and $\mathcal{W} \in \mathbb{F}^{k_{3} \times k_{3}}$ be such that

$$
\begin{aligned}
& \beta \quad k_{1}+k_{2}-\beta \quad \gamma \quad k_{3}-\gamma \\
& \left.\mathcal{U}^{\star}\left[\begin{array}{cc|c}
\mathcal{F} & \mathcal{Z}_{2} & \mathcal{Z}_{3} \\
\pm \mathcal{Z}_{2}^{\star} & \mathcal{G} & \mathcal{Z}_{4}
\end{array}\right]\left[\begin{array}{cc}
\mathcal{U} & \\
& \mathcal{W}
\end{array}\right]=\left[\begin{array}{cccc}
\Lambda & 0 & \hat{\mathcal{Z}}_{3}^{(1)} & \hat{\mathcal{Z}}_{3}^{(2)} \\
0 & 0 & \hat{\mathcal{Z}}_{4} & 0
\end{array}\right]\right\} k_{1}+k_{2}-\beta,
\end{aligned}
$$

where

$$
\operatorname{rank}(\Lambda)=\operatorname{rank}\left[\begin{array}{cc}
\mathcal{F} & \mathcal{Z}_{2} \\
\pm \mathcal{Z}_{2}^{\star} & \mathcal{G}
\end{array}\right]=\beta, \quad \operatorname{rank}\left(\hat{\mathcal{Z}}_{4}\right)=\gamma
$$

By Lemma 13, we know

$$
\beta \leq \min \left\{2 k_{1}+\operatorname{rank}(\mathcal{G}), 2 k_{2}+\operatorname{rank}(\mathcal{F}), k_{1}+k_{2}\right\} .
$$

We also have

$$
\gamma \leq \min \left\{k_{3}, k_{1}+k_{2}-\beta\right\}
$$

Thus, we get

$$
\begin{aligned}
\operatorname{rank}(\mathcal{Z}) & \leq 2 \gamma+\beta+\left(k_{3}-\gamma\right)=\beta+\gamma+k_{3} \leq \min \left\{k_{3}+\beta, k_{1}+k_{2}\right\}+k_{3} \\
& \leq \min \left\{k_{3}+\min \left\{2 k_{1}+\operatorname{rank}(\mathcal{G}), 2 k_{2}+\operatorname{rank}(\mathcal{F}), k_{1}+k_{2}\right\}, k_{1}+k_{2}\right\}+k_{3} \\
& \leq \min \left\{2 k_{1}+k_{3}+\operatorname{rank}(\mathcal{G}), 2 k_{2}+k_{3}+\operatorname{rank}(\mathcal{F}), k_{1}+k_{2}\right\}+k_{3} \\
& =\hat{\mathcal{K}}_{\text {max }} .
\end{aligned}
$$

Furthermore, we can assume by computing the real Schur forms of $\mathcal{F}$ and $\mathcal{G}$ that $\mathcal{F}$ and $\mathcal{G}$ are of the forms in (40). Take

$$
\left\{\begin{array}{l}
\hat{\mathcal{Z}}_{5}=\left[\begin{array}{cc}
I_{\alpha} & 0 \\
0 & 0
\end{array}\right], \\
\hat{\mathcal{Z}}_{3}=\left[\begin{array}{cc}
0 & 0 \\
I_{\beta} & 0 \\
0 & 0
\end{array}\right]{ }_{\} \nu_{2}-\beta}^{\} \nu_{1}}, \quad \hat{\mathcal{Z}}_{4}=\left[\begin{array}{cc}
0 & 0 \\
I_{\gamma} & 0 \\
0 & 0
\end{array}\right] \begin{array}{l}
\} \nu_{3} \\
\} \nu_{4}-\gamma
\end{array}
\end{array}\right.
$$

$$
\text { if } \mathbb{F}=\mathbb{C} \text {, or } \mathbb{F}=\mathbb{R}, \mathcal{F}=\mathcal{F}^{T}, \mathcal{G}=\mathcal{G}^{T} \text {, }
$$

and

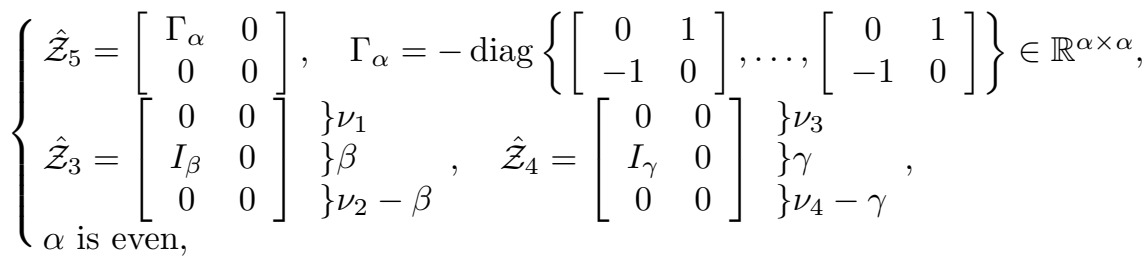

$$
\begin{aligned}
& \text { if } \mathbb{F}=\mathbb{R}, \mathcal{F}=-\mathcal{F}^{T}, \mathcal{G}=-\mathcal{G}^{T} .
\end{aligned}
$$

Copyright (c) by SIAM. Unauthorized reproduction of this article is prohibited. 
Then we have using Lemma 13 that

$$
\begin{aligned}
& \left\{\operatorname{rank}\left[\begin{array}{ccc}
\mathcal{F} & \mathcal{Z}_{2} & \hat{\mathcal{Z}}_{3} \\
\pm \mathcal{Z}_{2}^{\star} & \mathcal{G} & \hat{\mathcal{Z}}_{4} \\
\pm \hat{\mathcal{Z}}_{3}^{\star} & \pm \hat{\mathcal{Z}}_{4}^{\star} & \hat{\mathcal{Z}}_{5}
\end{array}\right] \mid \mathcal{Z}_{2} \in \mathbb{F}^{k_{1} \times k_{2}}, \alpha \leq k_{3}, \beta \leq \nu_{2}, \gamma \leq \nu_{4}, \min \{\beta, \gamma\} \leq \alpha\right\} \\
= & \left\{\begin{array}{c}
\left\{s \mid s \text { is integer and } \mathcal{K}_{\text {min }} \leq s \leq \hat{\mathcal{K}}_{\text {max }}\right\} \quad \text { if } \mathbb{F}=\mathbb{C}, \text { or } \mathbb{F}=\mathbb{R}, \mathcal{F}=\mathcal{F}^{T}, \mathcal{G}=\mathcal{G}^{T}, \\
\left\{s \mid s \text { is even integer and } \max \{\operatorname{rank}(\mathcal{F}), \operatorname{rank}(\mathcal{G})\} \leq s \leq \hat{\mathcal{K}}_{\text {max }}\right\} \\
\text { if } \mathbb{F}=\mathbb{R}, \mathcal{F}=-\mathcal{F}^{T}, \mathcal{G}=-\mathcal{G}^{T} .
\end{array}\right.
\end{aligned}
$$

As a result, we obtain

$$
\left\{\begin{array}{c}
\left\{s \mid s \text { is integer and } \mathcal{K}_{\text {min }} \leq s \leq \mathcal{K}_{\text {max }}\right\} \subset\{\operatorname{rank}(\mathcal{Z})\} \\
\text { if } \mathbb{F}=\mathbb{C}, \text { or } \mathbb{F}=\mathbb{R}, \mathcal{F}=\mathcal{F}^{T}, \mathcal{G}=\mathcal{G}^{T} \\
\left\{s \mid s \text { is even integer and } \max \{\operatorname{rank}(\mathcal{F}), \operatorname{rank}(\mathcal{G})\} \leq s \leq \hat{\mathcal{K}}_{\max }\right\} \subset\{\operatorname{rank}(\mathcal{Z})\}, \\
\text { if } \mathbb{F}=\mathbb{R}, \mathcal{F}=-\mathcal{F}^{T}, \mathcal{G}=-\mathcal{G}^{T} .
\end{array}\right.
$$

Therefore, Lemma 14 follows directly from (47)-(49).

Again it should be pointed out that a simple construction method such that $\mathcal{Z}$ achieves any given admissible rank is embedded in the proofs of Lemmas 12-14.

We are now ready to characterize all admissible ranks of $A-B X C^{\star} \pm C X^{\star} B^{\star}$ in Theorem 3.

Proof of rank characterizations in Theorem 3. Let the factorizations in Lemmas 911 have been determined. Then we have

$$
\begin{aligned}
& \operatorname{rank}\left(A-B X C^{\star} \pm C X^{\star} B^{\star}\right) \\
= & 2 \mu_{1}+\mu_{4}+\operatorname{rank}\left(\Theta-\Phi\left(W_{B} X W_{C}^{\star}\right) \Psi^{\star} \pm \Psi\left(W_{B} X W_{C}^{\star}\right)^{\star} \Phi^{\star}\right) \\
= & \left.2 \mu_{1}+\mu_{4}+\operatorname{rank}\left[P\left(\Theta-\Phi\left(W_{B} X W_{C}^{\star} Q^{\star}\right)\right) Q \Psi^{\star} \pm \Psi Q^{\star}\left(W_{B} X W_{C}^{\star} Q^{\star}\right)^{\star} \Phi^{\star}\right] P^{\star}\right] \\
= & 2 \mu_{1}+\mu_{4}+\operatorname{rank}\left[\begin{array}{ccc}
\mathcal{F} & \mathcal{Z}_{2} & \mathcal{Z}_{3} \\
\pm \mathcal{Z}_{2}^{\star} & \mathcal{Z}_{5} & \mathcal{Z}_{4} \\
\pm \mathcal{Z}_{3}^{\star} & \pm \mathcal{Z}_{4}^{\star} & \mathcal{G}
\end{array}\right],
\end{aligned}
$$

where

$$
\begin{gathered}
\mathcal{F}=\left[\begin{array}{cc}
\Theta_{11} & \Theta_{12} \\
\pm \Theta_{12}^{\star} & \Theta_{22}
\end{array}\right], \quad \mathcal{G}=\left[\begin{array}{cc}
\Theta_{44} & \Theta_{45} \\
\pm \Theta_{45}^{\star} & \Theta_{55}
\end{array}\right] \\
{\left[\begin{array}{ll}
\mathcal{Z}_{2} & \mathcal{Z}_{3}
\end{array}\right]=\left[\begin{array}{ccc}
\Theta_{13} & 0 & \Sigma \\
\Theta_{23} & 0 & 0
\end{array}\right]-\left[\begin{array}{cc}
0 & \Phi_{12} \\
0 & \Phi_{22}
\end{array}\right]\left(W_{B} X W_{C}^{\star} Q^{\star}\right)\left[\begin{array}{ccc}
0 & \Psi_{32} & 0 \\
0 & 0 & \Psi_{43} \\
0 & 0 & \Psi_{53}
\end{array}\right],} \\
\mathcal{Z}_{4}=\left[\begin{array}{ll}
\Theta_{34} & \Theta_{35}
\end{array}\right]-\left[\begin{array}{ll}
0 & \Phi_{32}
\end{array}\right]\left(W_{B} X W_{C}^{\star} Q^{\star}\right)\left[\begin{array}{ccc}
0 & 0 & \Psi_{43} \\
0 & 0 & \Psi_{53}
\end{array}\right]
\end{gathered}
$$

and

$\mathcal{Z}_{5}=\Theta_{33}-\left[\begin{array}{ll}0 & \Phi_{32}\end{array}\right]\left(W_{B} X W_{C}^{\star} Q^{\star}\right)\left[\begin{array}{lll}0 & \Psi_{32} & 0\end{array}\right]^{\star} \pm\left[\begin{array}{lll}0 & \Psi_{32} & 0\end{array}\right]\left(W_{B} X W_{C}^{\star} Q^{\star}\right)^{\star}\left[\begin{array}{ll}0 & \Phi_{32}\end{array}\right]^{\star}$. 
Thus, we obtain by using Lemma 14 that

- if $\mathbb{F}=\mathbb{C}$, or $\mathbb{F}=\mathbb{R}$ and $A=A^{T}$, then

$$
\begin{aligned}
& \left\{\operatorname{rank}\left(A-B X C^{\star} \pm C X^{\star} B^{\star}\right) \mid X \in \mathbb{F}^{m \times p}\right\} \\
& \quad=\left\{s \mid s \text { is integer and } 2 \mu_{1}+\mu_{4}+\mathcal{K}_{\text {min }} \leq s \leq 2 \mu_{1}+\mu_{4}+\hat{\mathcal{K}}_{\text {max }}\right\} ;
\end{aligned}
$$

- if $\mathbb{F}=\mathbb{R}$ and $A=-A^{T}$, then

$$
\begin{aligned}
& \left\{\operatorname{rank}\left(A-B X C^{T}+C X^{T} B^{T}\right) \mid X \in \mathbb{R}^{m \times p}\right\} \\
= & \left\{s \mid s \text { is even integer and } 2 \mu_{1}+\mu_{4}+\max \{\operatorname{rank}(\mathcal{F}), \operatorname{rank}(\mathcal{G})\}\right. \\
\leq & \left.s \leq 2 \mu_{1}+\mu_{4}+\hat{\mathcal{K}}_{\text {max }}\right\} .
\end{aligned}
$$

Now a simple calculation using Lemmas $9-11$ gives that

$$
2 \mu_{1}+\mu_{4}+\mathcal{K}_{\text {min }}=k_{\text {min }}, \quad 2 \mu_{1}+\mu_{4}+\hat{\mathcal{K}}_{\text {max }}=k_{\text {max }},
$$

and

$$
\begin{aligned}
& 2 \mu_{1}+\mu_{4}+\max \{\operatorname{rank}(\mathcal{F}), \operatorname{rank}(\mathcal{G})\} \\
= & \max \left\{\operatorname{rank}\left[\begin{array}{cc}
A & B \\
B^{T} & 0
\end{array}\right]\right)+2\left(\operatorname{rank}\left[\begin{array}{lll}
A & B & C
\end{array}\right]-\operatorname{rank}\left[\begin{array}{ccc}
A & B & C \\
B^{T} & 0 & 0
\end{array}\right]\right), \\
& \left.\left.\operatorname{rank}\left[\begin{array}{cc}
A & C \\
C^{T} & 0
\end{array}\right]\right)+2\left(\operatorname{rank}\left[\begin{array}{lll}
A & B & C
\end{array}\right]-\operatorname{rank}\left[\begin{array}{ccc}
A & B & C \\
C^{*} & 0 & 0
\end{array}\right]\right)\right\} .
\end{aligned}
$$

Hence, all rank characterizations in Theorem 3 are proved.

[7, Theorem 1.1] characterizes all admissible inertias and the minimal completion rank of the partial matrix of the form

$$
\left[\begin{array}{ccc}
\mathcal{A} & \mathcal{B} & ? \\
\mathcal{B}^{\star} & \mathcal{D} & \mathcal{C} \\
? & \mathcal{C}^{\star} & \mathcal{E}
\end{array}\right]
$$

The following result is a complement to [7, Theorem 1.1], which gives all admissible ranks of the partial matrices.

Theorem 15. Given

$$
\mathcal{A}= \pm \mathcal{A}^{\star} \in \mathbb{F}^{\alpha \times \alpha}, \mathcal{D}= \pm \mathcal{D}^{\star} \in \mathbb{F}^{\beta \times \beta}, \mathcal{E}= \pm \mathcal{E}^{\star} \in \mathbb{F}^{\gamma \times \gamma}, \mathcal{B} \in \mathbb{F}^{\alpha \times \beta}, \mathcal{C} \in \mathbb{F}^{\beta \times \gamma} .
$$

Denote

$$
\begin{aligned}
k_{\min }= & \max \left\{\mathcal{I}_{+}\left(\left[\begin{array}{cc}
\mathcal{D} & \mathcal{C} \\
\pm \mathcal{C}^{\star} & \mathcal{E}
\end{array}\right]\right)+\operatorname{rank}\left[\begin{array}{ccc}
\mathcal{B}^{\star} & \mathcal{D} & \mathcal{C}
\end{array}\right]-\operatorname{rank}\left[\begin{array}{cc}
\mathcal{D} & \mathcal{C}
\end{array}\right],\right. \\
& \left.\mathcal{I}_{+}\left(\left[\begin{array}{cc}
\mathcal{A} & \mathcal{B} \\
\pm \mathcal{B}^{\star} & \mathcal{D}
\end{array}\right]\right)+\operatorname{rank}\left[\begin{array}{ccc}
\mathcal{B}^{\star} & \mathcal{D} & \mathcal{C}
\end{array}\right]-\operatorname{rank}\left[\begin{array}{cc}
\mathcal{B}^{\star} & \mathcal{D}
\end{array}\right]\right\} \\
+ & \max \left\{\mathcal{I}_{-}\left(\left[\begin{array}{cc}
\mathcal{D} & \mathcal{C} \\
\pm \mathcal{C}^{\star} & \mathcal{E}
\end{array}\right]\right)+\operatorname{rank}\left[\begin{array}{lll}
\mathcal{B}^{\star} & \mathcal{D} & \mathcal{C}
\end{array}\right]-\operatorname{rank}\left[\begin{array}{cc}
\mathcal{D} & \mathcal{C}
\end{array}\right],\right. \\
& \left.\mathcal{I}_{-}\left(\left[\begin{array}{cc}
\mathcal{A} & \mathcal{B} \\
\pm \mathcal{B}^{\star} & \mathcal{D}
\end{array}\right]\right)+\operatorname{rank}\left[\begin{array}{lll}
\mathcal{B}^{\star} & \mathcal{D} & \mathcal{C}
\end{array}\right]-\operatorname{rank}\left[\begin{array}{ll}
\mathcal{B}^{\star} & \mathcal{D}
\end{array}\right]\right\},
\end{aligned}
$$

and

$k_{\max }=\min \left\{2 \alpha+\operatorname{rank}\left[\begin{array}{cc}\mathcal{D} & \mathcal{C} \\ \pm \mathcal{C}^{\star} & \mathcal{E}\end{array}\right], 2 \gamma+\operatorname{rank}\left[\begin{array}{cc}\mathcal{A} & \mathcal{B} \\ \pm \mathcal{B}^{\star} & \mathcal{D}\end{array}\right], \alpha+\gamma+\operatorname{rank}\left[\begin{array}{lll}\mathcal{B}^{\star} & \mathcal{D} & \mathcal{C}\end{array}\right]\right\}$

Copyright $@$ by SIAM. Unauthorized reproduction of this article is prohibited. 
- If $\mathbb{F}=\mathbb{C}$, or $\mathbb{F}=\mathbb{R}, \mathcal{A}=\mathcal{A}^{T}, \mathcal{D}=\mathcal{D}^{T}$, and $\mathcal{E}=\mathcal{E}^{T}$, then

$$
\left\{\operatorname{rank}\left[\begin{array}{ccc}
\mathcal{A} & \mathcal{B} & \mathcal{X} \\
\pm \mathcal{B}^{\star} & \mathcal{D} & \mathcal{C} \\
\pm \mathcal{X}^{\star} & \pm \mathcal{C}^{\star} & \mathcal{E}
\end{array}\right] \mid \mathcal{X} \in \mathbb{F}^{\alpha \times \gamma}\right\}=\left\{s \mid s \text { is integer and } k_{\min } \leq s \leq k_{\max }\right\}
$$

- If $\mathbb{F}=\mathbb{R}, \mathcal{A}=-\mathcal{A}^{T}, \mathcal{D}=-\mathcal{D}^{T}$, and $\mathcal{E}=-\mathcal{E}^{T}$, then $k_{\text {min }}$ is reduced to

$$
\begin{aligned}
k_{\min }=\max & \left\{\operatorname{rank}\left[\begin{array}{cc}
\mathcal{D} & \mathcal{C} \\
-\mathcal{C}^{T} & \mathcal{E}
\end{array}\right]+\operatorname{rank}\left[\begin{array}{ccc}
\mathcal{B}^{T} & \mathcal{D} & \mathcal{C}
\end{array}\right]-\operatorname{rank}\left[\begin{array}{cc}
\mathcal{D} & \mathcal{C}
\end{array}\right],\right. \\
& \left.\operatorname{rank}\left[\begin{array}{cc}
\mathcal{A} & \mathcal{B} \\
-\mathcal{B}^{T} & \mathcal{D}
\end{array}\right]+\operatorname{rank}\left[\begin{array}{lll}
\mathcal{B}^{T} & \mathcal{D} & \mathcal{C}
\end{array}\right]-\operatorname{rank}\left[\begin{array}{ll}
\mathcal{B}^{\star} & \mathcal{D}
\end{array}\right]\right\},
\end{aligned}
$$

and

$$
\begin{aligned}
& \left\{\operatorname{rank}\left[\begin{array}{ccc}
\mathcal{A} & \mathcal{B} & \mathcal{X} \\
-\mathcal{B}^{T} & \mathcal{D} & \mathcal{C} \\
-\mathcal{X}^{T} & -\mathcal{C}^{T} & \mathcal{E}
\end{array}\right] \mid \mathcal{X} \in \mathbb{F}^{\alpha \times \gamma}\right\} \\
& \quad=\left\{s \mid s \text { is even integer and } k_{\min } \leq s \leq k_{\max }\right\} .
\end{aligned}
$$

Proof. Theorem 15 is a simple consequence of Theorem 3 with

$$
A=\left[\begin{array}{ccc}
\mathcal{A} & \mathcal{B} & 0 \\
\pm \mathcal{B}^{\star} & \mathcal{D} & \mathcal{C} \\
0 & \pm \mathcal{C}^{\star} & \mathcal{E}
\end{array}\right], \quad B=\left[\begin{array}{c}
I_{\alpha} \\
0 \\
0
\end{array}\right], \quad C=\left[\begin{array}{c}
0 \\
0 \\
I_{\gamma}
\end{array}\right]
$$

Let us finish this section with an example illustrating that none of the inequalities in Theorem 3 are redundant. The example is inspired by a similar example from [7].

Example 3. Let

$$
A=\operatorname{diag}\{1,1,-1,-1,0,0,1,-1,0,0,0,0\}, \quad B=\left[\begin{array}{c}
I_{6} \\
0
\end{array}\right], \quad C=\left[\begin{array}{c}
0 \\
I_{6}
\end{array}\right] .
$$

Then the inequalities in (13) correspond to

$$
2 \leq \mathcal{I}_{+} \leq 7, \quad 2 \leq \mathcal{I}_{-} \leq 7, \quad-3 \leq \mathcal{I}_{+}-\mathcal{I}_{-} \leq 3, \quad \mathcal{I}_{+}+\mathcal{I}_{-} \leq 12 .
$$

5. The nonadjoint/nonsymmetric case. In this section we give the nonadjoint/nonsymmetric version of Theorems 5,1 , and 15 in this section. The following is the nonadjoint/nonsymmetric version of both Theorems 5 and 15 .

TheOREM 16. Let $\mathcal{A} \in \mathbb{F}^{\tilde{n} \times n}, \mathcal{B} \in \mathbb{F}^{\tilde{n} \times m}, \mathcal{C} \in \mathbb{F}^{\tilde{n} \times p}, \mathcal{D} \in \mathbb{F}^{\tilde{m} \times n}, \mathcal{E} \in \mathbb{F}^{\tilde{m} \times p}$, $\mathcal{F} \in \mathbb{F}^{\tilde{p} \times n}$, and $\mathcal{G} \in \mathbb{F}^{\tilde{p} \times m}$. Denote

$$
\begin{gathered}
\mathcal{K}_{\max }=\min \left\{\tilde{m}+\tilde{p}+\operatorname{rank}\left[\begin{array}{ccc}
\mathcal{A} & \mathcal{B} & \mathcal{C}
\end{array}\right], \tilde{m}+p+\operatorname{rank}\left[\begin{array}{cc}
\mathcal{A} & \mathcal{B} \\
\mathcal{F} & \mathcal{G}
\end{array}\right],\right. \\
\left.m+\tilde{p}+\operatorname{rank}\left[\begin{array}{cc}
\mathcal{A} & \mathcal{C} \\
\mathcal{D} & \mathcal{E}
\end{array}\right], m+p+\operatorname{rank}\left[\begin{array}{c}
\mathcal{A} \\
\mathcal{D} \\
\mathcal{F}
\end{array}\right]\right\}
\end{gathered}
$$

and

$$
\begin{aligned}
\mathcal{K}_{\text {min }}= & \operatorname{rank}\left[\begin{array}{lll}
\mathcal{A} & \mathcal{B} & \mathcal{C}
\end{array}\right]+\operatorname{rank}\left[\begin{array}{l}
\mathcal{A} \\
\mathcal{D} \\
\mathcal{F}
\end{array}\right]+\max \left\{\operatorname{rank}\left[\begin{array}{ll}
\mathcal{A} & \mathcal{C} \\
\mathcal{D} & \mathcal{E}
\end{array}\right]\right. \\
& \left.-\operatorname{rank}\left[\begin{array}{ll}
\mathcal{A} & \mathcal{C}
\end{array}\right]-\operatorname{rank}\left[\begin{array}{l}
\mathcal{A} \\
\mathcal{D}
\end{array}\right], \operatorname{rank}\left[\begin{array}{ll}
\mathcal{A} & \mathcal{B} \\
\mathcal{F} & \mathcal{G}
\end{array}\right]-\operatorname{rank}\left[\begin{array}{ll}
\mathcal{A} & \mathcal{B}
\end{array}\right]-\operatorname{rank}\left[\begin{array}{l}
\mathcal{A} \\
\mathcal{F}
\end{array}\right]\right\}
\end{aligned}
$$

Copyright $@$ by SIAM. Unauthorized reproduction of this article is prohibited. 
Then

$\left\{\operatorname{rank}\left[\begin{array}{ccc}\mathcal{A} & \mathcal{B} & \mathcal{C} \\ \mathcal{D} & \mathcal{X} & \mathcal{E} \\ \mathcal{F} & \mathcal{G} & \mathcal{Y}\end{array}\right] \mid \mathcal{X} \in \mathbb{F}^{\tilde{n} \times m}, \mathcal{Y} \in \mathbb{F}^{\tilde{p} \times p}\right\}=\left\{s \mid s\right.$ is integer, $\left.\mathcal{K}_{\min } \leq s \leq \mathcal{K}_{\max }\right\}$.

The proof of Theorem 16 is a direct corollary of results in [8, Theorem 2.2, Lemma 5.3 (see also (5) on page 180) and the observation in the second paragraph of page 166]. Here we include an alternative proof for it to make the paper self-contained.

Proof of Theorem 16. Similar to the proof of Theorem 5, we can find nonsingular matrices $\mathcal{L}_{1}, \tilde{\mathcal{L}}_{1}, \mathcal{L}_{2}, \tilde{\mathcal{L}}_{2}, \mathcal{L}_{3}, \tilde{\mathcal{L}}_{3}$ and two constant matrices $\mathcal{X}_{0}$ and $\mathcal{Y}_{0}$ in $\mathbb{F}$ by using the technique in [7] such that

$$
\begin{aligned}
& \mathcal{L}_{1}\left[\begin{array}{ccc}
\mathcal{A} & \mathcal{B} & \mathcal{C} \\
\mathcal{D} & \mathcal{X} & \mathcal{E} \\
\mathcal{F} & \mathcal{G} & \mathcal{Y}
\end{array}\right] \tilde{\mathcal{L}}_{1} \\
& =\left[\begin{array}{ccccc|cccc|cccc}
I_{n_{1}} & 0 & 0 & 0 & 0 & 0 & 0 & 0 & 0 & 0 & 0 & 0 & 0 \\
0 & 0 & 0 & 0 & 0 & I_{n_{2}} & 0 & 0 & 0 & 0 & I_{n_{2}} & 0 & 0 \\
0 & 0 & 0 & 0 & 0 & 0 & I_{n_{3}} & 0 & 0 & 0 & 0 & 0 & 0 \\
0 & 0 & 0 & 0 & 0 & 0 & 0 & 0 & 0 & I_{n_{4}} & 0 & 0 & 0 \\
0 & 0 & 0 & 0 & 0 & 0 & 0 & 0 & 0 & 0 & 0 & 0 & 0 \\
\hline 0 & I_{n_{5}} & 0 & 0 & 0 & \mathcal{X}_{11} & \mathcal{X}_{12} & \mathcal{X}_{13} & \mathcal{X}_{14} & 0 & 0 & 0 & 0 \\
0 & 0 & I_{n_{6}} & 0 & 0 & \mathcal{X}_{21} & \mathcal{X}_{22} & \mathcal{X}_{23} & \mathcal{X}_{24} & 0 & 0 & 0 & 0 \\
0 & 0 & 0 & 0 & 0 & \mathcal{X}_{31} & \mathcal{X}_{32} & \mathcal{X}_{33} & \mathcal{X}_{34} & 0 & 0 & I_{n_{7}} & 0 \\
0 & 0 & 0 & 0 & 0 & \mathcal{X}_{41} & \mathcal{X}_{42} & \mathcal{X}_{43} & \mathcal{X}_{44} & 0 & 0 & 0 & 0 \\
\hline 0 & 0 & 0 & I_{n_{8}} & 0 & 0 & 0 & 0 & 0 & \mathcal{Y}_{11} & \mathcal{Y}_{12} & \mathcal{Y}_{13} & \mathcal{Y}_{14} \\
0 & I_{n_{5}} & 0 & 0 & 0 & 0 & 0 & 0 & 0 & \mathcal{Y}_{21} & \mathcal{Y}_{22} & \mathcal{Y}_{23} & \mathcal{Y}_{24} \\
0 & 0 & 0 & 0 & 0 & 0 & 0 & I_{n_{9}} & 0 & \mathcal{Y}_{31} & \mathcal{Y}_{32} & \mathcal{Y}_{33} & \mathcal{Y}_{34} \\
0 & 0 & 0 & 0 & 0 & 0 & 0 & 0 & 0 & \mathcal{Y}_{41} & \mathcal{Y}_{42} & \mathcal{Y}_{43} & \mathcal{Y}_{44}
\end{array}\right],
\end{aligned}
$$

where

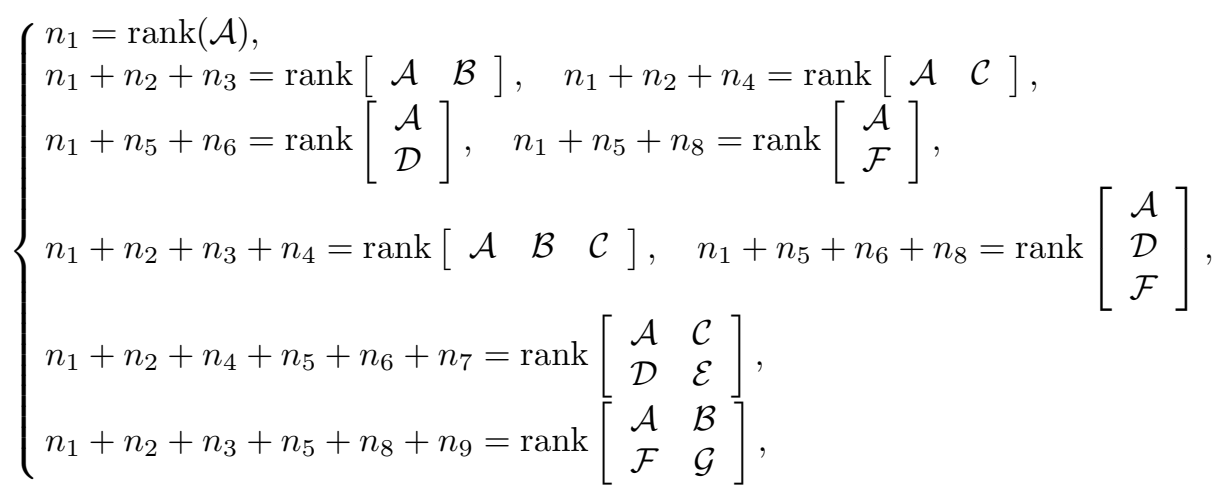

and

$$
\left[\begin{array}{llll}
\mathcal{X}_{11} & \mathcal{X}_{12} & \mathcal{X}_{13} & \mathcal{X}_{14} \\
\mathcal{X}_{21} & \mathcal{X}_{22} & \mathcal{X}_{23} & \mathcal{X}_{24} \\
\mathcal{X}_{31} & \mathcal{X}_{32} & \mathcal{X}_{33} & \mathcal{X}_{34} \\
\mathcal{X}_{41} & \mathcal{X}_{42} & \mathcal{X}_{43} & \mathcal{X}_{44}
\end{array}\right]=\mathcal{L}_{2}\left(\mathcal{X}+\mathcal{X}_{0}\right) \tilde{\mathcal{L}}_{2}, \quad\left[\begin{array}{llll}
\mathcal{Y}_{11} & \mathcal{Y}_{12} & \mathcal{Y}_{13} & \mathcal{Y}_{14} \\
\mathcal{Y}_{21} & \mathcal{Y}_{22} & \mathcal{Y}_{23} & \mathcal{Y}_{24} \\
\mathcal{Y}_{31} & \mathcal{Y}_{32} & \mathcal{Y}_{33} & \mathcal{Y}_{34} \\
\mathcal{Y}_{41} & \mathcal{Y}_{42} & \mathcal{Y}_{43} & \mathcal{Y}_{44}
\end{array}\right]=\mathcal{L}_{3}\left(\mathcal{Y}+\mathcal{Y}_{0}\right) \tilde{\mathcal{L}}_{3}
$$


It is easy to see that

$$
\begin{array}{r}
\operatorname{rank}\left[\begin{array}{ccc}
\mathcal{A} & \mathcal{B} & \mathcal{C} \\
\mathcal{D} & \mathcal{X} & \mathcal{E} \\
\mathcal{F} & \mathcal{G} & \mathcal{Y}
\end{array}\right] \\
=n_{1}+n_{2}+n_{3}+n_{4}+n_{5}+n_{6}+n_{8}+\operatorname{rank}\left[\begin{array}{ccccc}
\mathcal{X}_{44} & -\mathcal{X}_{41} & 0 & 0 & \mathcal{X}_{43} \\
-\mathcal{X}_{14} & \mathcal{Y}_{22}+\mathcal{X}_{11} & \mathcal{Y}_{24} & \mathcal{Y}_{23} & -\mathcal{X}_{13} \\
0 & \mathcal{Y}_{42} & \mathcal{Y}_{44} & \mathcal{Y}_{43} & 0 \\
0 & \mathcal{Y}_{32} & \mathcal{Y}_{34} & \mathcal{Y}_{33} & I_{n_{9}} \\
\mathcal{Y}_{34} & -\mathcal{X}_{31} & 0 & I_{n_{7}} & \mathcal{X}_{33}
\end{array}\right]
\end{array}
$$

Note that

$\mathcal{X}_{44} \in \mathbb{R}^{\left(\tilde{m}-n_{5}-n_{6}-n_{7}\right) \times\left(m-n_{2}-n_{3}-n_{9}\right)}, \mathcal{Y}_{22} \in \mathbb{R}^{n_{5} \times n_{2}}, \mathcal{Y}_{44} \in \mathbb{R}^{\left(\tilde{p}-n_{5}-n_{8}-n_{9}\right) \times\left(p-n_{2}-n_{4}-n_{7}\right)}$, and a tedious calculation gives that

$$
\begin{aligned}
& \max \left\{n_{7}, n_{9}\right\} \\
\leq & \operatorname{rank}\left[\begin{array}{ccccc}
\mathcal{X}_{44} & -\mathcal{X}_{41} & 0 & 0 & \mathcal{X}_{43} \\
-\mathcal{X}_{14} & \mathcal{Y}_{22}+\mathcal{X}_{11} & \mathcal{Y}_{24} & \mathcal{Y}_{23} & -\mathcal{X}_{13} \\
0 & \mathcal{Y}_{42} & \mathcal{Y}_{44} & \mathcal{Y}_{43} & 0 \\
0 & \mathcal{Y}_{32} & \mathcal{Y}_{34} & \mathcal{Y}_{33} & I_{n_{9}} \\
\mathcal{Y}_{34} & -\mathcal{X}_{31} & 0 & I_{n_{7}} & \mathcal{X}_{33}
\end{array}\right] \\
\leq & \min \left\{\tilde{m}+\tilde{p}-n_{5}-n_{3}-n_{8}, \tilde{m}+p+n_{9}-n_{4}-n_{6}, m\right. \\
& \left.+\tilde{p}+n_{7}-n_{3}-n_{8}, m+p-n_{2}-n_{3}-n_{4}\right\} .
\end{aligned}
$$

We have that

$$
s=\operatorname{rank}\left[\begin{array}{ccc}
\mathcal{A} & \mathcal{B} & \mathcal{C} \\
\mathcal{D} & \mathcal{X} & \mathcal{E} \\
\mathcal{F} & \mathcal{G} & \mathcal{Y}
\end{array}\right] \text { for some } \mathcal{X} \in \mathbb{R}^{\tilde{m} \times m} \text { and } \mathcal{Y} \in \mathbb{R}^{\tilde{p} \times p}
$$

if and only if $s$ is an integer satisfying

$$
\begin{aligned}
& \max \left\{n_{7}, n_{9}\right\} \\
\leq & s-\left(n_{1}+n_{2}+n_{3}+n_{4}+n_{5}+n_{6}+n_{8}\right) \\
\leq & \min \left\{\tilde{m}+\tilde{p}-n_{5}-n_{3}-n_{8}, \tilde{m}+p+n_{9}-n_{4}-n_{6},\right. \\
& \left.m+\tilde{p}+n_{7}-n_{3}-n_{8}, m+p-n_{2}-n_{3}-n_{4}\right\} .
\end{aligned}
$$

Thus, Theorem 16 follows directly from (50) and (51).

The following result is the nonadjoint version of Theorem 1 .

TheOREm 17. Let $A \in \mathbb{F}^{\tilde{n} \times n}, B \in \mathbb{F}^{\tilde{n} \times m}, C \in \mathbb{F}^{\tilde{n} \times p}, D \in \mathbb{F}^{\tilde{m} \times n}, F \in \mathbb{F}^{\tilde{p} \times n}$. Denote

$$
\begin{aligned}
k_{\min }= & \operatorname{rank}\left[\begin{array}{ccc}
A & B & C
\end{array}\right]+\operatorname{rank}\left[\begin{array}{c}
A \\
D \\
F
\end{array}\right] \\
& +\max \left\{\operatorname{rank}\left[\begin{array}{cc}
A & C \\
D & 0
\end{array}\right]-\operatorname{rank}\left[\begin{array}{ccc}
A & B & C \\
D & 0 & 0
\end{array}\right]-\operatorname{rank}\left[\begin{array}{cc}
A & C \\
D & 0 \\
F & 0
\end{array}\right],\right. \\
& \left.\operatorname{rank}\left[\begin{array}{cc}
A & B \\
F & 0
\end{array}\right]-\operatorname{rank}\left[\begin{array}{ccc}
A & B & C \\
F & 0 & 0
\end{array}\right]-\operatorname{rank}\left[\begin{array}{cc}
A & B \\
D & 0 \\
F & 0
\end{array}\right]\right\}
\end{aligned}
$$

Copyright $@$ by SIAM. Unauthorized reproduction of this article is prohibited. 
and

$k_{\max }=\min \left\{\operatorname{rank}\left[\begin{array}{lll}A & B & C\end{array}\right], \operatorname{rank}\left[\begin{array}{cc}A & B \\ F & 0\end{array}\right], \operatorname{rank}\left[\begin{array}{cc}A & C \\ D & 0\end{array}\right], \operatorname{rank}\left[\begin{array}{c}A \\ D \\ F\end{array}\right]\right\}$.

Then,

$$
\begin{aligned}
& \left\{\operatorname{rank}(A-B X D-C Y F) \mid X \in \mathbb{F}^{m \times \tilde{m}}, Y \in \mathbb{F}^{p \times \tilde{p}}\right\} \\
& =\left\{s \mid s \text { is integer, } k_{\min } \leq s \leq k_{\max }\right\} .
\end{aligned}
$$

Proof. Theorem 17 follows directly from Theorem 16 and the following equality

$$
\operatorname{rank}(A-B X D-C Y F)=\operatorname{rank}\left[\begin{array}{ccccc}
A & B & C & 0 & 0 \\
D & 0 & 0 & I_{\tilde{m}} & 0 \\
F & 0 & 0 & 0 & I_{\tilde{p}} \\
0 & I_{m} & 0 & -X & 0 \\
0 & 0 & I_{p} & 0 & -Y
\end{array}\right]-(m+\tilde{m}+p+\tilde{p}),
$$

with any $A \in \mathbb{F}^{\tilde{n} \times n}, B \in \mathbb{F}^{\tilde{n} \times m}, C \in \mathbb{F}^{\tilde{n} \times p}, D \in \mathbb{F}^{\tilde{m} \times n}, F \in \mathbb{F}^{\tilde{p} \times n}, X \in \mathbb{F}^{m \times \tilde{m}}$, and $Y \in \mathbb{F}^{p \times \tilde{p}}$.

The particular cases $C=0, F=0$ and $D=I, C=I$ were treated earlier in [19] and [18], respectively.

\section{REFERENCES}

[1] J.K. Baksalary, Nonnegative definite and positive definite solutions to the matrix equation $A X A^{\star}=B$, Linear Multilinear Algebra, 16 (1984), pp. 133-139.

[2] X.-W. Chang And J. WANG, The symmetric solutions of the matrix equations $A X+Y A=C$, $A X A^{T}+B Y B^{T}=C$ and $\left(A^{T} X A, B^{T} X B\right)=(C, D)$, Linear Algebra Appl., 179 (1993), pp. $171-189$.

[3] D. Chu, L. De Lathaumer, and B. De Moor, On the computation of the restricted singular value decomposition via the cosine-sine decomposition, SIAM J. Matrix Anal. Appl., 22 (2000), pp. 580-601.

[4] D. Chu, H.C. Chan, And D.W.C. Ho, Regularization of singular systems by derivative and proportional output feedback, SIAM J. Matrix Anal. Appl., 19 (1998), pp. 21-38.

[5] D. Chu, V. Mehrmann, and N.K. Nichols, Minimum norm regularization of descriptor systems by mixed output feedback, Linear Algebra Appl., 296 (1999), pp. 39-77.

[6] N. Cohen And J. Dancis, Maximal rank Hermitian completions of partially specified Hermitian matrices, Linear Algebra Appl., 244 (1996), pp. 265-276.

[7] N. Cohen And J. Dancis, Inertias of block band matrix completions, SIAM J. Matrix Anal. Appl., 19 (1998), pp. 583-612.

[8] N. Cohen, C.R. Johnson, L. Rodman, And H.J. Woerdeman, Ranks of completions of partial matrices, in The Gohberg Anniversary Collection, Vol. I (Calgary, AB, 1988), Oper. Theory Adv. Appl. 40, Birkhäuser, Basel, 1989, pp. 165-185.

[9] G. Golub and C. Van Loan, Matrix Computations, 3rd ed., The Johns Hopkins University Press, Baltimore, MD, 1996.

[10] J. Gross, Nonnegative-definite and positive-definite solutions to the matrix equation $A X A^{\star}=$ B-revisited, Linear Algebra Appl., 321 (2000), pp. 123-129.

[11] C.G. Khatri And S.K. Mitra, Hermitian and nonnegative definite solutions of linear matrix equations, SIAM J. Appl. Math., 31 (1976), pp. 579-585.

[12] Y. LiU And Y. Tian, More on extremal ranks of the matrix expressions $A-B X \pm X^{\star} B^{\star}$ with statistical applications, Numer. Linear Algebra Appl., 15 (2008), pp. 307-325.

[13] G. Marsaglia and G.P.H. Styan, Equalities and inequalities for ranks of matrices, Linear Multilinear Algebra, 2 (1974), pp. 269-292.

[14] S. Puntanen and G.P.H. Styan, Two matrix-based proofs that the linear estimator Gy is the best linear unbiased estimator, J. Statist. Plan. Inference, 88 (2000), pp. 173-179.

Copyright $@$ by SIAM. Unauthorized reproduction of this article is prohibited. 
[15] H. Qian And Y. Tian, Partially superfluous observations, Econom. Theory, 22 (2006), pp. 529536.

[16] Y. TIAn, The maximal and minimal ranks of some expressions of generalized inverses of matrices, Southeast Asian Bull. Math., 25 (2002), pp. 745-755.

[17] Y. TIAN, Upper and lower bounds for ranks of matrix expressions using generalized inverses, Linear Algebra Appl., 355 (2002), pp. 187-214.

[18] Y. TIAn, The minimal rank of the matrix expression $A-B X-Y C$, Missouri J. Math. Sci., 14 (2002), pp. 40-48.

[19] Y. Tian And S. Cheng, The maximal and minimal ranks of $A-B X C$ with applications, New York J. Math., 9 (2003), pp. 345-362.

[20] Y. TIAn AND D.P. WiEns, On equality and proportionality of ordinary least squares, weighted least squares and best linear unbiased estimators in the general linear model, Statist. Probab. Lett., 76 (2006), pp. 1265-1272.

[21] Y. TIAN AND Y. Liu, Extremal ranks of some symmetric matrix expressions with applications, SIAM J. Matrix Anal. Appl., 28 (2006), pp. 890-905.

[22] Y. Tian And G.P.H. Styan, Cochran's statistical theorem revisited, J. Statist. Plann. Inference, 136 (2006), pp. 2659-2667.

[23] H.J. Woerdeman, Hermitian and normal completions, Linear Multilinear Algebra, 42 (1997), pp. 239-280.

[24] H.J. Woerdeman, Minimal rank completions for block matrices, Linear Algebra Appl., 121 (1989), pp. 105-122.

[25] X. ZHANG, The general common Hermitian nonnegative-definite solution to the matrix equations $A X A^{\star}=B B^{\star}$ and $C X C^{\star}=D D^{\star}$, J. Multivariate Anal., 93 (2005), pp. 257-266.

[26] X. Zhang AND M.Y. Zhang, The rank-constrained Hermitian nonnegative-definite and positive definite solutions to the matrix equation $A X A^{\star}=B$, Linear Algebra Appl., 370 (2003), pp. $163-174$.

Copyright (c) by SIAM. Unauthorized reproduction of this article is prohibited. 